LOCKHEED MARTIN/7

PORTSMOUTH

GASEOUS

DIFFUSION

PLANT
Validation of MCNP4a for Highly Enriched Uranium Using the Battelle Process Safety and Risk Management IBM RS/6000 Workstation \\ by \\ Scott B. Negron \\ Billy L. Lee, Jr. \\ Robert W. Tayloe, Jr.
}




\section{VALIDATION OF MCNP4a FOR HIGHLY ENRICHED URANIUM USING THE BATTELLE PROCESS SAFETY AND RISK MANAGEMENT IBM RS/6000 WORKSTATION}

January 1996

by

Scott B. Negron

Billy L. Lee, Jr.

Robert W. Tayloe, Jr.

Under Contract 400183

to

LOCKHEED MARTIN UTILITY SERVICES, INC.

PORTSMOUTH GASEOUS DIFFUSION PLANT

P.o. Box 628 Piketon, Ohio 45661

Under Contract USECHQ-93-C-0001

to the

\section{U.S. ENRICHMENT CORPORATION}




\section{NOTICE}

This report was prepared as an account of work sponsored by an agency of the United States Government. Neither the United States Government nor any agency thereof, nor any of their employees makes any warranty, express or implied, or assumes any legal liability or responsibility for the accuracy, completeness, or usefulness of any information, apparatus, product, or process disclosed, or represents that its use would not infringe privately owned rights. Reference herein to any specific commercial product, process, or service by trade name, trademark, manufacturer, or otherwise does not necessarily constitute or imply its endorsement, recommendation, or favoring by the United States Government or any agency thereof. The views and opinions of authors expressed herein do not necessarily state or reflect those of the United States Government or any agency thereof.

Available to DOE and DOE Contractors from the Office of Scientific and Technical Information, P.O. Box 62, Oak Ridge, TN 37831; prices available from (615) 576-8401, FTS/626-8401.

Available to the public from the National Technical Information Service, U.S. Department of Commerce, 5285 Port Royal Road, Springfield, VA 22161. 


\section{DISTRIBUTION}

\section{LOCKHEED MARTIN UTILITY SERVICES, INC.}

Portsmouth
J. Harris
R. Dunham
R. Oxenham
R. Lemming
J. Smith
M. Hone

M. Plaster

B. Rumble (4)

Central Files (2)

X-710 Technical Library (2)

Technical Review (2)

Battelle - Columbus

B. Lee (2)

S. Negron

R. Tayloe 


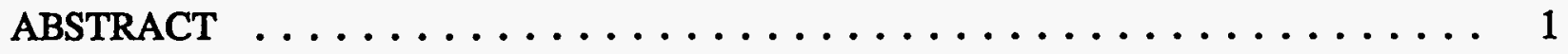

1.0 INTRODUCTION $\ldots \ldots \ldots \ldots \ldots \ldots \ldots \ldots \ldots \ldots \ldots \ldots \ldots \ldots \ldots \ldots$

2.0 CALCULATIONAL PROCEDURE $\ldots \ldots \ldots \ldots \ldots \ldots \ldots \ldots \ldots \ldots$

3.0 DESCRIPTION OF EXPERIMENTS $\ldots \ldots \ldots \ldots \ldots \ldots \ldots \ldots \ldots$

4.0 MCNP INPUT FILES $\ldots \ldots \ldots \ldots \ldots \ldots \ldots \ldots \ldots \ldots \ldots \ldots \ldots \ldots$

5.0 RESULTS $\ldots \ldots \ldots \ldots \ldots \ldots \ldots \ldots \ldots \ldots \ldots \ldots \ldots \ldots$

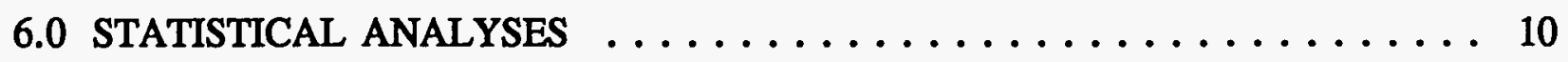

7.0 RANGE OF APPLICABILITY $\ldots \ldots \ldots \ldots \ldots \ldots \ldots \ldots \ldots \ldots$

8.0 CONCLUSIONS $\ldots \ldots \ldots \ldots \ldots \ldots \ldots \ldots \ldots \ldots \ldots \ldots \ldots \ldots \ldots \ldots$

9.0 REFERENCES .............................. 16

APPENDIX A MCNP INPUT FILES $\ldots \ldots \ldots \ldots \ldots \ldots \ldots \ldots \ldots \ldots$

\section{LIST OF TABLES}

Table 1. Validation Data Set Results $\ldots \ldots \ldots \ldots \ldots \ldots \ldots \ldots$ 


\section{ABSTRACT}

This document has been prepared to allow use of the Radiation Shielding \& Information Center (RSIC) release of MCNP4a, which has been installed on the Battelle Process Safety and Risk Management (PSRM) IBM RS/6000 workstation, for production calculations for the Portsmouth Gaseous Diffusion Plant (PORTS). This hardware/software configuration is under the configuration control plan listed in Reference 1.

The first portion of this document outlines basic information with regard to validation of MCNP4a using the supplied cross sections and the additional MCNPDAT cross sections. A basic discussion of MCNP is provided, along with discussions of the validation database in general. A description of the statistical analysis then follows.

The results of this validation indicate that the software and data libraries examined may be used with confidence for criticality calculations at the Portsmouth Gaseous Diffusion Plant (PORTS). When the validation results are treated as a single group, there is a 95 percent confidence that 99.9 percent of future calculations of similar critical systems will have a calculated $k_{\text {eff }}>0.95$. Based on this result, the Battelle PSRM Nuclear Safety Group has adopted the calculational acceptance criteria that a calculated $k_{\text {eff }}+2 \sigma \leq 0.95$ is safely subcritical.

The conclusion of this document is that MCNP4a and all associated cross section libraries installed on the PSRM IBM RS/6000 are acceptable for use in performing production criticality safety calculations for the Portsmouth Gaseous Diffusion Plant. 


\subsection{INTRODUCTION}

The purpose of this validation is to provide reasonable assurance that the Battelle Process Safety and Risk Management IBM RS/6000 workstation implementation of MCNP4a and all associated cross section libraries are valid for use in the analysis and subsequent evaluation of various plant site activities involving enriched uranium at the Portsmouth Gaseous Diffusion Plant (PORTS).

This validation effort has been patterned after a previous validation study documented in POEF-SH-23 for the Battelle PSRM IBM RS/6000 implementation of the CSAS25 calculational sequence in SCALE-4.2 and the 27 energy group ENDF/B-IV cross section library (Ref. 2). All calculations were performed on the Battelle PSRM IBM RS/6000 workstation. This validation covers the MCNP4a code, the supplied cross-section data set, and the additional MCNPDAT cross section library.

A total of 127 cases, consisting of highly enriched systems ( $>90 \%{ }^{235} \mathrm{U}$ ) were run. These cases consisted of various $\mathrm{UO}_{2} \mathrm{~F}_{2}$ and $\mathrm{UO}_{2}\left(\mathrm{NO}_{3}\right)_{2}$ solution systems, oxide-water systems, and several miscellaneous combinations of uranium and moderating materials. All of the executed cases have applicability to operations at PORTS.

The data obtained from the calculations were tabulated and analyzed to determine normality. Based on the statistical analyses, the lower limit for $\mathrm{k}_{\text {eff }}$ with 95 percent confidence and a 99.9 percent one-sided tolerance is 0.9667 . Therefore, for the anticipated range of materials to be encountered at PORTS, the version of MCNP4a (and all cross section libraries used in the validation runs) installed on the Battelle PSRM IBM RS/6000 workstation is safe for production use at PORTS provided $k_{\text {eff }}+2 \sigma$ does not exceed a value of 0.95 . The margin between 0.95 and 0.9667 has been adopted as an additional safety margin and to allow for consistence with the value of 0.95 that has been traditionally used. 


\subsection{CALCULATIONAL PROCEDURE}

A complete description of the MCNP4a code package can be found in the RSICsupplied user manual (Ref. 3). MCNP is a monte carlo neutron and photon transport code which uses combinatorial geometry for the construction of three dimensional models. Mnemonic symbols are used to represent two dimensional geometric surfaces (surface cards), which are combined in the cell cards to form three dimensional regions called cells. The cell cards also contain the material number associated with a mixture of isotopes specified at the end of the input deck.

Cells may be combined in many ways to form complex geometric models. All points outside of the model are located in the universe. Each cell (and the universe) must be assigned an importance, which "weights" all particles entering or leaving the cell. The user must also specify the number of particle histories to be run. For criticality $\left(\mathrm{k}_{\mathrm{eff}}\right)$ calculations starting "source" particles, which initiate all fission neutrons, must also be assigned.

The MCNP executable code reads in the input deck and then begins the problem. Cross sections corresponding to the isotopes specified in the material cards are accessed through a directory (XSLIB), which specifies the file and location within the file for each isotope listed. 


\subsection{DESCRIPTION OF EXPERIMENTS}

Complete descriptions of all experiments can be found in References 4-10. Experiments reported in Reference 4 can be broken up into three general groups:

1) Single unreflected cylinders

2) Single cylinders reflected from all six sides

3) Arrays of cylinders reflected from all six sides

The single cylinders were made of either aluminum, stainless steel, or aluminum with a stainless steel sleeve. Cylinders in arrays were all aluminum, although some experiments used stainless steel sleeves. The reflected experiments were performed inside a cube of reflecting material with an inside dimension of $\approx 122 \mathrm{~cm}$. The reflecting material was either $\approx 27.7 \mathrm{~cm}$ of concrete or $\approx 20.6 \mathrm{~cm}$ of plexiglass. Uranium concentration varied between $\approx 55 \mathrm{gU} / 1$ and $\approx 360 \mathrm{gU} / 1$. The fissile solutions were all uranium nitrate.

Experiments included from Reference 5 were either single spheres or single cylinders, all unreflected. The uranyl nitrate solutions had uranium concentrations that varied between $\approx 14 \mathrm{gU} / 1$ and $\approx 28 \mathrm{gU} / 1$. Some experiments used boron as a soluble poison. The spherical container was made of aluminum while the cylindrical ones were carbon steel.

Reference 6 experiments were all 3-D arrays of identical elements. Each element was a $19.04 \mathrm{~cm}$ i.d. plexiglass cylinder filled with 5 liters of uranyl nitrate. The uranyl nitrate had a uranium concentration of $415 \mathrm{gU} / \mathrm{l}$. The entire array was either unreflected or reflected on six sides with paraffin of various thicknesses.

Experiments from Reference 7 used parallelepipeds of uranyl fluoride to achieve criticality. Single units as well as two units side by side were considered. A tight fitting 
water reflector existed in some instances and a uranium concentration of $94.18 \mathrm{gU} / 1$ was used throughout.

Reference 8 experiments consisted of single spheres of uranyl fluoride, one unreflected and the remainder water reflected. Uranium concentration varied between $\approx 20$ and $\approx 213$ $\mathrm{gU} / 1$.

Experiments reported in Reference 9 used cylinders of uranyl fluoride. These experiments are to be distinguished from the others included in this report in that the uranium concentration was extremely high, either 889.46 or $576.95 \mathrm{gU} / \mathrm{l}$. All had a tight fitting water reflector.

Experiments from Reference 10 consisted of arrays of cans of uranium dioxide. Polyethylene, plexiglass and alcohol were used as reflectors, external moderators, and internal moderators, respectively. The uranium was $93.15 \%$ enriched. The $\mathrm{UO}_{2}$ mass per container was either $0.421 \mathrm{~kg}, 17 \mathrm{~kg}$, or $20 \mathrm{~kg}$. 


\subsection{MCNP INPUT FILES}

A total of 127 individual input files were run for this validation. These files were obtained through the courtesies of C. Crawford and B. Palmer of the Idaho National Engineering Laboratory (INEL). These files were transmitted electronically from INEL to the Battelle PSRM IBM RS/6000. These files are the same ones used for the INEL validation of MCNP documented in Reference 11.

A few minor changes were made in these files to enable them to run on the Battelle IBM. These changes include changing the isotope ZAID for several isotopes used in the INEL files which have been superseded in the latest version of MCNP. An example of this is $25055.50 \mathrm{c}$, the superseded ZAID for manganese 55, being replaced by 25055.51c. Other than superseded ZAIDs, the only other changes were editing a few files to get them all in an 80 column format. No changes were made that in any way effected the accuracy of the models. Although no direct comparison of the results of the Battelle data was made to the INEL data, a cursory comparison shows excellent agreement. 


\subsection{RESULTS}

The resulting eigenvalues obtained from the 127 cases run are listed in Table 1, along with the standard deviation for each input file. The high and low values of $k_{\text {eff }}$ are 1.032 and 0.953 , respectively. The average value of $k_{e f f}$ is 1.004 . The corresponding Reference number is listed in parenthesis next to each filename. This reference gives detailed information regarding the corresponding critical system.

Table 1. Validation Data Set Results

\begin{tabular}{|c|c|c|c|c|c|}
\hline $\begin{array}{l}\text { FILENAME } \\
\text { (Reference) }\end{array}$ & . & SIGMA & $\begin{array}{l}\text { EIIGEAME } \\
\text { (Reference) }\end{array}$ & kert & SIGMA \\
\hline GWI01 (5) & 0.99839 & 0.00199 & FOX $05(8)$ & 1.00272 & 0.00174 \\
\hline GWI02 (5) & 0.99106 & 0.00192 & FOX $06(8)$ & 1.00137 & 0.00293 \\
\hline GWI03 (5) & 0.98591 & 0.00185 & FOX 07 (8) & 0.99702 & 0.00290 \\
\hline GWI04 (5) & 0.98779 & 0.00228 & FOX 08 (8) & 1.00040 & 0.00168 \\
\hline GWI05 (5) & 1.00527 & 0.00154 & FOX 09 (9) & 0.99909 & 0.00336 \\
\hline GWI06 (5) & 1.00904 & 0.00170 & FOX 10 (9) & 1.00359 & 0.00328 \\
\hline GWI07 (5) & 1.00322 & 0.00139 & FOX 11 (9) & 0.97306 & 0.00300 \\
\hline GW108 (5) & 1.00451 & 0.00127 & FOX $12(9)$ & 0.95354 & 0.00283 \\
\hline GWI09 (5) & 0.99559 & 0.00115 & ROT 01 (4) & 1.00411 & 0.00354 \\
\hline MAG01 (10) & 1.03188 & 0.00314 & ROT 02 (4) & 1.01065 & 0.00341 \\
\hline MAG02 (10) & 1.00505 & 0.00267 & ROT 03 (4) & 1.01581 & 0.00318 \\
\hline MAG03 (10) & 0.99835 & 0.00252 & ROT 04 (4) & 1.00786 & 0.00360 \\
\hline MAG04 (10) & 0.99873 & 0.00252 & ROT 05 (4) & 1.00054 & 0.00370 \\
\hline MAG05 (10) & 0.99032 & 0.00242 & ROT 06 (4) & 1.00181 & 0.00340 \\
\hline MAG06 (10) & 1.01180 & 0.00276 & ROT 07 (4) & 1.00253 & 0.00355 \\
\hline MAG07 (10) & 0.99577 & 0.00280 & ROT 08 (4) & 1.00834 & 0.00319 \\
\hline MAG08 (10) & 1.01570 & 0.00282 & ROT 09 (4) & 1.00028 & 0.00325 \\
\hline MAG09 (10) & 0.99733 & 0.00282 & ROT $11(4)$ & 1.01814 & 0.00375 \\
\hline MAG10 (10) & 1.01058 & 0.00285 & ROT 12 (4) & 1.00868 & 0.00329 \\
\hline MAG11 (10) & 1.00252 & 0.00271 & ROT $13(4)$ & 1.01078 & 0.00353 \\
\hline MAG12 (10) & 0.99917 & 0.00269 & ROT $14(4)$ & 1.00460 & 0.00371 \\
\hline MAG13 (10) & 0.99107 & 0.00244 & ROT 15 (4) & 1.01070 & 0.00337 \\
\hline
\end{tabular}




\begin{tabular}{|c|c|c|c|c|c|}
\hline $\begin{array}{l}\text { FLLNAMAE } \\
\text { Reference) }\end{array}$ & $=k_{f r}$ & SIGMA & $\begin{array}{l}\text { FIFENAME } \\
\text { (Reference) }\end{array}$ & $\mathrm{H}+\mathbf{L}_{\mathrm{eft}}$ & SIGMA \\
\hline MAG14 (10) & 1.02213 & 0.00278 & ROT $16(4)$ & 1.00400 & 0.00358 \\
\hline MAG15 (10) & 1.00530 & 0.00242 & ROT 17 (4) & 1.00919 & 0.00366 \\
\hline MAG16 (10) & 0.99589 & 0.00294 & ROT 18 (4) & 1.00505 & 0.00349 \\
\hline MAG17 (10) & 0.99488 & 0.00283 & ROT $19(4)$ & 1.00449 & 0.00339 \\
\hline MAG18 (10) & 0.99905 & 0.00277 & ROT 20 (4) & 1.00654 & 0.00312 \\
\hline MAG19 (10) & 0.99504 & 0.00250 & ROT 21 (4) & 1.00316 & 0.00369 \\
\hline MAG20 (10) & 1.00048 & 0.00280 & ROT $22(4)$ & 1.01086 & 0.00371 \\
\hline FOX01 (7) & 0.99935 & 0.00307 & ROT 23 (4) & 1.01203 & 0.00331 \\
\hline FOX02 (7) & 1.00190 & 0.00320 & ROT 24 (4) & 1.00803 & 0.00326 \\
\hline FOX03 (7) & 0.99300 & 0.00362 & ROT 25 (4) & 1.01092 & 0.00350 \\
\hline FOX04 (7) & 1.00567 & 0.00322 & ROT 26 (4) & 0.99947 & 0.00366 \\
\hline ROT27 (4) & 1.00228 & 0.00344 & ROT 64 (4) & 1.00167 & 0.00348 \\
\hline ROT28 (4) & 1.00636 & 0.00329 & ROT 65 (4) & 1.01319 & 0.00313 \\
\hline ROT29 (4) & 0.99491 & 0.00352 & ROT 66 (4) & 1.01411 & 0.00342 \\
\hline ROT30 (4) & 1.01064 & 0.00367 & ROT 67 (4) & 1.00702 & 0.00293 \\
\hline ROT31 (4) & 1.00103 & 0.00309 & ROT 68 (4) & 0.99842 & 0.00293 \\
\hline ROT32 (4) & 1.00601 & 0.00269 & ROT 69 (4) & 1.00062 & 0.00282 \\
\hline ROT33 (4) & 1.00735 & 0.00306 & ROT 70 (4) & 0.99956 & 0.00277 \\
\hline ROT34 (4) & 1.00447 & 0.00336 & ROT $71(4)$ & 1.00396 & 0.00266 \\
\hline ROT35 (4) & 1.00329 & 0.00286 & ROT $72(4)$ & 1.00410 & 0.00276 \\
\hline ROT36 (4) & 1.00782 & 0.00292 & ROT 73 (4) & 1.01062 & 0.00291 \\
\hline ROT37 (4) & 0.99906 & 0.00328 & ROT 74 (4) & 1.00761 & 0.00358 \\
\hline ROT38 (4) & 1.02456 & 0.00333 & ROT $75(4)$ & 1.00577 & 0.00334 \\
\hline ROT39 (4) & 1.00767 & 0.00269 & ROT $76(4)$ & 1.00978 & 0.00314 \\
\hline ROT40 (4) & 1.00626 & 0.00295 & ROT 77 (4) & 1.00421 & 0.00280 \\
\hline ROT41 (4) & 1.00862 & 0.00309 & ROT 78 (4) & 1.00470 & 0.00343 \\
\hline ROT42 (4) & 0.99989 & 0.00353 & ROT 79 (4) & 1.00400 & 0.00299 \\
\hline ROT43 (4) & 0.99437 & 0.00315 & ROT 80 (4) & 1.00627 & 0.00314 \\
\hline ROT50 (4) & 1.01107 & 0.00294 & THO $01(6)$ & 0.99748 & 0.00379 \\
\hline ROT51 (4) & 1.01231 & 0.00321 & THO $02(6)$ & 1.00382 & 0.00351 \\
\hline ROT52 (4) & 1.01374 & 0.00319 & THO $03(6)$ & 1.02282 & 0.00354 \\
\hline ROT53 (4) & 1.00622 & 0.00251 & THO 04 (6) & 1.03022 & 0.00329 \\
\hline ROT54 (4) & 1.00556 & 0.00249 & THO 05 (6) & 1.02464 & 0.00313 \\
\hline ROT55 (4) & 1.00396 & 0.00343 & THO 06 (6) & 0.99538 & 0.00345 \\
\hline
\end{tabular}




\begin{tabular}{|c|c|c|c|c|c|}
\hline $\begin{array}{l}\text { FILENAME } \\
\text { (Reference) }\end{array}$ & $2 \mathrm{l}_{\mathrm{rf}}$ & SIGMA & $\begin{array}{l}\text { FIGENAME } \\
\text { (Reference) }\end{array}$ & 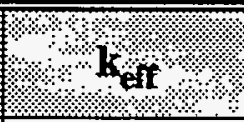 & SIGMA \\
\hline ROT56 (4) & 1.00687 & 0.00311 & THO 07 (6) & 0.98434 & 0.00323 \\
\hline ROT57 (4) & 1.01244 & 0.00327 & THO 08 (6) & 1.00595 & 0.00321 \\
\hline ROT58 (4) & 1.00848 & 0.00264 & THO 09(6) & 1.02781 & 0.00301 \\
\hline ROT59 (4) & 1.00072 & 0.00348 & THO $10(6)$ & 1.03251 & 0.00281 \\
\hline ROT60 (4) & 0.99534 & 0.00350 & THO $11(6)$ & 0.99362 & 0.00336 \\
\hline ROT61 (4) & 1.00497 & 0.00292 & THO $12(6)$ & 0.97328 & 0.00370 \\
\hline ROT62 (4) & 1.00337 & 0.00310 & THO $13(6)$ & 0.97435 & 0.00315 \\
\hline ROT63 (4) & 1.00371 & 0.00369 & & & \\
\hline
\end{tabular}




\subsection{STATISTICAL ANALYSES}

A cursory review of the data revealed that it appears to approximate a normal distribution. If the data approximate a normal distribution, then standard tabulated one-sided tolerance factors for normal distributions may be applied to calculate the minimum acceptable $\mathrm{k}_{\text {eff }}$ for the data, $\mathrm{k}_{\min }$.

The first step performed on the data was a calculation of the mean, median, and mode. In a perfectly normal distribution, these three values are equivalent. The mean may be calculated from

$$
\bar{x}=\frac{1}{N} \sum_{i=1}^{N} x_{i}
$$

where $\mathbf{N} \equiv$ number of data points.

The median is that value where an equivalent number of values fall both above and below it. In the case of ungrouped data, sorted in ascending order, the value of the median may be taken to be the value of the $(N+1) / 2$ th item for odd $N$; for even $N$, it is customarily taken as the arithmetic average of the (N/2)th item and the $(N / 2+1)$ th item.

The mode is defined as that value in a distribution about which the values are most heavily concentrated, and may be calculated by the empirical relation:

$$
\text { Mode }=\bar{x}-\sigma\left[\frac{\sqrt{\beta_{1}}\left(\beta_{2}+3\right)}{2\left(5 \beta_{2}-6 \beta_{1}-9\right)}\right]
$$

where:

$$
\begin{aligned}
& \sigma=\text { standard deviation about the mean } \\
& \sqrt{ } \beta_{1}=\text { coefficient of skewness } \\
& \beta_{2}=\text { coefficient of kurtosis }
\end{aligned}
$$


The mean was then calculated as

$$
\bar{x}=1.00146
$$

with

$$
\sigma=0.0107
$$

The skewness and kurtosis coefficients were respectively

$$
\checkmark \beta_{1}=-0.7915 \quad \text { and } \quad \beta_{2}=4.736
$$

The mean was 1.004 , the median 1.004 , and the mode, calculated from the above equation, was 1.004 .

The mean, median, and mode are all identical to 4 significant figures, which is a good indication of normally distributed data.

The skewness coefficient, a measure of the degree of symmetry in a distribution, for a perfectly normal distribution (i.e., or one perfectly symmetric about the mean) would be 0.0 . The kurtosis coefficient, a measure of the peakedness in a distribution, for a perfectly normal distribution would be 3.0. The values obtained for this analysis $(-0.791$ and 4.736$)$ for the skewness and kurtosis coefficients, respectively, are sufficiently close to those expected from a perfectly normal distribution; therefore, it can be considered normally distributed data.

The one-sided tolerance limit for a normal distribution was used to determine the $\mathrm{k}_{\min }$ to be applied to the system. For a one-sided tolerance limit for a normal distribution, criticality safety personnel are only interested in estimating a value above which a proportion will lie to a certain confidence level. The value of $k_{\min }$ is then established from

\section{Final Report}

January 1996 


$$
k_{\min }=\bar{k}-K^{*} \sigma
$$

where $K^{*}$ is the tolerance factor. For $95 \%$ confidence that $99.9 \%$ of the $\mathrm{k}_{\text {eff }}$ values calculated in future calculations will fall above $k_{\min }$ is $K^{*}=3.4836$

$$
\begin{gathered}
\mathrm{k}_{\min }=1.004-3.4836(0.0107) \\
\mathrm{k}_{\min }=0.9667
\end{gathered}
$$

As stated earlier, to maintain consistency with past operations, $\mathrm{k}_{\min }$ will continue to be assumed to be 0.95 , with the difference between 0.95 and the calculated $k_{\min }$ being added to the margin of subcriticality.

Bias is defined as the deviation of the calculated values of $k_{\text {eff }}$ from unity, or an average bias which is taking the difference between an average of the calculated results and unity. For this validation effort, the bias has been established as

$$
k_{b}=|1-\bar{k}|
$$

which gives $k_{b}=0.0038$.

The margin of subcriticality $\left(k_{m}\right)$ can then be calculated based on the following relationship.

$$
\mathrm{k}_{\min } \leq 1-\mathrm{k}_{\mathrm{b}}-1.645 \mathrm{k}_{\sigma}-\mathrm{k}_{\mathrm{m}}
$$

Which is rearranged to give:

$$
\mathrm{k}_{\mathrm{m}} \leq 1-\mathrm{k}_{\mathrm{b}}-1.645 \mathrm{k}_{\sigma}-\mathrm{k}_{\min }
$$


The incorporation of the 1.645 multiplier on $\mathbf{k}_{\sigma}$ is necessary to place the calculation at the one-sided 95\% confidence level. Substituting this gives:

$$
k_{m} \leq 1-0.004-1.645(0.0107)-0.95=0.0284
$$

The margin of subcriticality for calculations based on the validation is less than or equal to 0.0284 . 


\subsection{RANGE OF APPLICABILITY}

It is considered that this validation covers a vast majority of the various fissile systems found at PORTS, and that it is appropriate to use MCNP4a and all cross sections used in the validation cases to analyze them. Section 3 of this report outlines the range of critical experiments covered in this validation. All types of fuels and moderators, reflectors, and arrays that might be used to evaluate systems at PORTS are exercised in this validation data set. Only high enriched ( $>90 \%{ }^{235} \mathrm{U}$ ) cases were run in this validation. Therefore, only calculations in which the uranium enrichment is greater than or equal to 90 percent can be considered valid for use in criticality safety analysis. All aspects of MCNP's $k_{\text {eff }}$ calculational procedures are exercised by the validation data set as well. It is therefore considered that the MCNP package on the Battelle IBM is applicable to criticality evaluation of highly enriched systems which could be found at PORTS. 


\subsection{CONCLUSIONS}

The results of this validation indicate that the PSRM IBM RS/6000 version of MCNP4a may be used with confidence for criticality calculations of high enriched uranium systems at the Portsmouth Gaseous Diffusion Plant. When the validation results are treated as a single group, there is a $95 \%$ confidence that $99.9 \%$ of future calculations of similar critical systems will have a calculated $k_{\text {eff }}>0.9667$. Based on this result, the Portsmouth Nuclear Criticality Safety Department has adopted the calculational acceptance criteria that a $\mathrm{k}_{\text {eff }}+2 \sigma \leq 0.95$ is safely subcritical. 


\subsection{REFERENCES}

1. "Nuclear Safety Software System Catalog (IBM RS/6000)," Battelle Process Safety and Risk Management, PSRM-NS-01, Rev. 1, September 1994.

2. "Validation of the CSAS25 Calculational Sequence in SCALE-4.2 and the 27 Energy Group ENDF/B-IV Cross Sections on the Battelle Process Safety and Risk Management IBM RS/6000 Workstation," Battelle, December 1994.

3. Briesmeister, J. (Editor), "MCNP-A General Monte Carlo Code for Neutron and Photon Transport, Version 3A, LA-7396-M, Rev. 2," September 1986, revised April 1991.

4. R.E. Rothe and I Oh, "Benchmark Critical Experiments on High Enriched Uranyl Nitrate Solution Systems," NUREG/CR-0041, Rockwell International, April 1978

5. R. Gwin and D.W. Magnuson, "The Measurement of Eta and Other Nuclear Properties of U-233 and U-235 in Critical Aqueous Solutions," Nuclear Science and Engineering 12, 364-380, 1962.

6. J.T. Thomas, "Critical Three Dimensional Arrays of Neutron Interacting Units," ORNL-TM-719, Oak Ridge National Laboratory, October 1963.

7. J.K. Fox, "Some Studies of Water, Styrofoam, and Plexiglass Reflectors," ORNL2609, Oak Ridge National Laboratory, February 1958.

8. J.K. Fox, "Critical Parameters of Uranium Solutions in Simple Geometry," ORNL2609, Oak Ridge National Laboratory, February 1958. 
9. J.K. Fox et al, "Critical Mass Studies, Part IX, Aqueous U-235 Solutions, " ORNL2367, Oak Ridge National Laboratory, March 1958.

10. D.W. Magnuson, "Critical Dimensions with Enriched Uranium Dioxide," Y-DR-120, Oak Ridge Y-12 Plant, November 1973.

11. C. Crawford and B.M. Palmer, "Validation of MCNP, A Comparison with SCALE," Parts 1\&3, Idaho National Engineering Laboratory, WINCO-1111, October 1992. 
POEF-SH-33

APPENDIX A

\section{MCNP INPUT FILES}




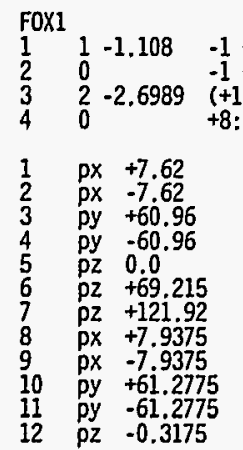

\section{mode $n$}

imp:n 11110

$\begin{array}{ll}92234.50 \mathrm{c} & 2.52028-6 \\ 92235.50 \mathrm{c} & 2.24845-4\end{array}$

$92236.50 \mathrm{c} \quad \mathbf{6} .48749-7$

$\begin{array}{rr}92236.50 \mathrm{C} & \mathbf{6} .48749-7 \\ 8016.50 \mathrm{C} & 3.34432-2\end{array}$

$8016.50 \mathrm{C} \quad 3.34432-2$

$\begin{array}{ll}9019.50 \mathrm{C} & 4.82283-4 \\ 1001.50 \mathrm{C} & 6.59219-2\end{array}$

mt1 lwtr, $01 \mathrm{t}$

kcode $10001.0 \quad 10100$

ksrc 005

print

$\begin{array}{lll}\text { FOX2 } & \\ 1 & 1 & -1.108 \\ 2 & 0 & \\ 3 & 2 & -2.6989 \\ 4 & 3 & -.9982 \\ 5 & 0 & \\ 6 & 0 & \end{array}$

$-1+2-3+4+5-6$

$-1+2-3+4+6-7$

+1: $-2:+3:-4:-5)-8+9-10+11-7+12$

SCUBOID OF URANYL FLUORIDE

SCUBOID VOID

SCUBOTD OF ALUMINUM

\$ZERO IMPORTANCE OUTSIDE WORLD

$p x+7.62$

(+8:-9:+10:-11:-12) $-13+14-15+16-6+17$

$+13:-14:+15:-16:+7:-17$

SCUBOID OF URANYL FLUORIDE

SCUBOIO OF ALUMINUM

SWATER REFLECTOR

SVOID

SZERO IMPORTANCE OUTSIDE WORLD

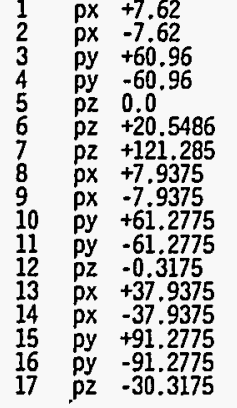

mode $n$

imp: n 1 4r 0

$\mathrm{ml}$. $92234.50 \mathrm{c}$

$92235.50 c$

$92238.50 \mathrm{c}$

$92236,50 \mathrm{c}$

(8016.50

mt1 iwtr.01t

$\begin{array}{lrr}\mathrm{m} 2 & 13027.50 \mathrm{c} & \frac{1}{2} \\ \mathrm{~m} 3 & 1001.50 \mathrm{c} & 2\end{array}$

mt3 iwtr.01t

kcode 10001.010100

ksrc 005

print

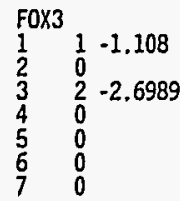

$-1+2-3+4+5-6 \quad u=1$

$\begin{array}{lll}-1+2-3+4+6-7 & u=1 \\ (-1:-2:+3:-4:-5) & u=1 \\ u=1 & \end{array}$

$-13+14-15+16-17+18$

thil $\operatorname{trcl}=(-15.557500)$

$-19+20-21+22-23+24=4$ t $\# 5$ trcl $=(+15.557500)$

$+19:-20:+21 ;-22:+23:-24$

SCUBOID OF URANYL FLUORIDE

CUBOID VOID

SUBOID OF ALUMINUM

CELL FILLED WITH 1-3

SLIKE 4

SVOID

SZERO IMPORTANCE OUTSIDE WORLD

Final Report

January 1996

Validation of MCNP4A on the Battelle

PSRM IBM RS/6000 Workstation

Page 19 


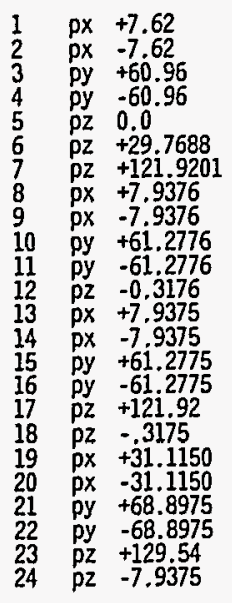

mode $n$

imp: $125 r \quad 0$

$92234.50 \mathrm{c} \quad 2.52028-6$

$1.31278-5$

$92236.50 \mathrm{c} \quad 6.48749-7$

$9019.50 \mathrm{C}$

3. $34432-2$

4.82283-4

mt1 lwtr.01t

6.59219-2

$\mathrm{m2} 13027.50 \mathrm{c}, 1$

ksrc 15,5575 o 5 - -15.557505

print

$\begin{array}{llll}\text { FOX44 } & & \\ 1 & 1 & -1.108 & -1 \\ 2 & 0 & & -1 \\ 3 & 2 & -2.6989 & (+1 \\ 4 & 0 & & -13 \\ 5 & 0 & & -13 \\ 6 & 3 & -0.9982 & -19 \\ 7 & 0 & & -19 \\ 8 & 0 & & +19 \\ & & & \\ 1 & p x & +7.62 \\ 2 & p x & -7.62 \\ 3 & p y & +60.96 \\ 4 & p y & -60.96 \\ 5 & p z & 0.0 \\ 6 & p z & +20.056 \\ 7 & p z & +121.2851 \\ 8 & p x & +7.9376 \\ 9 & p x & -7.9376 \\ 10 & p y & +61.2776 \\ 11 & p y & -61.2776 \\ 12 & p z & -0.3176 \\ 13 & p x & +7.9375 \\ 14 & p x & -7.9375 \\ 15 & p y & +61.2775 \\ 16 & p y & -61.2775 \\ 17 & p z & +121.285 \\ 18 & p z & -0.3175 \\ 19 & p x & +53.4950 \\ 20 & p x & -53.4950 \\ 21 & p y & +91.2775 \\ 22 & p y & -91.2775 \\ 23 & p z & -30.3175\end{array}$

SCUBOID OF URANYL FLUORIDE

SCUBOID VOID

$(+1:-2:+3:-4:-5:+7)-8+9-10+11-7+12$ u=1
$-13+14-15+16-17+18$ fi $1=1$ trc $1=(-15.5575$ SCUBOID OF ALUMINUM
SCELL FILL WITH $1-3$

$+2-3+4+5-6 \quad u=1$
$+2-3+4+6-7 \quad u=1$

$-13+14-15+16-17+18$ fijl=1 trCl $=(+15.557500)$ SLIKE 4

SWATER REFLECTOR

$-19+20-21+22-6+23 \# 4 \quad \# 5$

$+19 \cdot-20=+21=-22 \cdot+17=-23$

SZERO IMPORTANCE OUTSIDE WORLD

mode $n$

imp: $n 16 r 0$

mi $192234.50 \mathrm{c}$

$92234.50 \mathrm{c}$

$92238.50 \mathrm{c}$

$92236.50 \mathrm{c}$

$8016.50 \mathrm{C}$

$9019.50 \mathrm{c}$

$1001.50 \mathrm{C}$

mt1 lwtr.01t

4.82283-4

$\mathrm{m} 2 \quad 13027.50 \mathrm{c}$

$3016.50 \mathrm{c}$

mt3 iwtr.oit

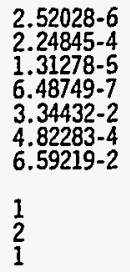

\section{Final Report}

January 1996 
kcode 10001.010100

$\begin{array}{lllllllllll} & k \text { ksrc } & -15.5575 & 0 & 10 & 15.5575 & 0 & 10\end{array}$

print 
kcode 10001.010100

$\begin{array}{lllllll}\text { ksrc } & -15.5575 & 0 & 10 & 15.5575 & 0 & 10\end{array}$

print 
FOX5

$\begin{array}{llll}\text { FOX5 } & & & \\ \frac{1}{2} & 1 & -1.02 & -1 \\ 3 & 2 & -2.6989 & +1 \\ 3 & 0 & & +2\end{array}$

$\begin{array}{lll}1 & \text { so } & 34.6 \\ 2 & \text { so } & 34.92\end{array}$

mode $n$

imp:n 110

$\min _{9}: n_{92234,50 c}$ 9.77288-5 2.78669-6

$1.37713-7$

50

lwtr.01t

$3.33396-2$

$1.02376-4$
$6.64745-2$

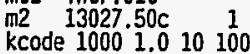

ksrc 0000

print

\section{F0X6 \\ $\begin{array}{llll}1 & -1.4 & -1 & \\ 2 & -2.6989 & +1 & -2 \\ 3 & -0.9982 & +2 & -3 \\ 0 & & +3\end{array}$ \\ $\begin{array}{lll}1 & 50 & 11.51765 \\ 2 & 50 & 11.67765 \\ 3 & 50 & 41.67765\end{array}$}

mode $n$

imp: $n 1110$

92234.50 $\quad 9.32864-6$

2235.50c $8.32245-4$

92238.50c $4.85914-5$

$2.40129-6$

$8016.50 \mathrm{c} \quad 3.34713-2$

$\begin{array}{ll}9019.50 c & 1.78513-3 \\ 1001.50 c & 6.33723-2\end{array}$

mt1 liwtr olt

$\begin{array}{lrl}\mathrm{m} 2 & 13027.50 \mathrm{C} & 1 \\ \mathrm{~m} 3 & 1001.50 \mathrm{C} & 2\end{array}$

mt3 lwtr oit

kcode 10001.010100

$k$ code 1000

$\mathrm{ksrc}$
print

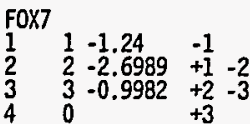

1 so 11.84423

$\begin{array}{lll}2 & \text { so } & 12,00423 \\ 3 & \text { so } & 42,00423\end{array}$

mode $n$

imp:n 11110

$\mathrm{ml} 92234.50 \mathrm{c}$

$92235.50 \mathrm{c}$

$92238.50 \mathrm{C}$

$92236.50 \mathrm{C}$

$8016.50 \mathrm{c}$

$100150 \mathrm{c}$

mt1 lwtr.01t

intr.01t

$\begin{array}{ll}m 2 & 13027.50 \mathrm{c} \\ \mathrm{m} 3 & 1001.50 \mathrm{c}\end{array}$

$8016.50 \mathrm{c}$

mt3 lwtr.01t

$\begin{array}{lllll}\text { kcode } 1000 & 1.0 & 10 & 100 \\ \text { ksrc } 000 & & \end{array}$

print

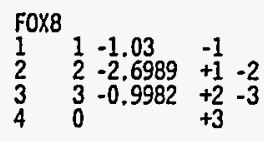

1 so 27.91322

5.70743-6

$5.09183-4$

$.97291-5$

1.46916-6

.

6.44146-2

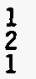

$\frac{1}{2}$ .

政

SPHERE OF URANYL FLUORIDE

TERE OF ALUMINUM

SZERO IMPORTANCE OUTSIDE WDRLD

SSPHERE OF URANYL FLUORIDE

SSPHERE OF ALUMINUH

SWATER REFLECTOR

SZERO IMPORTANCE OUTSIDE WORLD

SSPHERE OF URANYL FLUORIDE

SSPHERE OF ALUMINUM

SZERO IMPORTANCE OUTSIOE WORLD 
$2 \quad 50 \quad 28.11322$

mode $n$

imp:n 1111

ml $92234.50 \mathrm{c}$

92235.500

$92238.50 \mathrm{c}$

$92236.50 \mathrm{c}$

$8016.50 \mathrm{c}$

$1001.50 \mathrm{c}$

$5.89850-7$

$5.26229-5$

$3.07244-6$

3.35955-2

$1.12874-4$

mtl liwtr.01t

6.69653-2

$\mathrm{m} 2 \quad 13027.50 \mathrm{c}$

$3 \quad 1001.50 \mathrm{c}$

mt3 lwtr.01t

$\frac{1}{2}$

kcode 10001.010100

ksrc 000

print

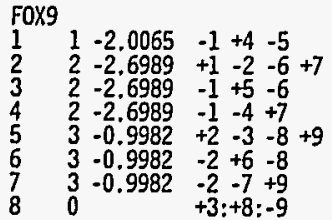

SCYLINOER OF URANYL FLUORIDE SCYLINDER OF ALUMINUM

SALUMINUM (TOP)

SALUMINUM (BOTTOM)

SWATER REFLECTOR

SWATER REFLECTOR (TOP)

SWATER REFLECTOR (BOTTOM)

SZERO IMPORTANCE OUTSIOE KORLD

$\begin{array}{lll}1 & \mathrm{cz} & 7.62 \\ 2 & \mathrm{cz} & 7.7724 \\ 3 & \mathrm{cz} & 37.7724 \\ 4 & \mathrm{pz} & 0.0 \\ 5 & \mathrm{pz} & +89.3 \\ 6 & \mathrm{pz} & +89.4524 \\ 7 & \mathrm{pz} & -0.1524 \\ 8 & \mathrm{pz} & +119.4524 \\ 9 & \mathrm{pz} & -30.1524\end{array}$

mode $n$

imp: $n 16 r 0$

ml 92234.50c

$92235.50 \mathrm{c}$

$92238.50 \mathrm{C}$

$92236.50 \mathrm{c}$

$8016.50 \mathrm{c}$

$1001.50 \mathrm{c}$

mt1 lwtr.olt

5.69969-2

$\begin{array}{lrr}m 2 & 13027.50 \mathrm{c} & 1 \\ \mathrm{~m} 3 & 1001.50 \mathrm{c} & 2 \\ & 8016.50 \mathrm{c} & 1\end{array}$

mt3 lwtr.01t

kcode 10001.010100

ksre 005

print

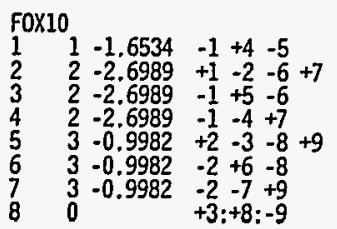

SCYLINDER OF URANYL FLUORIDE

SCYL.INDER OF ALUMINUM

SALUMINUM (TOP)

SALUMINUM (BOTTOM)

SWATER REFLECTOR

SWATER REFLECTOR (TOP)

SWATER REFLECTOR (BOTTOM)

SZERO IMPORTANCE OUTSIDE WORLD

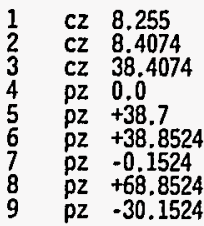

mode $n$

imp: 1 1 $6 r$ r 0

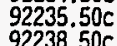

$92238.50 \mathrm{c}$

$1.54393-5$ 


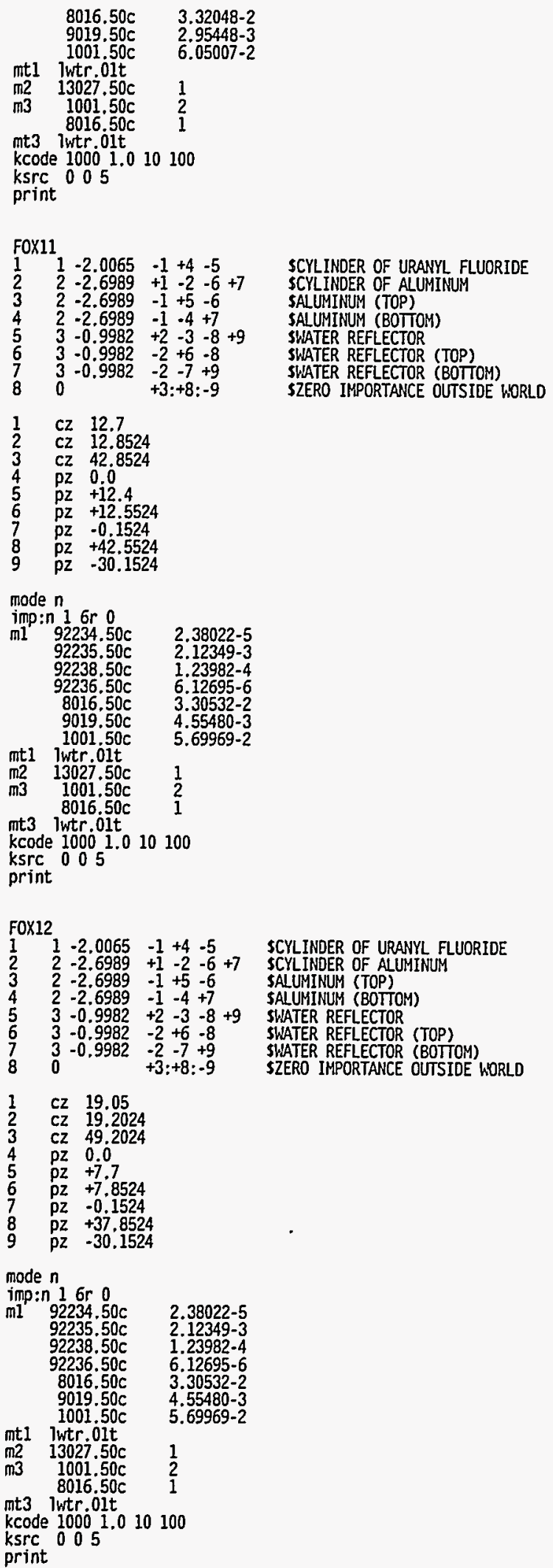




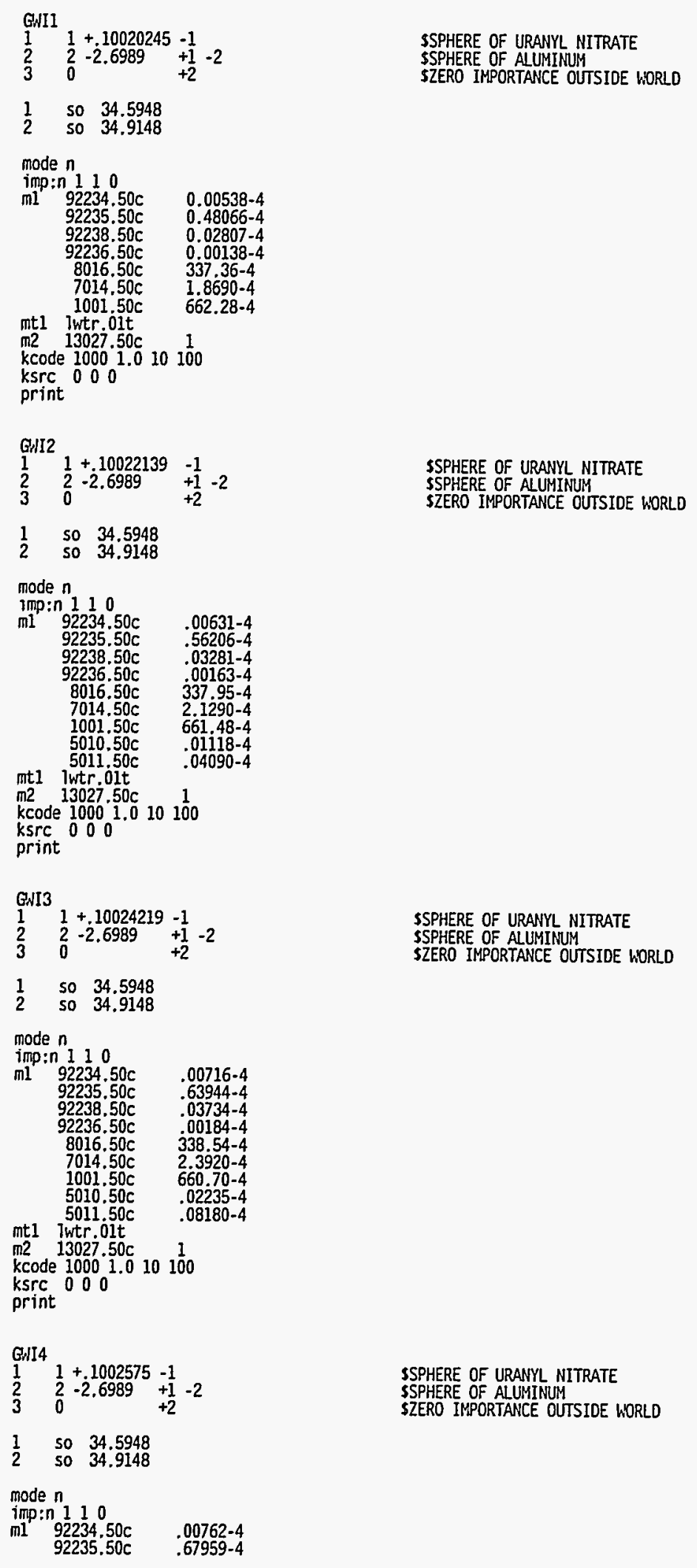

\begin{tabular}{lc} 
Final Report \\
January 1996 & $\begin{array}{c}\text { Validation of } M C N P 4 A \text { on the Battelle } \\
\text { PSRM IBM RS/6000 Workstation }\end{array}$ \\
\hline
\end{tabular}


POEF-SH-33

$.03967-4$

(

$8016.50 \mathrm{c}$

$7014.50 \mathrm{c}$

1001.50

$5010.50 \mathrm{C}$

mtl Iwtr.jit

$.00197-4$

$338.89-4$

$2.5480-4$

$60.28-4$

$.02751-4$
$.10067-4$

$\mathrm{m} 21$ iwtr. $13027.50 \mathrm{c}$

kcode 10001.010100

ksrc 000

print 


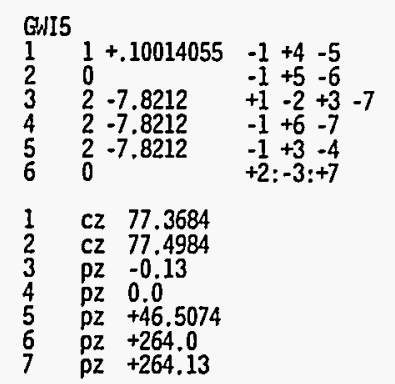

SCYLINDER OF URANYL NITRATE

SCYLINDER VOID

SCYRBOER OF CARBON STEEL

SCARBON STEEL (TOP)

SCARBDN STEEL (BOTTOM)
SZERO IMPORTANCE OUTSIDE WORLD

mode $n$

imp:n $14 r 0$

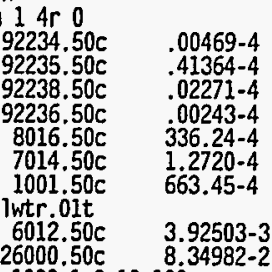

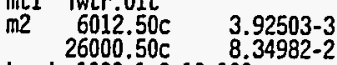

kcode 1000 1.0 10100

ksrc 005

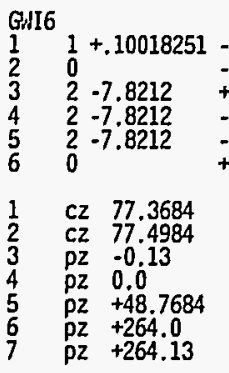

$-1+4-5$
$-1+5-6$ SCYLINDER OF URANYL NITRATE

SCYLINDER OF CARBON STEEL

$1-2+3-7$

$-1+6-7$ SCARBON STEEL (TOP)

$-1+3-4 \quad$ SCARBON STEEL (BOTTOM)

SZERO IMPORTANCE OUTSIDE WORLD

mode $n$

imp:n 1 4r 0

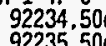

$92235.50 \mathrm{c}$

$92238.50 \mathrm{C}$

$92236.50 \mathrm{c}$

$7014.50 \mathrm{c}$

$100150 \mathrm{c}$

$+2:-3:+7$

mtl lwtr.olt

m2 $\begin{array}{rr}6012.50 \mathrm{c} & 3.92503-3 \\ 26000.50 \mathrm{c} & 8.34982-2\end{array}$

kcode 10001.010100

ksrc 005

print

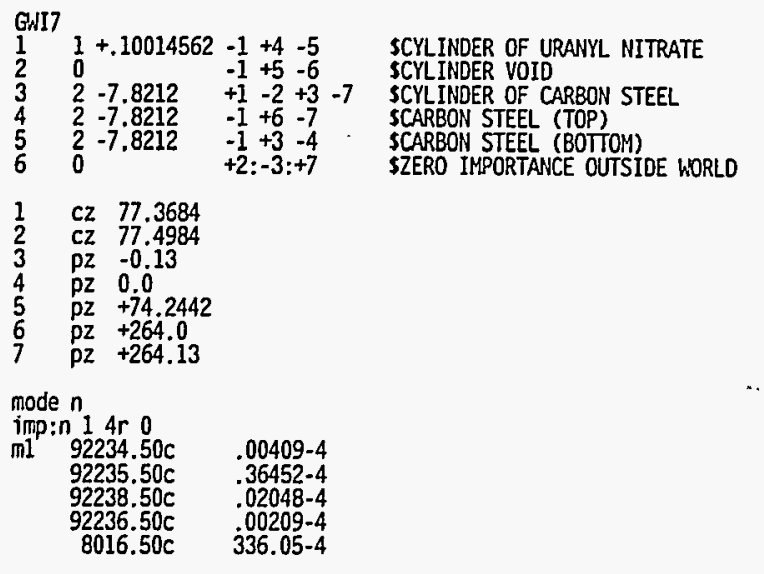




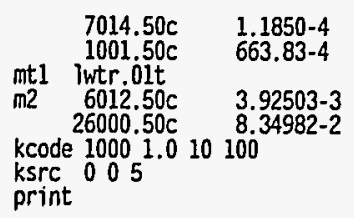

$-1+4-5 \quad$ SCYLINDER OF URANYL NITRATE

SCYLINDER VOIO

SCYLINOER OF CARBON STEEL

SCARBON STEEL (TOP)

SZERO IMPORTANCE OUTSIDE WORLD

mode $n$

$$
\begin{aligned}
& \text { imp: } 1 \text { 1 } 4 r \quad 0 \\
& 92235.50 \mathrm{C} \\
& 92238.50 \mathrm{C} \\
& 92236.50 \mathrm{c} \\
& 8016.50 \mathrm{c} \\
& 7014.50 \mathrm{C}
\end{aligned}
$$

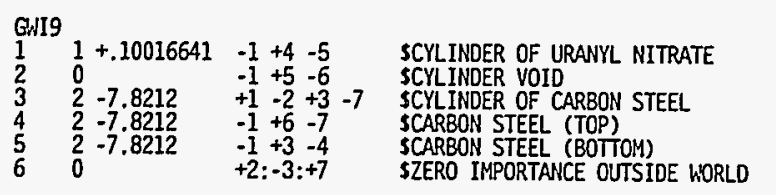

$$
\begin{array}{lll}
1 & \mathrm{cz} & 77.3684 \\
2 & \mathrm{cz} & 77.4984 \\
3 & \mathrm{pz} & -0.13 \\
4 & \mathrm{pz} & 0.0 \\
5 & \mathrm{pz} & +204.9272 \\
6 & \mathrm{pz} & +264.0 \\
7 & \mathrm{pz} & +264.13
\end{array}
$$

\section{mode $n$}

imp:n 1 4r 0

$92234.50 \mathrm{c}$

$92235.50 \mathrm{c}$

$92236.50 \mathrm{c}$

$8016.50 \mathrm{c}$

$7014.50 \mathrm{c}$

1 lwtr 01 6 63.91-4

$\begin{array}{ll}\text { m2 } & \text { W012.50 } \\ 26000.50 \mathrm{c} & 3.92503-3\end{array}$

kcode 10001.010100

ksrc 005

print

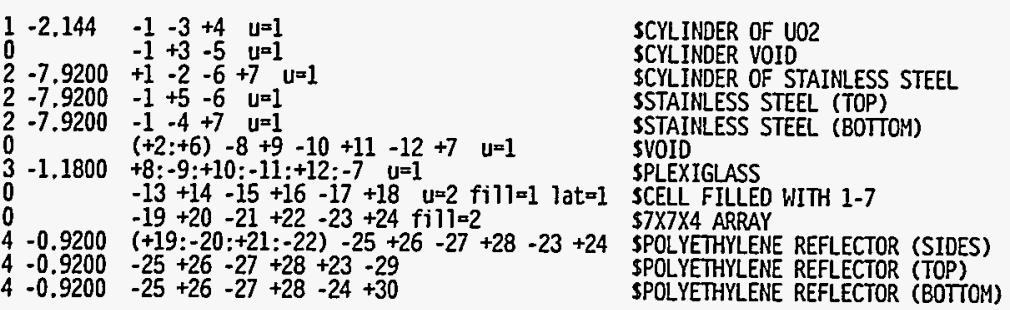

Final Report

January 1996 


$\begin{array}{ll}13 & 0 \\ 1 & c z 3.735 \\ 2 & c z 3.79 \\ 3 & p z+4.535 \\ 4 & p z 0.055 \\ 5 & p z+11.645 \\ 6 & p z+11.70 \\ 7 & p z-0.0001 \\ 8 & p x+4.025 \\ 9 & p x-4.025 \\ 10 & p y+4.025 \\ 11 & p y-4.025 \\ 12 & p z+11.71 \\ 13 & p x+5.195 \\ 14 & p x-5.195 \\ 15 & p y+5.195 \\ 16 & p y-5.195 \\ 17 & p z+14.05 \\ 18 & p z-0.0 \\ 19 & p x+35.194999 \\ 20 & p x-35.194999 \\ 21 & p y+35.194999 \\ 22 & p y-35.194999 \\ 23 & p z+53.859999 \\ 24 & p z+0.0001 \\ 25 & p x+50.195 \\ 26 & p x-50.195 \\ 27 & p y+50.195 \\ 28 & p y-50.195 \\ 29 & p z+68.86 \\ 30 & p z-15.0 \\ & \end{array}$

SZERO IMPORTANCE OUTSIDE MORLD

mode $n$

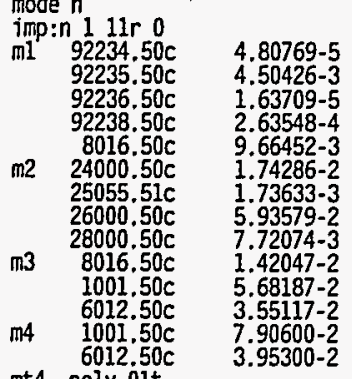

mt4 poly.01t

kcode 10001.010100

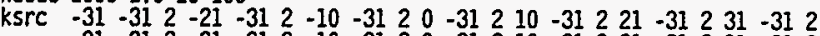

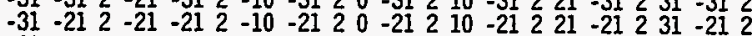

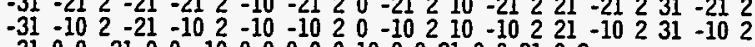

$\begin{array}{llllllllllllllllllll}-31 & 0 & 0 & -21 & 0 & 0 & -10 & 0 & 0 & 0 & 0 & 0 & 10 & 0 & 0 & 21 & 0 & 0 & 31 & 0\end{array}$

$\begin{array}{llllllllllllllllllllll}-31 & 10 & 0 & -21 & 10 & 0 & -10 & 10 & 0 & 0 & 10 & 0 & 10 & 10 & 0 & 21 & 10 & 0 & 31 & 10 & 2\end{array}$

$\begin{array}{lllllllllllllllllllll}-31 & 21 & 0 & -21 & 21 & 0 & -10 & 21 & 0 & 0 & 21 & 0 & 10 & 21 & 0 & 21 & 21 & 0 & 31 & 21 & 2 \\ -31 & 31 & 0 & -21 & 31 & 0 & -10 & 31 & 0 & 0 & 31 & 0 & 10 & 31 & 0 & 21 & 31 & 0 & 31 & 31 & 2\end{array}$

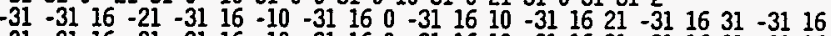

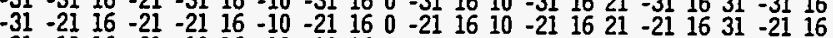

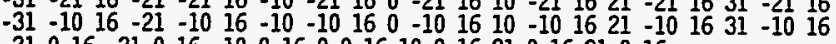

$\begin{array}{lllllllllllllllllllllll}-31 & 0 & 16 & -21 & 0 & 16 & -10 & 0 & 16 & 0 & 0 & 16 & 10 & 0 & 16 & 21 & 0 & 16 & 31 & 0 & 16\end{array}$

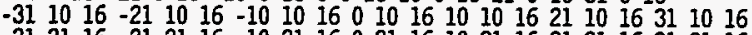

$\begin{array}{lllllllllllllllllllll}-31 & 21 & 16 & -21 & 21 & 16 & -10 & 21 & 16 & 0 & 21 & 16 & 10 & 21 & 16 & 21 & 21 & 16 & 31 & 21 & 16\end{array}$

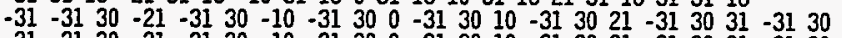

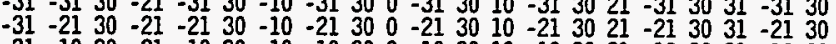

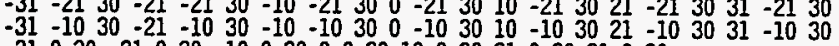

$\begin{array}{lllllllllllllllllllll}-31 & 0 & 30 & -21 & 0 & 30 & -10 & 0 & 30 & 0 & 0 & 30 & -10 & 0 & 30 & 21 & 0 & 30 & 31 & 0 & 30\end{array}$

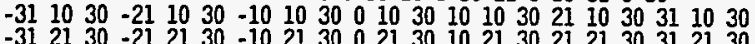

$\begin{array}{lllllllllllllllllllllllll}-31 & 21 & 30 & -21 & 21 & 30 & -10 & 21 & 30 & 0 & 21 & 30 & 10 & 21 & 30 & 21 & 21 & 30 & 31 & 21 & 30 \\ -31 & 31 & 30 & -21 & 31 & 30 & -10 & 31 & 30 & 0 & 31 & 30 & 10 & 31 & 30 & 21 & 31 & 30 & 31 & 31 & 30\end{array}$

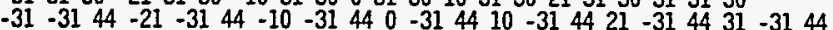

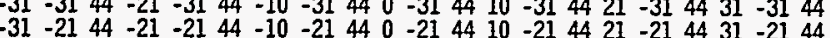

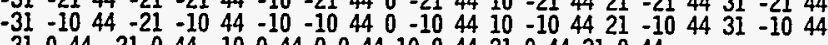

-31 $044-21044-100440044100442104431044$

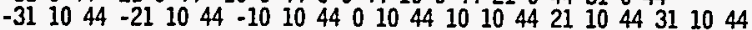

$\begin{array}{llllllllllllllllllllllll}-31 & 21 & 44 & -21 & 21 & 44 & -10 & 21 & 44 & 0 & 21 & 44 & 10 & 21 & 44 & 21 & 21 & 44 & 31 & 21 & 44 \\ -31 & 31 & 44 & -21 & 31 & 44 & -10 & 31 & 44 & 0 & 31 & 44 & 10 & 31 & 44 & 21 & 31 & 44 & 31 & 31 & 44\end{array}$ print

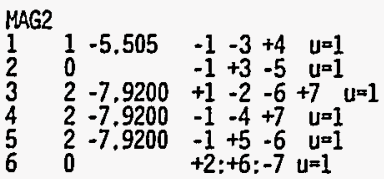

SCYLINDER OF VO2
\$CYLINDER VOID
SSTAINLESS STEEL CONTAINER
SSTAIILESS STEEL (TOP)
SSTAINLESS STEEL (BOTOM)
SVOIDLES

SVOID 


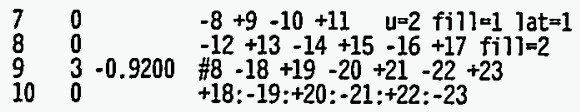

$\begin{array}{ll}1 & c z 7.65 \\ 2 & c z 7.68 \\ 3 & p z+8.88 \\ 4 & p z-10.88 \\ 5 & p z+10.88 \\ 6 & p z+10.91 \\ 7 & p z-10.91 \\ 8 & p x+7.85 \\ 9 & p x-7.85 \\ 10 & p y+7.85 \\ 11 & p y-7.85 \\ 12 & p x+54.949999 \\ 13 & p x-7.849999 \\ 14 & p y+54.949999 \\ 15 & p y-7.849999 \\ 16 & p z+11.15 \\ 17 & p z-11.15 \\ 18 & p x+69.95 \\ 19 & p x-22.85 \\ 20 & p y+69.95 \\ 21 & p y-22.85 \\ 22 & p z+26.15 \\ 23 & p z-26.15\end{array}$

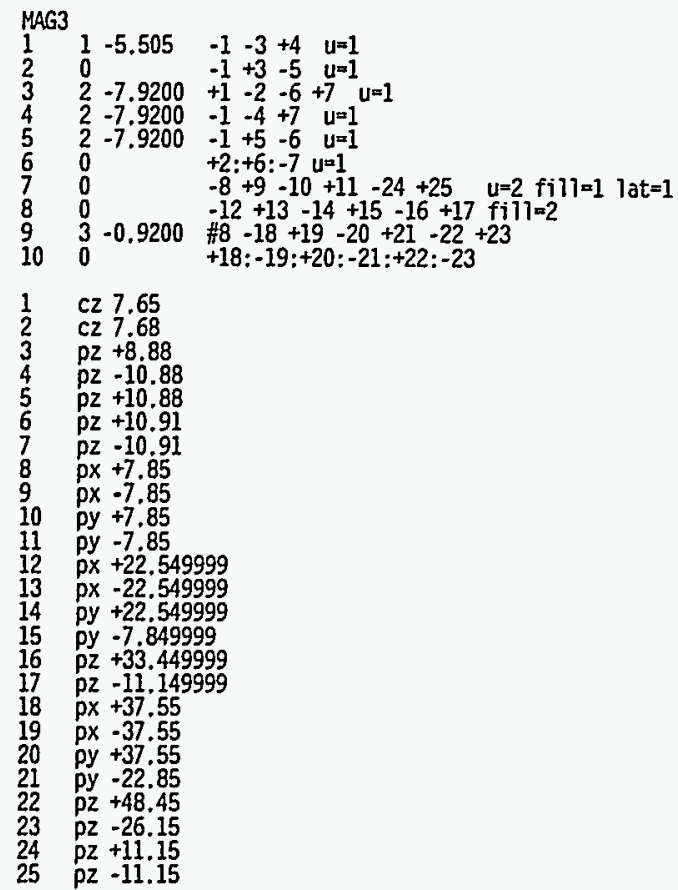

SCELL FILLED WITH 1-6

SPOLYETHYLENE REFLECTOR

SZERO IMPORTANCE OUTSIOE WORLD

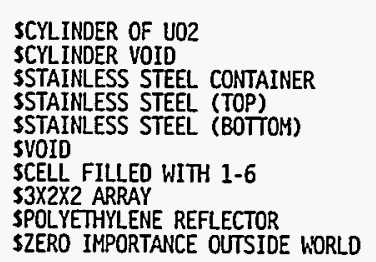

SCYLINDER VOID

SSTAINLESS STEEL CONTAINER

STAINLESS STEEL (TOP)

SSTAINLESS STEEL (BOTTOM)

SPOLYETHYLENE REFLECTOR

SZERO IMPORTANCE OUTSIDE WORLD 
mode $n$

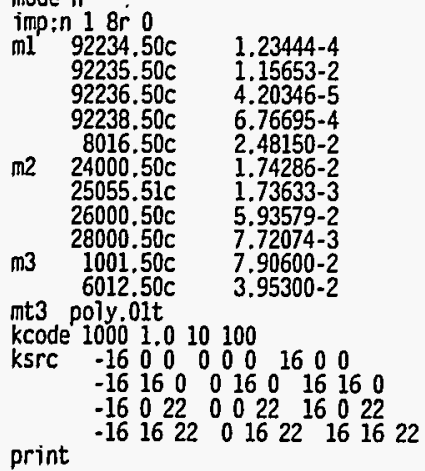

SCYLINDER OF U02

SCYLINDER VOID

SSTAINLESS STEEL CONTAINER

SSTAINLESS STEEL (TOP)

SSTAINLESS STEEL (BOTTOM)

SVOID

\$CELL FILLED WITH 1.6

\$3X3X2 ARRAY

SPOLYETHYLENE REFLECTOR

\$ZERO IMPORTANCE OUTSIDE WORLD

\section{$\begin{array}{lll}1 & c z & 7.65 \\ 2 & c z & 7.68\end{array}$ \\ pz -10.88 \\ pz +10.88 \\ Pz +10.91 \\ pz -10.91 \\ px +9.08 \\ py +9.08 \\ py -9.08 \\ $\mathrm{px}+27.239999$ \\ py +27.239999 \\ pz +33.449999 \\ pz -11.149999 \\ $\mathrm{px}+42.24$ \\ px -42.24 \\ py +42.24 \\ $\mathrm{pZ}+48.45$ \\ pz -26.15 \\ $p Z+11.15$}

mode

imp: $n 18 r \quad 0$

$\mathrm{ml} 92234.50 \mathrm{c}$

(2)

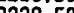

2238.50

$+9-10+11-24+25 \quad u=2$ fil

$48-18+19+15-16+17$ fj 1

$\# 8-18+19-20+21-22+23$
$+18:-19:+20:-21:+22:-23$

m2

240005

$24000.50 \mathrm{c}$

$600050 \mathrm{c}$

$0000.50 \mathrm{C}$

100150

$6012.50 \mathrm{c}$

mt3 poly.01t

kcode $1000 \quad 1.010100$

$\begin{array}{lllllllllll}\text { ksrc } & -18 & -18 & 0 & 0 & -18 & 0 & 18 & -18 & 0\end{array}$

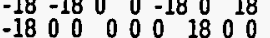

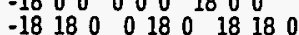

$\begin{array}{lllllllll}-18 & 18 & 0 & 0 & 18 & 0 & 18 & 18 & 0 \\ -18 & -18 & 22 & 0 & -18 & 22 & 18 & -18 & 22\end{array}$

print

1.23444-4

$1.15653-2$

$4.20346-5$

.48150-2

$1.74286-2$

$5.93579-2$

7.72074-3

$7.90600-2$

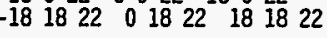

$\begin{array}{lllllll}\text { MAG55 } & & & & \\ 1 & 1 & -5.505 & -1 & -3 & +4 & u=1 \\ 2 & 0 & & -1 & +3 & -5 & u=1 \\ 3 & 2 & -7.9200 & +1 & -2 & -6 & +7 \\ 4 & 2 & -7.9200 & -1 & +5 & -6 & u=1\end{array}$

\section{Final Report}

January 1996 
mode $n$

imp:n $18 r 0$

ml $92234.50 \mathrm{c}$

$92235.50 \mathrm{c}$ $92236.50 \mathrm{c}$ $92238.50 \mathrm{c}$

m2 $8016.50 \mathrm{c}$ $25055.51 \mathrm{c}$ $26000.50 \mathrm{c}$ $28000.50 \mathrm{c}$

$\mathrm{m} 3$ $1001.50 \mathrm{c}$

mt3 poly.01t

1.23444-4

$1.15653-2$

$4.20346-5$

$6.76695-4$

$2.48150-2$

$1.74286-2$

$1.73633-3$

$5.93579-2$

$7.72074-3$

$3.90600-2$

kcode 10001.010100

ksrc -16000000160

$\begin{array}{llllllll}-16 & 160 & 0 & 16 & 0 & 16 & 160\end{array}$

$\begin{array}{lllllllll}-16 & 0 & 22 & 0 & 0 & 22 & 16 & 0 & 22 \\ -16 & 16 & 22 & 0 & 16 & 22 & 16 & 16 & 22\end{array}$

print

MAG4

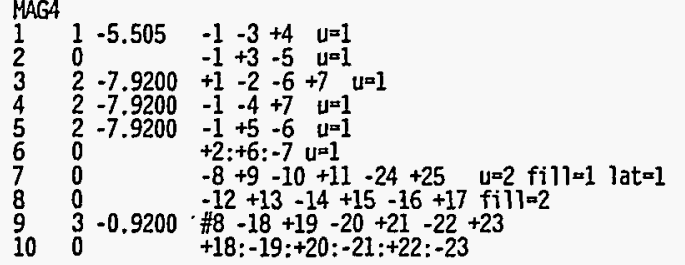

SCYLINDER OF U02

SYYINDER VOID

STAINLESS STEEL CONTAINER

SSTATNL ESS STEEL (TOP)

STAINLESS STEEL (BOTTOM)

SVOID

SCELL FILLED WITH 1-6

\$3X3X2 ARRAY

SPOLYETHYLENE REFLECTOR

SZERO IMPORTANCE OUTSIDE WORLD

\footnotetext{
$\frac{1}{2} \quad$ cz 7.65

C2 7.68

pz -10.88

$\mathrm{pz}-10.88$

$p Z+10.88$

$p z+10.91$

$\mathrm{px}+9.08$

$p x-9.08$

py +9.08

11 py -9.08

$12 \mathrm{px}+27.23999$

$13 \mathrm{px}-27.239999$

14 py +27.239999

py -27.2399

17 pz -11.149999

$18 \mathrm{px}+42.28$

19 pX $-42,24$

$19 \quad \mathrm{px}-42.24$

py +42.24

Py -42.24

$23 \quad \mathrm{DZ}-26.15$

pz +11.15

$25 \mathrm{pz}-11.15$
}

mode $n$

imp: $n 18 r 0$

m1

$92234.50 \mathrm{c}$
$92235.50 \mathrm{c}$

$92236.50 \mathrm{c}$

$8238.50 \mathrm{c}$

m2 $24000.50 \mathrm{c}$

25055.51

$26000.50 \mathrm{C}$

$\mathrm{m3} \quad 1001.50 \mathrm{c}$

$6012.50 \mathrm{C}$

mt3 poly.01t

1.23444-4

1.15653-2

$6.76695-4$

$2.48150-2$

$2.48150-2$

$1.74286-2$

$1.73633-3$

$5.93579-2$

.

$3.95300-2$

kcode 1000 I. 010100

$\begin{array}{llllllllll}\text { ksrc } & -18 & -18 & 0 & 0 & -18 & 0 & 18 & -18 & 0\end{array}$

$\begin{array}{lllllllll}-18 & 0 & 0 & 0 & 0 & 0 & 18 & 0 & 0\end{array}$

$\begin{array}{lllllllll}-18 & 18 & 0 & 0 & 18 & 0 & 18 & 18 & 0\end{array}$

$\begin{array}{lllllllll}-18 & -18 & 22 & 0 & -18 & 22 & 18 & -18 & 22\end{array}$

$-18022002218022$

print

$.181822 \quad 01822 \quad 181822$

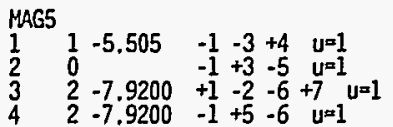

SCYLINDER OF UO2

SCYLINDER VOID

SCYLINDER OF STAIIILESS STEEL

SSTAINLESS STEEL (TOP)

Final Report

January 1996

Validation of MCNP4A on the Battelle

Page 31

PSRM IBM RS/6000 Workstation 

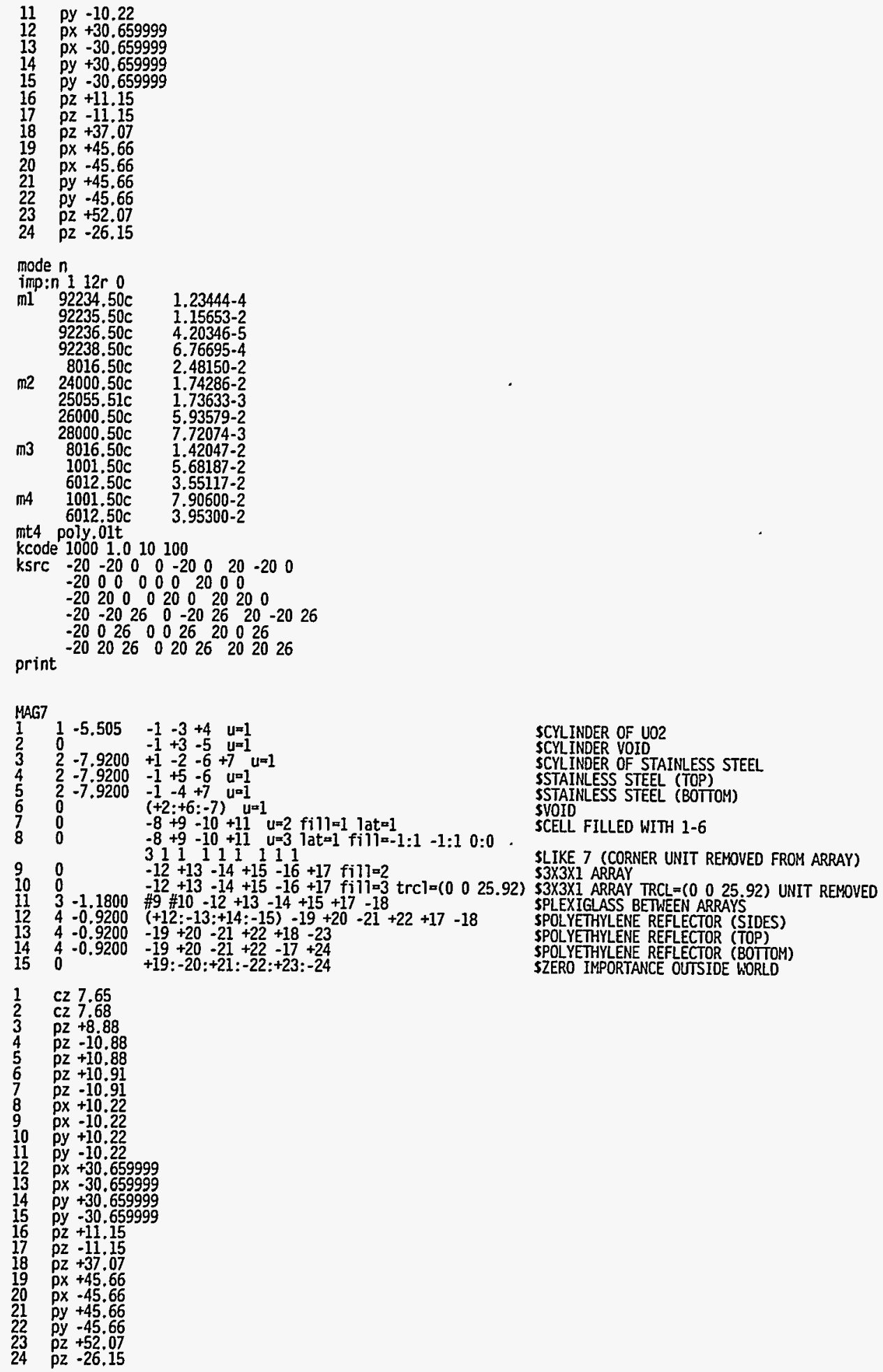

mode $n$

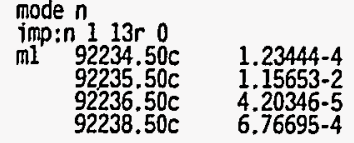




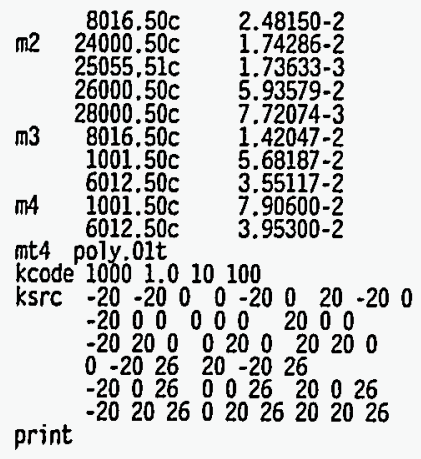

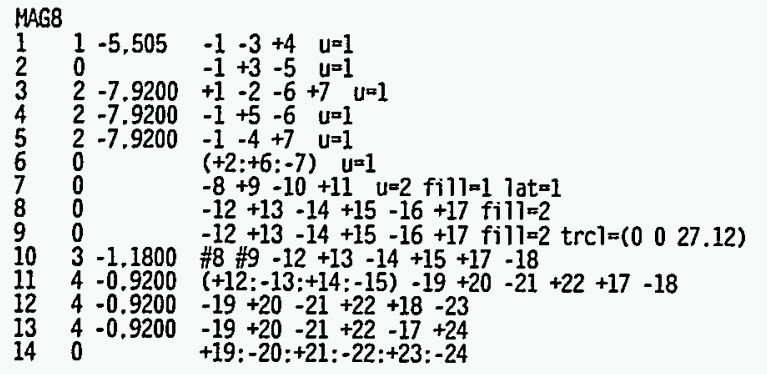

$\begin{array}{ll}1 & c z 7.65 \\ 2 & c z 7.68 \\ 3 & p z+8.88 \\ 4 & p z-10.88 \\ 5 & p z+10.88 \\ 6 & p z+10.91 \\ 7 & p z-10.91 \\ 8 & p x+10.22 \\ 9 & p x-10.22 \\ 10 & p y+10.22 \\ 11 & p y-10.22 \\ 12 & p x+30.659999 \\ 13 & p x-30.659999 \\ 14 & p y+30.659999 \\ 15 & p y-30.659999 \\ 16 & p z+11.15 \\ 17 & p z-11.15 \\ 18 & p z+38.27 \\ 19 & p x+45.66 \\ 20 & p x-45.66 \\ 21 & p y+45.66 \\ 22 & p y-45.66 \\ 23 & p z+53.27 \\ 24 & p z-26.15 \\ & \end{array}$

SCYLINDER OF U02 SCYLINDER VOID \$CYLINDER OF STAINLESS STEEL SSTAINLESS STEEL (TOP) SSTAINLESS STEEL (BOTTOM) SVOID SCELL FILLEO WITH 1-6 \$3X3X1 ARRAY \$3X3X1 ARRAY
S3X3X1 ARRAY TRCL $=\left(\begin{array}{lll}0 & 0 & 27.12\end{array}\right)$ SPLEXIGLASS BETWEEN ARRAYS \$POLYETHYLENE REFLECTOR (SIDES) SPOLYETHYLENE REFLECTOR (TOP) SPOLYETHYLENE REFLECTOR (BOTTOM)

print

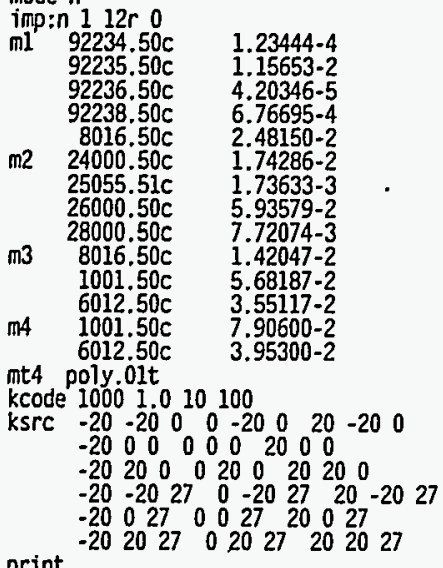

MAG9 


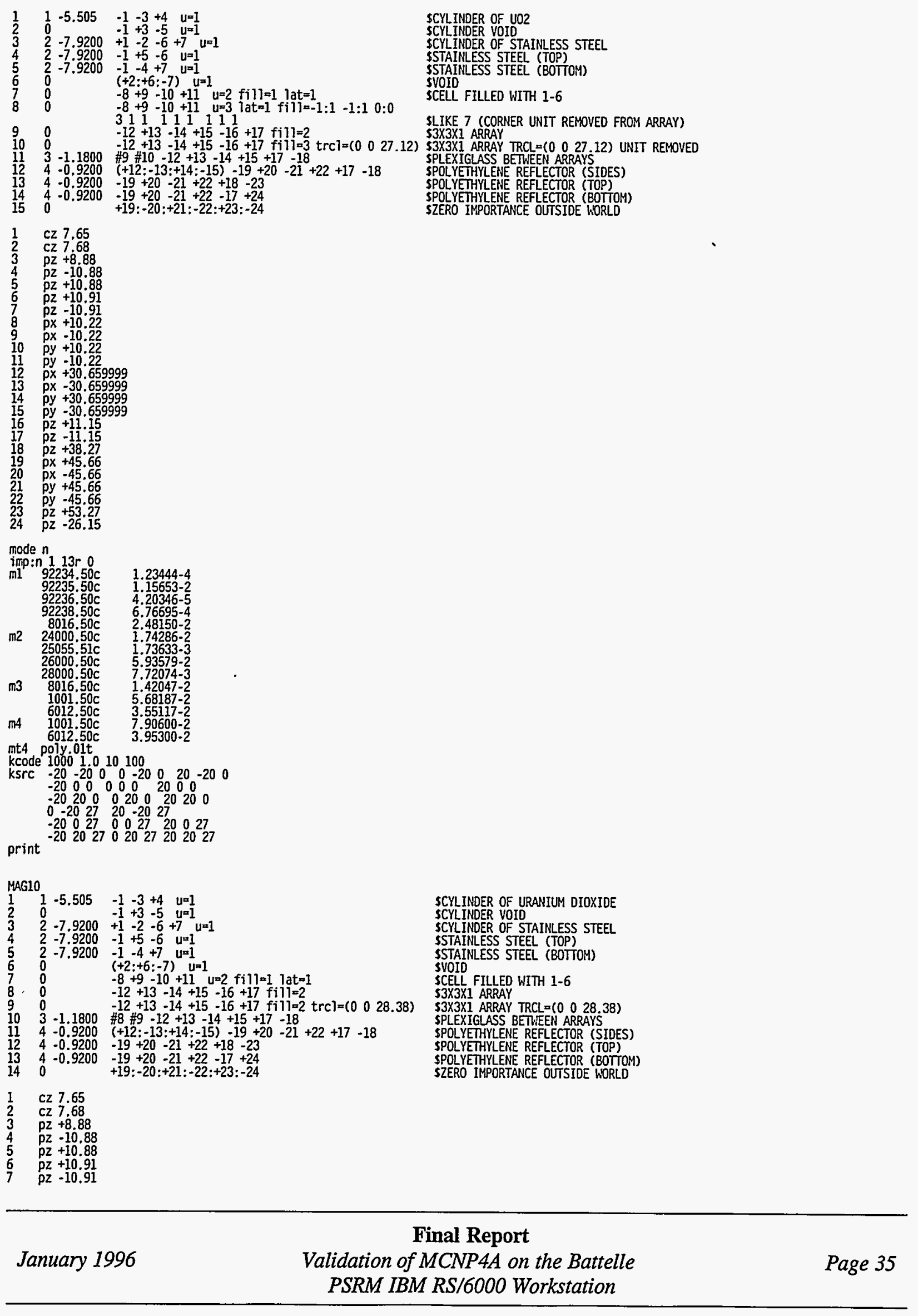




$$
\begin{array}{ll}
8 & p x+10.22 \\
9 & p x-10.22 \\
10 & p y+10.22 \\
11 & p y-10.22 \\
12 & p x+30.659999 \\
13 & p x-30.659999 \\
14 & p y+30.659999 \\
15 & p y-30.659999 \\
16 & p z+11.15 \\
17 & p z-11.15 \\
18 & p z+39.53 \\
19 & p x+45.66 \\
20 & p x-45.66 \\
21 & p y+45.66 \\
22 & p y-45.66 \\
23 & p z+54.53 \\
24 & p z-26.15
\end{array}
$$

mode $n$

$$
\begin{aligned}
& \text { imp: }{ }^{2} 112 r \quad 0 \\
& 92235.50
\end{aligned}
$$

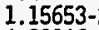

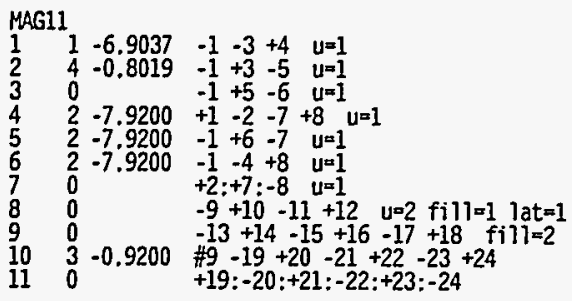

$1 \quad$ CZ 6.76

CZ 6.79

pz +5.27

pz -12.60

$\mathrm{pz}+8.41$

$p z+12.58$

PZ +12.63

PZ -12 .

$\mathrm{PX}+6.985$

$\mathrm{px}+6.985$

px -6.985

py +6.985

py -6.985

$\mathrm{px}+20.954999$

pX -6.984999

py +20.954999

py -6.984999

PZ +12.78

$\mathrm{pz}-12.78$

$\mathrm{px}+35,955$

$\mathrm{px}-21.985$

py +35.955

py -21.985

$p z-27.78$

mode $n$

imp: n 1 9r 0

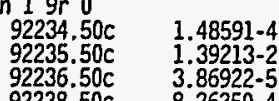

SCYLINDER OF URANIUM DIOXIDE WITH ALCOHOL

SALCOHOL LAYER ABOVE OXIDE

SYOID

SSTAINLESS STEEL CONTAINER

SSTAMLESS STEEL (TOP)

SWTAIDESS STEEL (BOTTOM)

SVOID

SCELL FILLED WITH 1-7

$\$ 2 X 2 X 1$ ARRAY

SPOLYETHYLENE REFLECTOR

SZERO IMPORTANCE OUTSIDE WORLD 


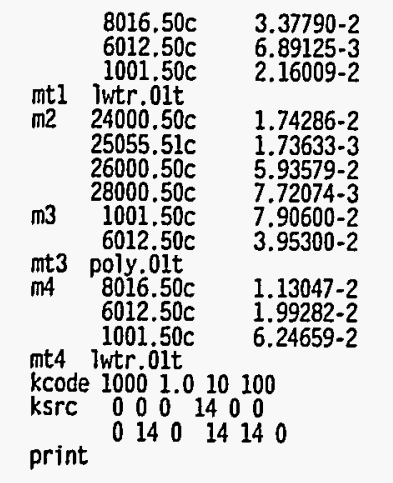

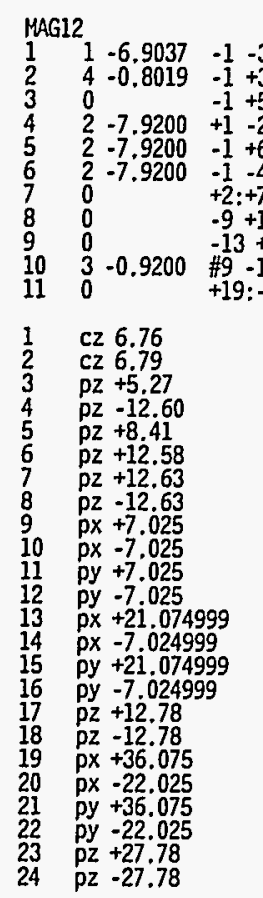

mode $n$

mode $n$.

imp: 1 9r 0

ml 92234.50

$92235.50 \mathrm{c}$

92238.50

$8016.50 \mathrm{C}$

$6012,50 \mathrm{c}$
$1001.50 \mathrm{C}$

mt1 Iwtr.01t

$1.48591-4$

$\frac{1}{3.39213-2}$

8.26350 .4

$3.26790-2$

$6.89125-3$

$1.74286-2$

$\begin{array}{ll}24000.50 \mathrm{C} & 1.74286-2 \\ 25055.51 \mathrm{C} & 1.73633-3\end{array}$

$26000.50 \mathrm{C}$

$28000.50 \mathrm{C}$

$\mathrm{m3}$

$1001.50 \mathrm{c}$

t3 poly.01t

$8016.50 \mathrm{c}$

8016.50

$1001.50 \mathrm{C}$

$5.93579-2$

$7.72074-3$

$3.95300-2$

$13047-2$

$1.13047-2$

$6.24659-2$

mt4 iwtr.01t

kcode 10001.010100

ksrc $\begin{array}{lllllllll}0 & 0 & 0 & 14 & 0 & 0 \\ 0 & 14 & 0 & 14 & 14\end{array}$

print

$\begin{array}{lllllll}\text { MAG13 } & & & & & \\ 1 & 1 & -6.9037 & -1 & -3 & +4 & u=1 \\ 2 & 4 & -0.8019 & -1 & +3 & -5 & u=1\end{array}$
SCYLINDER OF URANIUM DIOXIDE WITH ALCOHOL SALCOHOL LAYER ABOVE OXIDE

SSTAINLESS STEEL CONTAINER

SSTAINLESS STEEL (TOP)

SSTAINLESS STEEL (BOTTOM)

SVOID

SCELL FILLED WITH 1-7

$\$ 2 \times 2 \times 1$ ARRAY

SPOLYETHYLENE REFLECTOR

SZERO IMPORTANCE OUTSIDE WORLD 


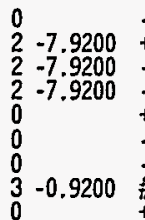

$-1+5-6 \quad u=1$

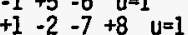

$-1+6-7 \quad u=1$

$\begin{array}{lll}-1 & -4 & -7 \\ + & \mathrm{u}=1\end{array}$

$-9+10-11+12 \quad u=2$ fill $=1 \quad$ lat $=1$

$-13+14-15+16-17+18$ fill $=2$

\#9 $-19+20-21+22-23+24$

$\begin{array}{ll}1 & c z 6.76 \\ 2 & c z 6.79 \\ 3 & p z+5.27 \\ 4 & p z-12.60 \\ 5 & p z+8.41 \\ 6 & p z+12.58 \\ 7 & p z+12.63 \\ 8 & p z-12.63 \\ 9 & p x+7.064 \\ 10 & p x-7.064 \\ 11 & p y+7.064 \\ 12 & p y-7.064 \\ 13 & p x+21.191999 \\ 14 & p x-7.063999 \\ 15 & p y+21.191999 \\ 16 & p y-7.063999 \\ 17 & p z+12.78 \\ 18 & p z-12.78 \\ 19 & p x+36.192 \\ 20 & p x-22.064 \\ 21 & p y+36.192 \\ 22 & p y-22.064 \\ 23 & p z+27.78 \\ 24 & p z-27.78\end{array}$

mode $n$

imp: n 1 9r 0

$92234.50 \mathrm{c} \quad 1.48591-4$ $92235.50 c$ $92238.50 \mathrm{c}$ $8016.50 \mathrm{c}$ $6012.50 \mathrm{c}$

100150

mtI Iwtr.01t

24000.50

$25055.51 \mathrm{C}$

$28000.50 \mathrm{c}$

$1001.50 \mathrm{c}$

$6012.50 \mathrm{C}$

mt3 poly.01t

$8016.50 \mathrm{c}$

$1001.50 \mathrm{c}$

1.48591-.

3. $86922-5$

$8.26350-4$

$3.37790-2$

$6.89125-3$

$1.74885-2$

$1.74286-2$

1.73633-3

5. $73579-2$

$7.90600-2$

$3.95300-2$

$1.13047-2$

$1.99282-2$

mt4 lwtr.01t

kcode 10001010100

ksrc 00001400

print

$\begin{array}{llllll}0 & 0 & 0 & 14 & 0 & 0\end{array}$

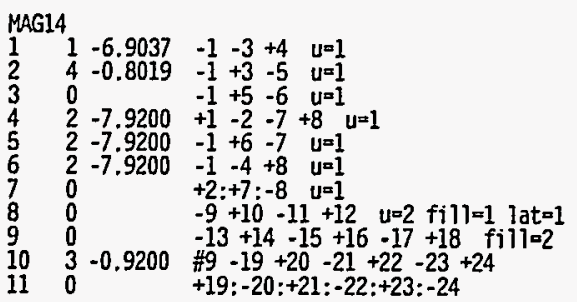

$\begin{array}{ll}1 & \mathrm{cz} 6.76 \\ 2 & \mathrm{cz} 6.79 \\ 3 & \mathrm{pz}+5.27 \\ 4 & \mathrm{pz}-12.60 \\ 5 & \mathrm{pz}+8.41 \\ 6 & \mathrm{pz}+12.58 \\ 7 & \mathrm{pz}+12.63 \\ 8 & \mathrm{pz}-12.63 \\ 9 & \mathrm{px}+7.620 \\ 10 & \mathrm{px}-7.620 \\ 11 & \mathrm{py}+7.620 \\ 12 & \mathrm{py}-7.620 \\ 13 & \mathrm{px}+22.859999 \\ 14 & \mathrm{px}-7.619999\end{array}$
SVOID

SSTAINLESS STEEL CONTAINER

SSTAINLESS STEEL (TOP)

SSTAINLESS STEEL (BOTTOM)

SVOTD

SCELL FILLED WITH 1-7

52X2X1 ARRAY

SPOLYETTYYLENE REFLECTOR

SZERO IMPORTANCE OUTSIDE WORLD
SCYLINDER OF URANIUM DTOXIDE WITH ALCOHOL

SALCOHOL LAYER ABOVE OXIDE

SVOID

SSTAINLESS STEEL CONTAINER

\STAINLESS STEEL (TOP)

SSTAINLESS STEEL (BOTTOM)

SVOID

SCELL FILLED WITH 1.7

\$2X3XI ARRAY

SPOLYETHYLENE REFLECTOR

SZERO IMPORTANCE OUTSIDE WORLD

\section{January 1996}




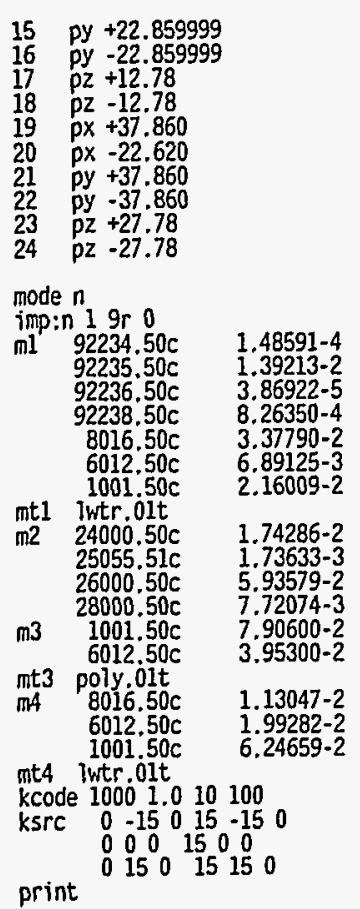

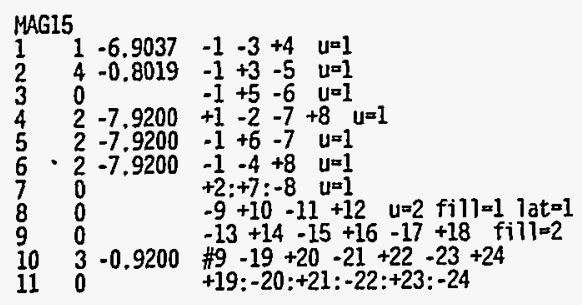

$\begin{array}{ll}1 & c z 6.76 \\ 2 & c z 6.79 \\ 3 & p z+5.27 \\ 4 & p z-12.60 \\ 5 & p z+8.41 \\ 6 & p z+12.58 \\ 7 & p z+12.63 \\ 8 & p z-12.63 \\ 9 & p x+7.938 \\ 10 & p x-7.938 \\ 11 & p y+7.938 \\ 12 & p y-7.938 \\ 13 & p x+23.813999 \\ 14 & p x-7.937999 \\ 15 & p y+23.813999 \\ 16 & p y-23.813999 \\ 17 & p z+12.78 \\ 18 & p z-12.78 \\ 19 & p x+38.814 \\ 20 & p x-22.938 \\ 21 & p y+38.814 \\ 22 & p y-38.814 \\ 23 & p z+27.78 \\ 24 & p z-27.78\end{array}$
SCYLINDER OF URANIUM DIOXIDE WITH ALCOHOL

SALCOHOL LAYER ABOVE OXIDE

SVOID

SSTAINLESS STEEL CONTAINER

SSTAIMLESS STEEL (TOP)

SSTAINLESS STEEL (BOTTOM)

SCELL FILLED WITH 1-7

\$2X3X1 ARRAY

SPOLYTHYLENE REFLECTOR

SZERO IMPORTANCE OUTSIDE WORLD

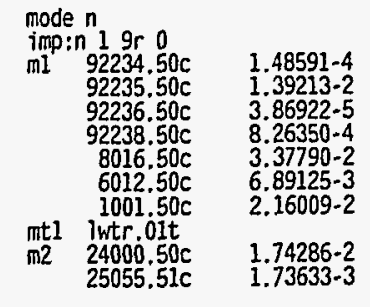

Final Report

January 1996 


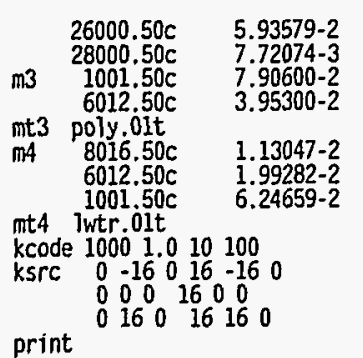

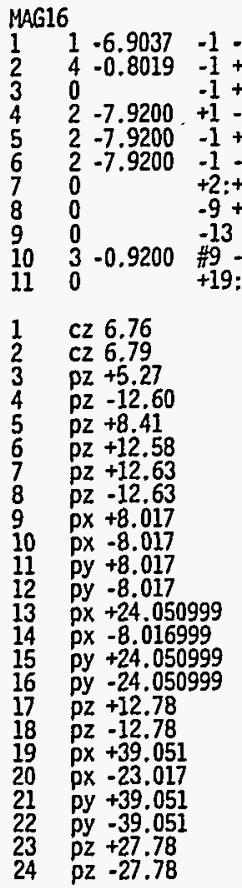

mode $n$

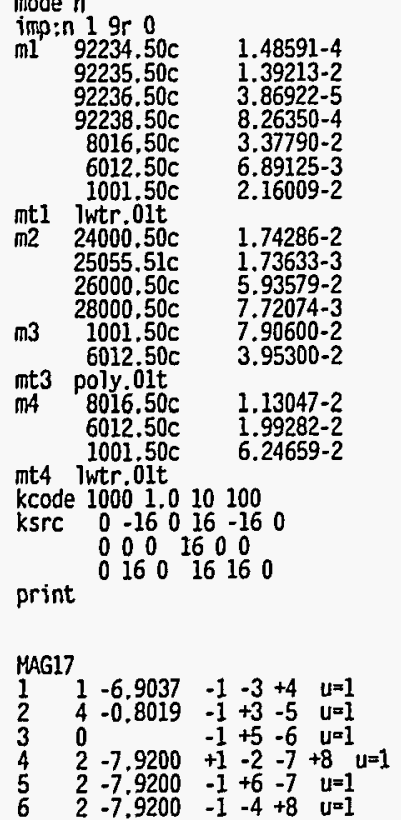

SCYLINDER OF URANIUM DTOXIDE WITH ALCOHOL SALCOHOL LAYER ABOVE OXIDE

SVIOTD

SSTA

STAIMIESS STEEI (TOP)

SSTAIMESS STEEL (TOP)
SSTAIMLESS STEEL (BOTTOM)

SVOID

SCELL FILLED WITH 1-7

S2X3X1 ARRAY

SPOLYETHYLENE REFLECTOR

SZERO IMPORTANCE OUTSIDE WORLD

SCYLINDER OF URANIUM DIOXIOE WITH ALCOHOL

SALCOHOL LAYER ABOVE OXIDE

SVOID

SSTAINLESS STEEL CONTAINER

SSTAIMLESS STEEL (TOP)

SSTAINLESS STEEL (BOTIOM)

\section{Final Report}

January 1996 


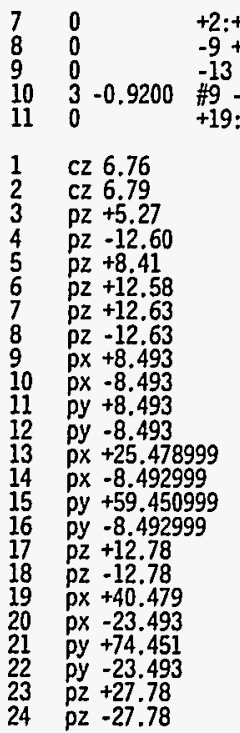

mode $n$

imp: 1 gr 0

ml :n 1 gr 0

$92234.50 \mathrm{c}$

$92235.50 \mathrm{c}$

$92238.50 \mathrm{c}$

$8016.50 \mathrm{c}$

政

$100150 \mathrm{c}$

$+2:+7:-8 \quad u=1$

$-9+10-11+12 \quad u=2 \quad$ fill $=1$ lat=l

$-13+14-15+16-17+18$ fil $11=2$

$\# 9-19+20-21+22-23+24$
$+19:-20:+21:-22:+23:-24$

lwtr.01t

$\mathrm{m}_{\mathrm{m} 2}$

$26000.50 \mathrm{c}$

$\mathrm{m} 3$

5. $93579-2$

$7.90600-2$

int poly.01t

$8016.50 \mathrm{c}$

$1001.50 \mathrm{c}$

mt4 lwtr.01t

kcode 10001.010100

ksrc 00001700

$\begin{array}{lllllllll}0 & 17 & 17170\end{array}$

$\begin{array}{llllll}0 & 34 & 0 & 17 & 17 & 0 \\ 0 & 34 & 0\end{array}$

print
$1.74286-2$

1.48591-4

$86213-2$

$8.26350-4$

(8)

$1.13047-2$

. $.99282-2$
SVOID

SCELL FILLED WITH 1-7

S2X4X1 ARRAY

SPOLYETHYLENE REFLECTOR

SZERO IMPORTANCE OUTSIDE WORLD

MAG18

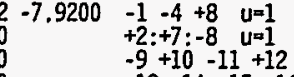

$-9+10 \quad-11+12 \quad u=2$ fillel lat=1
$-13+14-15+16-17+18 \quad$ fill $=2$

$-0.9200$

$-13+19+20-21+22-23+24$
$+19:-20:+21:-22:+23:-24$

$1 \quad c z 6.76$

C2 6.79

pz +5.27

pz $-12,60$

$p Z+8,41$

$\mathrm{pz}+12.58$

$\mathrm{pz}+12.63$

$\mathrm{pz}-12.63$

$p x+9.049$

px -9.049

py +9.049

py -9.049

px -27.146999

py +27.146999

py +27.146999
py -27.146999

SCYLINDER OF URANIUM OIOXIDE WITH ALCOHOL

SALCOHOL LAYER ABOVE OXIDE

SVOID

SSTAINLESS STEEL CONTAINER

SSTAIMLESS STEEL (TOP)

SSTAINLESS STEEL (BOTTOM)

SVOID

SCELL FILLED WITH 1-7

$53 \times 3 \times 1$ ARRAY

SPOLYETHYLENE REFLECTOR

SZERO IMPORTANCE OUTSIDE WORLD 


$$
\begin{array}{ll}
17 & p z+12.78 \\
18 & p z-12.78 \\
19 & p x+42.147 \\
20 & p x-42.147 \\
21 & p y+42.147 \\
22 & p y-42.147 \\
23 & p z+27.78 \\
24 & p z-27.78
\end{array}
$$

mode $n$

$$
\begin{aligned}
& \text { imp: }{ }^{2} 19 r \quad 0 \\
& 92235.50 c \\
& 92238.50 \mathrm{c}
\end{aligned}
$$

kcode 10001010100

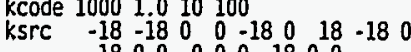
$\begin{array}{llllllllll}-18 & 18 & 0 & 0 & 0 & 18 & 0 & 0 \\ .18 & 18 & 18 & 0\end{array}$

print

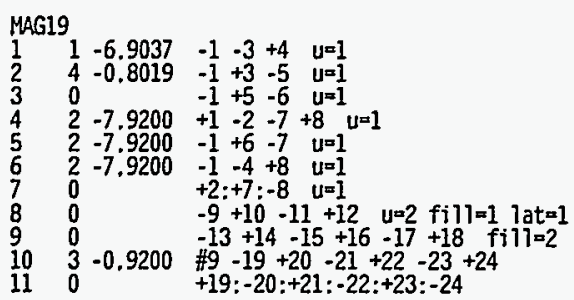

$\begin{array}{ll}1 & c z 6.76 \\ 2 & c z 6.79 \\ 3 & p z+5.27 \\ 4 & p z-12.60 \\ 5 & p z+5.80 \\ 6 & p z+12.58 \\ 7 & p z+12.63 \\ 8 & p z-12.63 \\ 9 & p x+8.573 \\ 10 & p x-8.573 \\ 11 & p y+8.573 \\ 12 & p y-8.573 \\ 13 & p x+26.258999 \\ 14 & p x-26.258999 \\ 15 & p y+26.258999 \\ 16 & p y-26.258999 \\ 17 & p z+12.78 \\ 18 & p z-12.78 \\ 19 & p x+41.259 \\ 20 & p x-41.259 \\ 21 & p y+41.259 \\ 22 & p y-41.259 \\ 23 & p z+27.78 \\ 24 & p z-27.78\end{array}$

mode $n$

Imp: 1 9r 0

ml $92234.50 \mathrm{c}$

$92234.50 \mathrm{c}$

$92235.50 \mathrm{c}$

$92238.50 \mathrm{c}$

$8016,50 \mathrm{c}$

6012.50 $1001.50 \mathrm{C}$

mtl lwtr.01t

$24000.50 \mathrm{c}$

25055 . $51 \mathrm{c}$

6000.50

$28000.50 \mathrm{c}$

$1.48591-4$

1. $48591-4$

3. $86922-5$

$8.26350-4$

$3.37790-2$

$6.89125-3$

$2.16009-2$

1.74286-2

$1.73633-3$

1.

5.72074-3
SCYLINDER OF URANTUM OIOXIDE WITH ALCOHOL SALCOHOL LAYER ABOVE OXIDE

SSTAINLESS STEEL CONTAINER

SSTAIMLESS STEEL (TOP)

SSTAINLESS STEEL (BOTTOM)

SVOID

SCELL FILLEO WITH 1-7

$\$ 3 \times 3 \times 1$ ARRAY

SPOLYETHYLENE REFLECTOR

SZERO IMPORTANCE OUTSIDE WORLD
Final Report

January 1996 


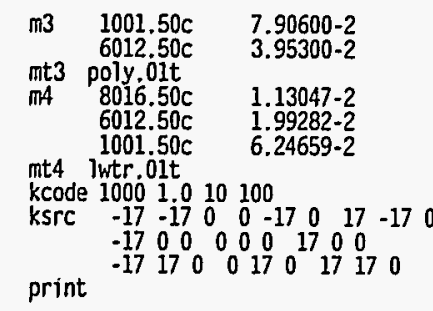

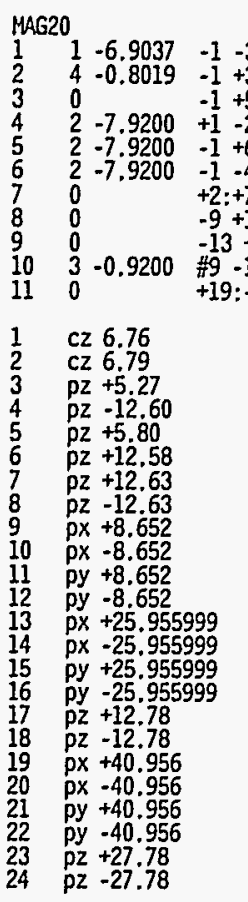

\section{mode $n$}

imp:n 1 gr 0

$92234.50 c$

$92235.50 \mathrm{c}$

$92236.50 \mathrm{c}$

$92238.50 \mathrm{c}$

$8016.50 \mathrm{c}$

$6012.50 \mathrm{c}$

mt1 iwtr.01t

$24000.50 \mathrm{c}$

$25055.51 \mathrm{c}$

26000.50

m3 $1001.50 \mathrm{c}$

$6012.50 \mathrm{c}$

$m+3$ poly.01t
$m 4 \quad 8016.50 \mathrm{c}$

$6012.50 \mathrm{c}$

$1001.50 \mathrm{c}$

mt4 lwtr.01t

$-1-3+4 \quad u=1$

$-1+3 \quad-5 \quad u=1$

$+1-2 \quad-7+8 \quad u=1$

$1+6 \quad-7 \quad u=1$

$\begin{array}{ll}-4+8 & u=1 \\ 2 \cdot+7 \cdot-8 & u=1\end{array}$

$9+10-11+12 \quad u=2$ fillel lat $=1$

$+14-15+16-17+18 \quad$ fill $=$ ?

$-19+20-21+22-23+24$

\$CYLINDER OF URANIUM DIOXIDE WITH ALCOHOL

SALCOHOL LAYER ABOVE OXIDE

SVOID

SSTAINLESS STEEL CONTAINER

SSTAINLESS STEEL (TOP)

SSTAINLESS STEEL (BOTTOM)

SVOID

SCELL FILLED WITH 1-7

\$3X3X1 ARRAY

SPOLYETHYLENE REFLECTOR

SZERO IMPORTANCE OUTSIDE WORLD

$1.48591-4$

1.39213-2

$8.26350-4$

3.37790-2

$6.89125-3$

$1.74286-2$

$1.73633-3$

$5.93579-2$

$7.00500-2$

$3.95300-2$

$13047-2$

99282-2

$.24659-2$

$\begin{array}{lrllllllllll}\text { kcode } & 1000 & 1.0 & 10 & 100 & & & & \\ \text { ksrc } & -17 & -17 & 0 & 0 & -17 & 0 & 17 & -17 & 0\end{array}$

$\begin{array}{llllllllll}-17 & 0 & 0 & 0 & 0 & 0 & 17 & 0 & 0\end{array}$

print

ROT1

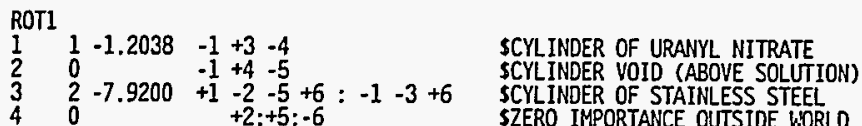

$1 \quad$ CZ 13.96

CZ 14.28 
$\begin{array}{ll}4 & p z+31.20 \\ 5 & p z+41.60 \\ 6 & p z-0.64\end{array}$

mode $n$

imp:n 11110

$92234.50 \mathrm{c}$

$92235.50 \mathrm{c}$

$92238.50 \mathrm{c}$

$7014.50 \mathrm{C}$

$1001.50 \mathrm{C}$

$\mathrm{mtl}$ lwtr.01t

$3.83099-6$

$3.47765-4$

$1.61304-6$

9. $23065-4$

.50450-2

6.32366-2

$24000.50 \mathrm{C}$

$5.93579-2$

kcode $10001.010100^{28000}$

print

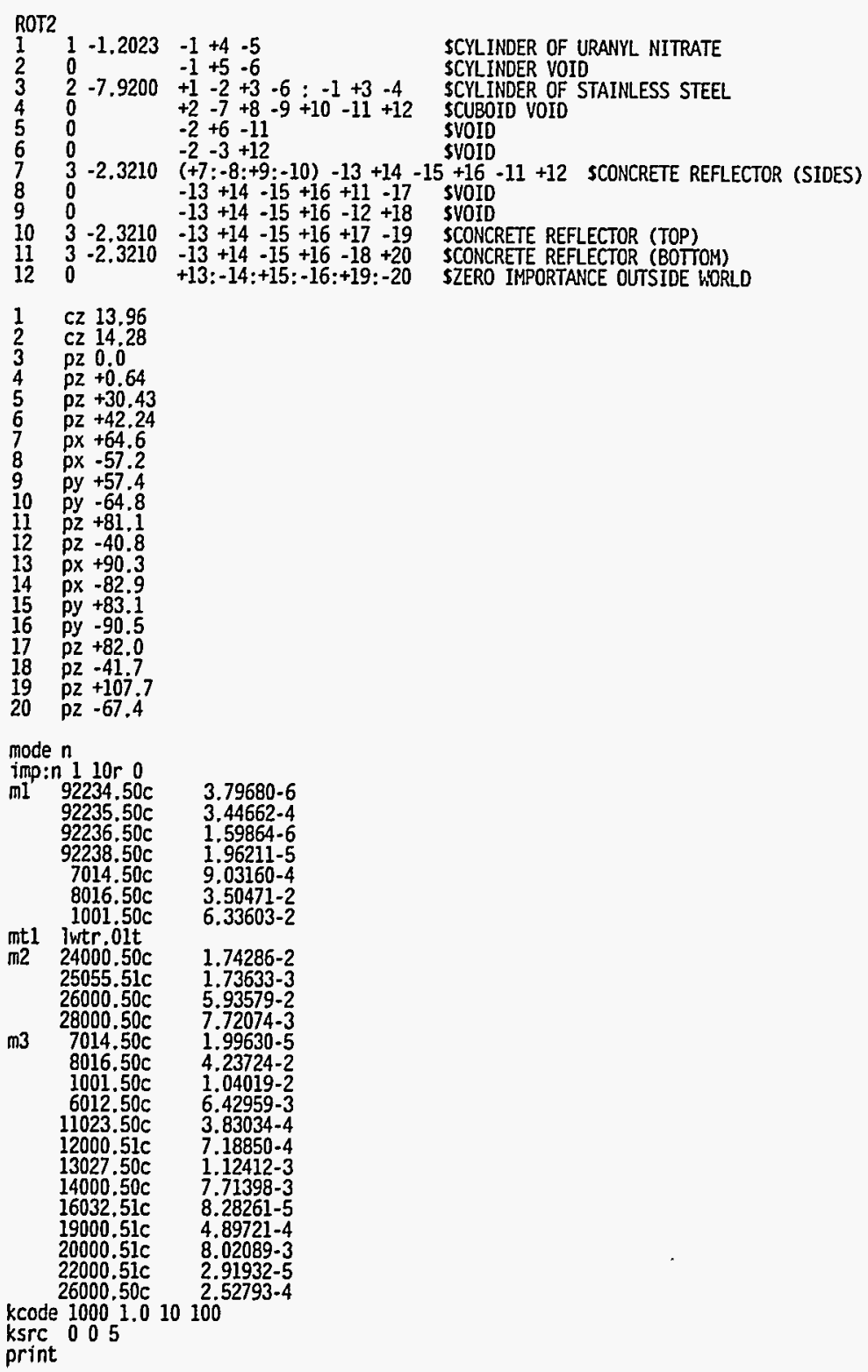


POEF-SH-33 


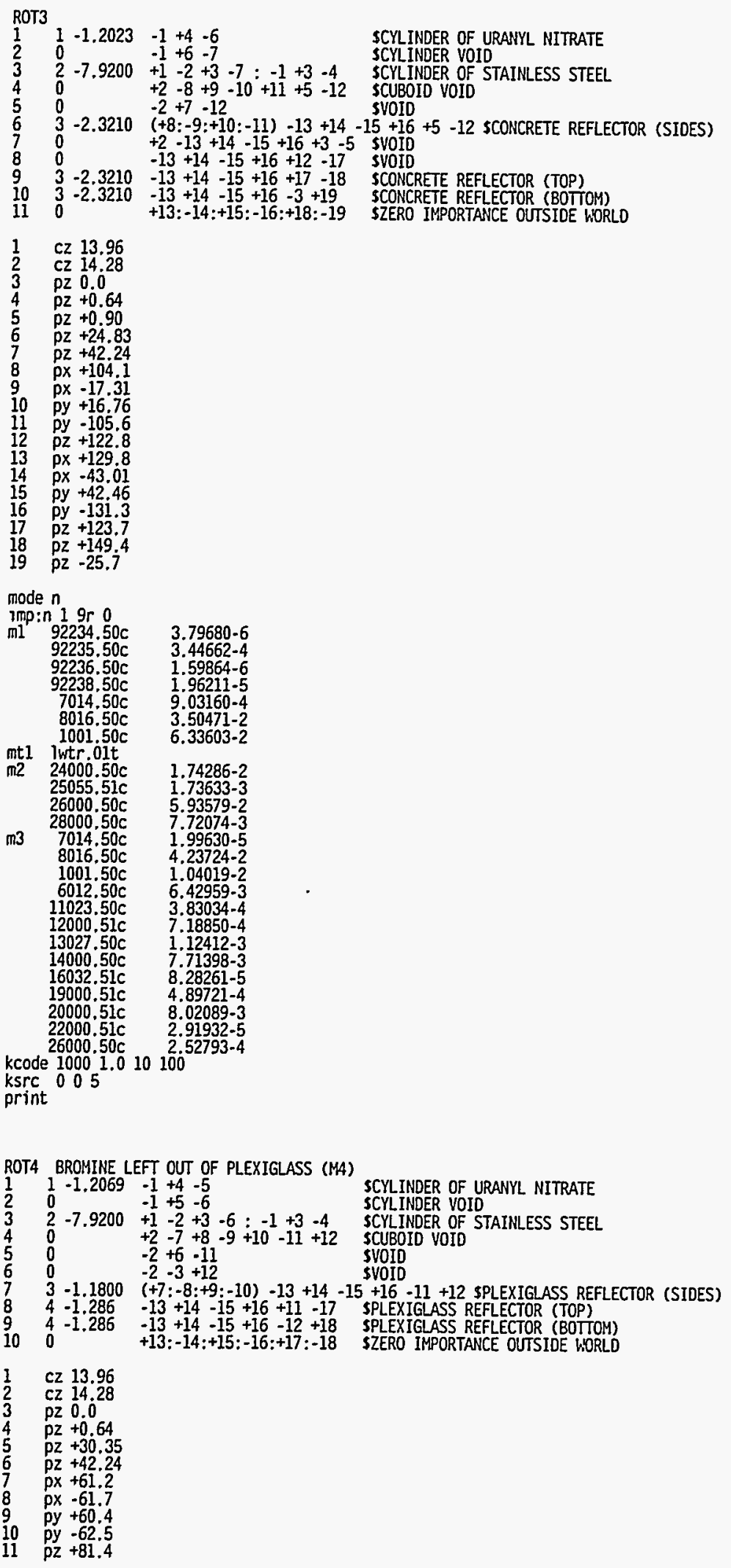

Final Report

January 1996 

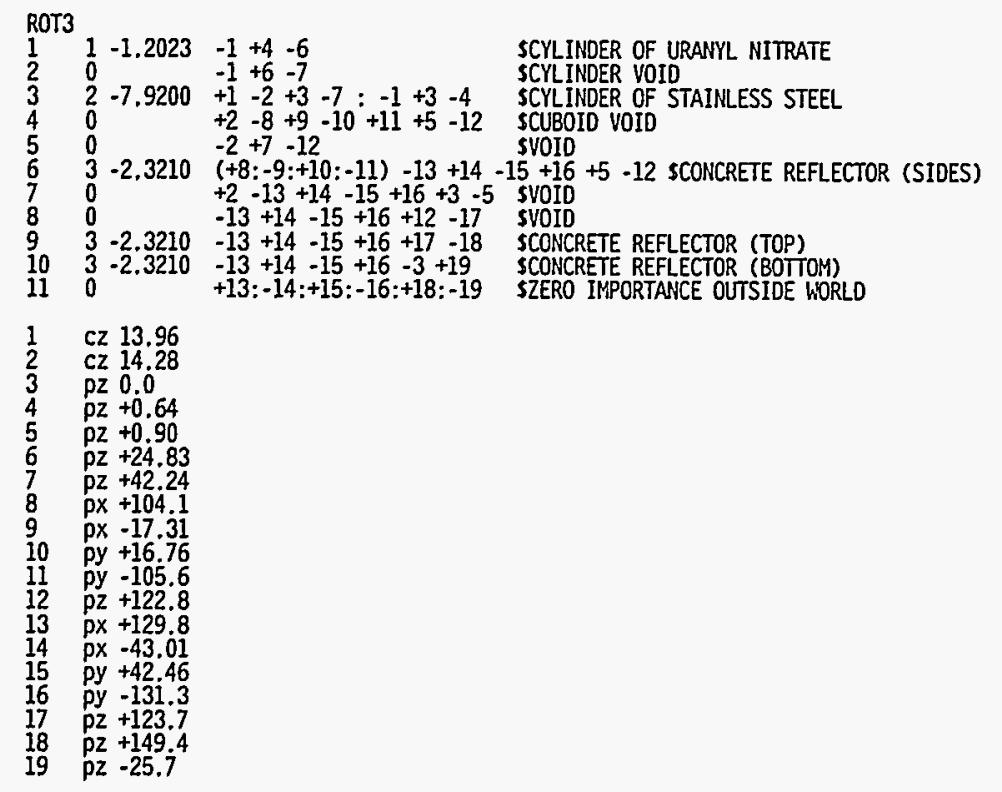

mode $n$

$$
\begin{aligned}
& \text { imp: n } 1 \text { gr } 0 \\
& \text { 92235.50 } \quad 3.79680-6 \\
& 92236.50 \mathrm{c} \quad 1.59864-6 \\
& \text { 92238.50c } 1.96211-5 \\
& 7014.50 \mathrm{c} \quad 9.03160 .4 \\
& 8016.50 \mathrm{C} \quad 3.50471-2 \\
& 11001.50 \mathrm{c} \\
& 6.33603-2 \\
& \text { mt1 lwtr.01t } \\
& 24000.50 \mathrm{c} \quad 1.74286-2 \\
& \text { 1.73633-3 } \\
& 26000.50 \mathrm{C} \quad 5.93579-2
\end{aligned}
$$

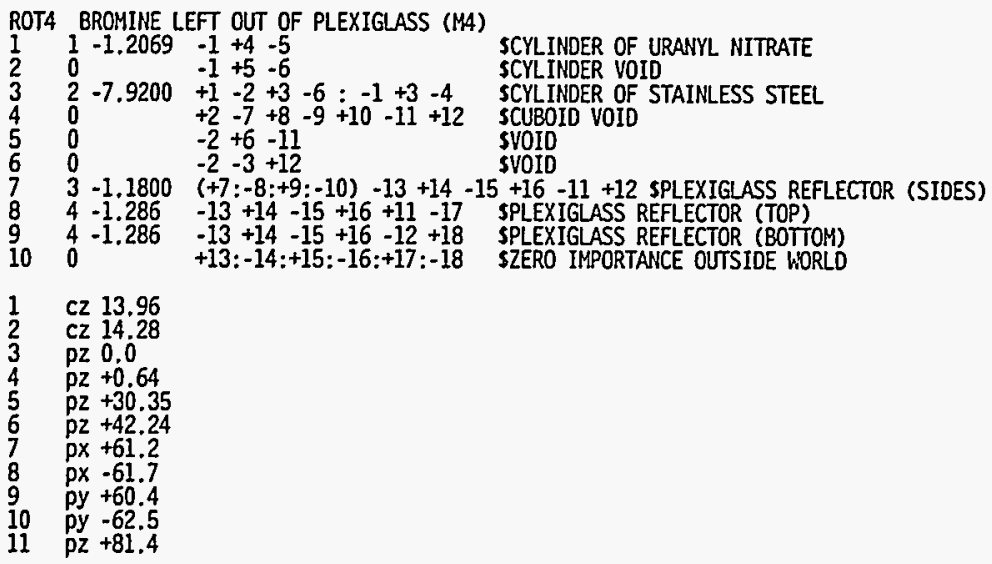

January 1996 
$\begin{array}{ll}12 & p z-41.5 \\ 13 & p x+82.0 \\ 14 & p x-82.5 \\ 15 & p y+81.2 \\ 16 & p y-83.3 \\ 17 & p z+101.6 \\ 18 & p z-62.3\end{array}$

mode $n$

imp: $n 18 r \quad 0$

3.88306-6

$92236.50 \mathrm{C}$

$92238.50 \mathrm{c}$

$7014.50 \mathrm{C}$

$3.52492-4$

$1.63496-6$

2.00669-5

$9.19354-4$

6.33067-2

mtl lwtr 01

$24000.50 \mathrm{c}$

$24000.50 \mathrm{c}$

$1.74286-2$

$5.93579-2$

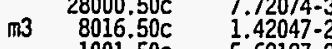

5.68187-2

$6012.50 \mathrm{c}$

$\pi 4 \quad 7014.50 \mathrm{c} \quad 5.5290 .5$

$8016.50 \mathrm{c} \quad 1.4231-2$

$1001.50 \mathrm{c} \quad 5.5166-2$

$6012.50 \mathrm{c} \quad 3.3966-2$

$15031.51 \mathrm{C} \quad 3.8504-4$

$17000.50 \mathrm{C} \quad 3.5605-4$

kcode 10001.010100

psrint

ROT5 BROMINE LEFT OUT OF PLEXIGLASS (HA)

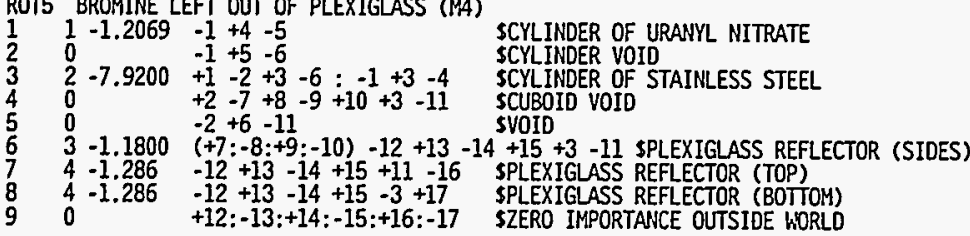

$\begin{array}{ll}1 & c z 13.96 \\ 2 & c z 14.28 \\ 3 & p z ~ 0.0 \\ 4 & p z+0.64 \\ 5 & p z+25.67 \\ 6 & p z+42.24 \\ 7 & p x+105.0 \\ 8 & p x-17.91 \\ 9 & p y+17.06 \\ 10 & p y-105.6 \\ 11 & p z+12.9 \\ 12 & p x+125.8 \\ 13 & p x-38.71 \\ 14 & p y+37.86 \\ 15 & p y-126.4 \\ 16 & p z+143.1 \\ 17 & p z-20.8\end{array}$

\section{mode $n$}

imp: $n 17 \mathrm{r} 0$

$92234.50 \mathrm{c}$

$92235.50 \mathrm{c}$

92236.50

$92238,50 \mathrm{c}$

$7014.50 \mathrm{c}$

$8016.50 \mathrm{C}$

$1001.50 \mathrm{c}$

mt1 liwtr. olt

$24000.50 \mathrm{c}$

$25055.51 \mathrm{c}$

$26000.50 \mathrm{c}$

$28000.50 \mathrm{c}$

$\mathrm{m} 3 \quad 8016.50 \mathrm{c}$

$1001.50 \mathrm{c}$

6012.50

$7014.50 \mathrm{c}$

$8016.50 \mathrm{c}$

$1001.50 \mathrm{c}$

$6012.50 \mathrm{C}$

$15031.51 \mathrm{C}$

:-13:+14:-15:+16:-17

IMPRTANCE OUTSIDE WORLO

kcode 10001.010100

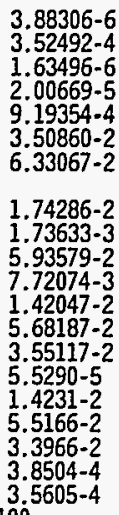

.

2.00669 .5

9.19354.4

$6.33067-2$

.74286-2

93579-2

.72074-3

.

$3.55117-2$

$5.5290-5$

. $5166-2$ 
ksrc 005

print

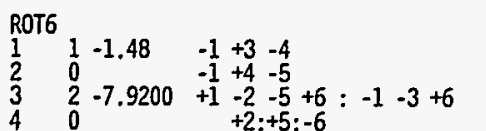

SCYLINDER OF URANYL NITRATE

SCYLINDER VOID (ABOVE SOLUTION)

SCYLINDER OF STAINLESS STEEL

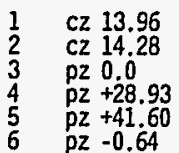

mode $n$

imp:n 111

$\mathrm{ml}$ 92234.50 $\quad 9.11806-6$

$92235.50 \mathrm{C} \quad 8.27710-4$

$92236.50 \mathrm{C} \quad 3.83915-6$

$92238.50 \mathrm{c}-4.71203-5$

$7014.50 \mathrm{C} \quad 2.10197-3$

$1001.50 \mathrm{C} \quad \mathbf{5} .81438-2$

mit lwtr oit

$24000.50 \mathrm{c} 1.74286-2$ 25055.51c $1.73633-3 \quad 26000.50 \mathrm{c} 5.93579-2$

$28000.50 \mathrm{c} 7.72074-3$

kcode 10001.010100

ksrc 005

print

$\begin{array}{lll}\text { ROT } & \\ 1 & 1 & -1.4636 \\ 2 & 0 & \\ 3 & 2 & -7.9200 \\ 4 & 0 & \\ 5 & 0 & \\ 6 & 3 & -2.3210 \\ 7 & 0 & \\ 8 & 0 & \\ 9 & 3 & -2.3210 \\ 10 & 3 & -2.3210 \\ 11 & 0 & \end{array}$

$\begin{array}{lll}-1+4 & -5 \\ -1 & +5 & -6\end{array}$

$+1-2+3-6:-1+3-4$

SCYLINDER OF URANYL NITRATE

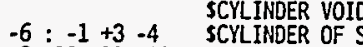

$-2+6-11:-2-3+12$ SVOID

$(+7:-8:+9:-10)-13+14-15+16-11+12$ SCONCRETE REFLECTOR (SIDES)

$-13+14-15+16+11-17$ 5VOID

-13+14 $-15+16+17-19$ SCONCRETE REFIECTOR (TOP)

$-13+14-15+16-18+20$ SCONCRETE REFLECTOR (BOTTOM)

$\begin{array}{ll}1 & c z 13.96 \\ 2 & c z 14.28 \\ 3 & p z 0.0 \\ 4 & p z+0.64 \\ 5 & p z+27.87 \\ 6 & p z+42.24 \\ 7 & p x+64.6 \\ 8 & p x-57.2 \\ 9 & p y+57.4 \\ 10 & p y-64.8 \\ 11 & p z+81.1 \\ 12 & p z-40.8 \\ 13 & p x+90.3 \\ 14 & p x-82.9 \\ 15 & p y+83.1 \\ 16 & p y-90.5 \\ 17 & p z+82.0 \\ 18 & p z-41.7 \\ 19 & p z+107.7 \\ 20 & p z-67.4\end{array}$

$+13:-14:+15:-16:+19:-20$ SZERO IMPORTANCE OUTSIDE WORLD

mode $n$

imp: 1 1 $9 \mathrm{r} 0$

$92235.50 \mathrm{c}$

$92235.50 \mathrm{C}$

$92238.50 \mathrm{C}$

$7014.50 \mathrm{c}$

$8016.50 \mathrm{c}$

$1001.50 \mathrm{c}$

mtl lwtr.0it

$24000.50 \mathrm{c}$

(25000.50

(2500.50

$\mathrm{m3}$

$28000.50 \mathrm{c}$

$8016.50 \mathrm{C}$

$1001.50 \mathrm{c}$

$6012.50 \mathrm{c}$

$11023.50 \mathrm{C}$

$12000.51 \mathrm{C}$

$13027.50 \mathrm{C}$

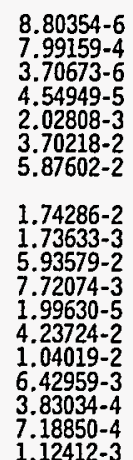

$8.80354-6$

$3.70673-6$

4.54949-5

$70218-2$

.87602-2

$4286-2$

(1)

$.72074-3$

$.99630-5$

$.04019-2$

$83034-4$

$1.12412-3$ 
$14000.50 \mathrm{C} \quad 7.71398-3$

$16032.51 \mathrm{C} \quad 8.28261-5$

$19000.51 \mathrm{C} \quad 4.89721-4$

$20000.51 \mathrm{c}$

$22000.51 \mathrm{C} \quad 2.91932-5$

$2600050 \mathrm{c} 252793-4$

kcode 10001.010100

ksre 005

print

Final Report

January 1996 

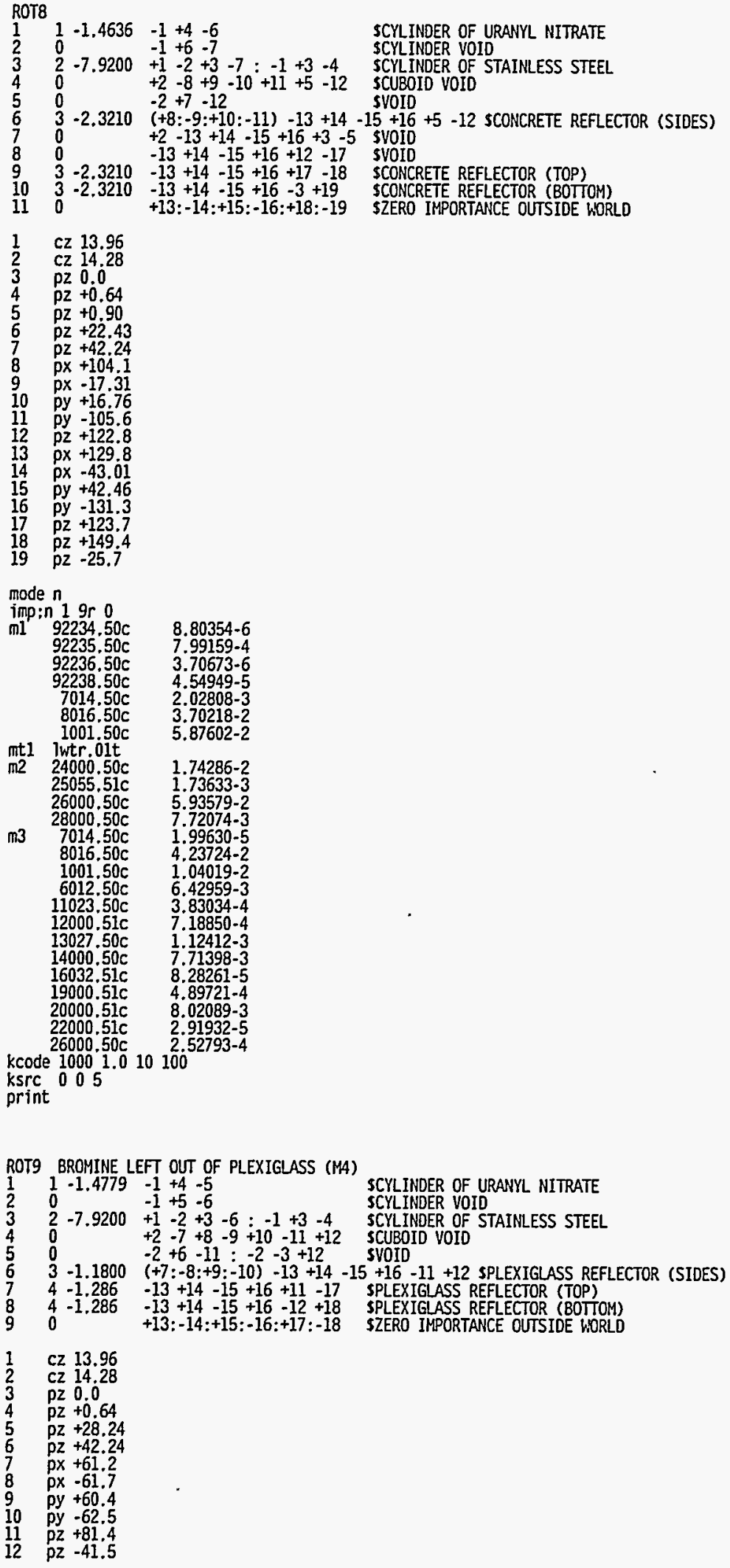
$\begin{array}{ll}13 & p x+82.0 \\ 14 & p x-82.5 \\ 15 & p y+81.2 \\ 16 & p y-83.3 \\ 17 & p z+101.6 \\ 18 & p z-62.3\end{array}$

mode $n$

imp: $n 17 r$

$\mathrm{ml}$ 92234.50c

$92235.50 \mathrm{c}$

$92236,50 \mathrm{c}$

$92238.50 c$

$7014.50 \mathrm{c}$

$800.50 \mathrm{c}$

mt1 lwtr.01t

5.84951-2

9.08124-6

$8.24367-4$

$3.82365-6$

4.69300-5

$2.08999-3$

m2

$24000.50 \mathrm{c}$

$26000.50 \mathrm{C}$

1.74286-2

$1.73633-3$

5. $73579-2$

7.72074-3

$28000.50 \mathrm{c}$

1001.50

$6012.50 \mathrm{C}$

$\mathrm{m4} \quad 7014.50 \mathrm{c}$

$8016.50 \mathrm{c}$

$1001.50 \mathrm{c}$

$6012.50 \mathrm{C}$

$15031.51 \mathrm{C}$

$5.68187-2$

3.55117-2

$5.5290-5$

$1.4231-2$

$3.3966-2$

. $8504-4$

$3.5605-4$

kcode 10001.010100

ksrc 005

print

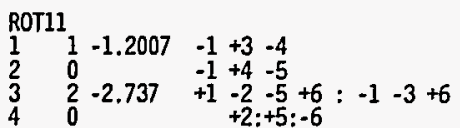

SCYLINDER OF URANYL NITRATE

SCYLINDER VOID

SCYLINDER OF ALUMIMUM

SZERO IMPORTANCE OUTSIDE WORLO $\begin{array}{ll}1 & \mathrm{cz} 14.005 \\ 2 & \mathrm{cz} 14.325 \\ 3 & \mathrm{pz} 0.0 \\ 4 & \mathrm{pz}+33.55 \\ 5 & \mathrm{pz}+41.9 \\ 6 & \mathrm{pz}-0.64\end{array}$

mode $n$

imp: $1 \frac{1}{9} 11100$

$92235.50 \mathrm{c}$

$92236.50 \mathrm{c}$

$7014.50 \mathrm{c}$

(2)

100150

mt1 lwtr.01t

$12000.51 \mathrm{c}$

$13027.50 \mathrm{C}$

2000.51

$24000.50 \mathrm{C}$

$25055.51 \mathrm{c}$

$26000.50 \mathrm{C}$

kcode 10001010100

ksrc 005

ksrc
print

$$
\begin{aligned}
& 3.75841-6 \\
& 3.41177-4 \\
& 1.58248-6 \\
& 1.94227-6 \\
& 9.02307-4 \\
& 3.50416-2 \\
& 6.33760-2 \\
& 6.78153-4 \\
& 5.95056-2 \\
& 3.52126-4 \\
& 1.0327-5 \\
& 5.38898-5 \\
& 2.10015-5 \\
& 1.38721-4 \\
& 6.42881-5
\end{aligned}
$$

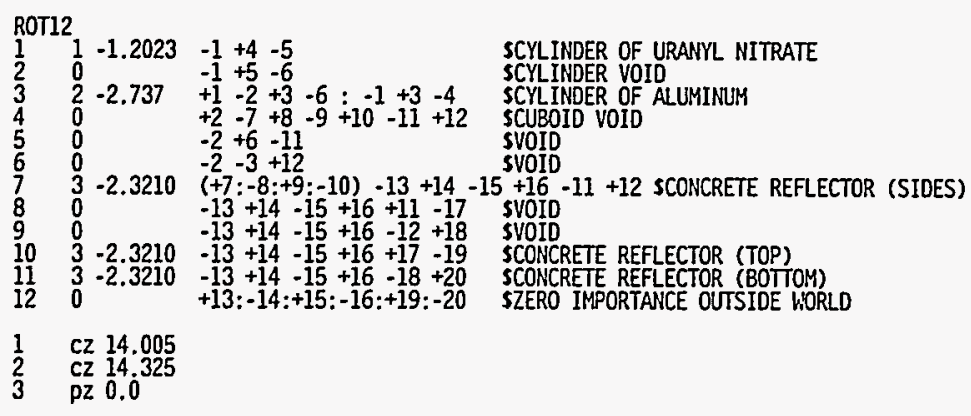


mode $n$

imp: $n 1$ 10r 0 $2234.50 \mathrm{C}$ $92235.50 \mathrm{c}$ $92238.50 \mathrm{C}$ $7014.50 \mathrm{c}$ Dib. $50 \mathrm{C}$

3.79680-6

$3.44662-4$

$1.59864-6$

$.03160-4$

6.33603-2

$\mathrm{m} 2$

iwtr.oit

6.78153-4

5. $52126-4$

$1.03277-5$

2000.51c

$24000.50 \mathrm{c}$

5.38898

2. $10015-5$

m3

6.42881-5

$7014.50 \mathrm{C} \quad 1.99630-5$

$1.04019-2$

$6.42959-3$

$3.83034-4$

$7.18850-4$

$1.12412 \cdot 3$

$8.72861-5$

$4.89721-4$

$4.0201-3$

8.02089 -

kcode 10001.010100

ksrc
print

$\begin{array}{lll}\text { ROT13 } & \\ 1 & 1 & -1.2023 \\ 2 & 0 & \\ 3 & 2 & -2.737 \\ 4 & 0 & \\ 5 & 0 & \\ 5 & 0 & \\ 6 & 3 & -2.3210 \\ 7 & 0 & \\ 8 & 0 & \\ 9 & 3 & -2.3210 \\ 10 & 3 & -2.3210 \\ 11 & 0 & \end{array}$

$\begin{array}{rrr}-1+4 & -6 \\ -1+6 & -7\end{array}$

$+1-2+3-7 \cdot-1+3-4$

$+2-8+9-10^{\circ}+11+5-12$ $-2+7-12$

SCYLINDER OF URANYL NITRATE

SCYLINDER VOIO

CYLINDER OF ALUMINUM

CUBOID VOID

$+2-13+14-15+16+3-5$ SVOID

$-13+14-15+16+17-18$ SCONCRETE REFLECTOR (TOP)

$-13+14-15+16-3+19$ SCOMCRETE REFECTOR (BOTOM)

$110+13:-14:+15:-16:+18:-19$ SZERO IMPORTANCE OUTSIDE WORLD

$\begin{array}{ll}1 & c z 14.005 \\ 2 & c z 14.325 \\ 3 & p z ~ 0.0 \\ 4 & p z+0.64 \\ 5 & p z+0.90 \\ 6 & p z+25.34 \\ 7 & p z+42.54 \\ 8 & p x+104.1 \\ 9 & p x-17.8 \\ 10 & p y+16.5 \\ 11 & p y-105.6 \\ 12 & p z+122.8 \\ 13 & p x+129.8 \\ 14 & p x-43.5 \\ 15 & p y+42.2 \\ 16 & p y-131.3 \\ 17 & p z+123.7 \\ 18 & p z+149.4 \\ 19 & p z-25.7\end{array}$

mode $n$

imp: ${ }_{92} 19 r \quad 0$

92236.50c

$3.79680-6$

$3.44652-4$

$1.59864-6$

January 1996

Final Report

Validation of MCNP4A on the Battelle

PSRM IBM RS/6000 Workstation

Page 52 


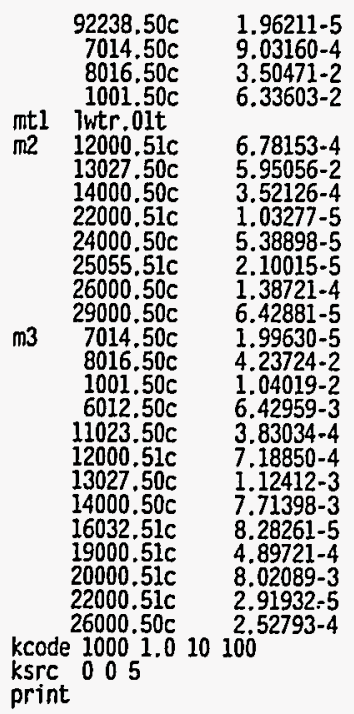

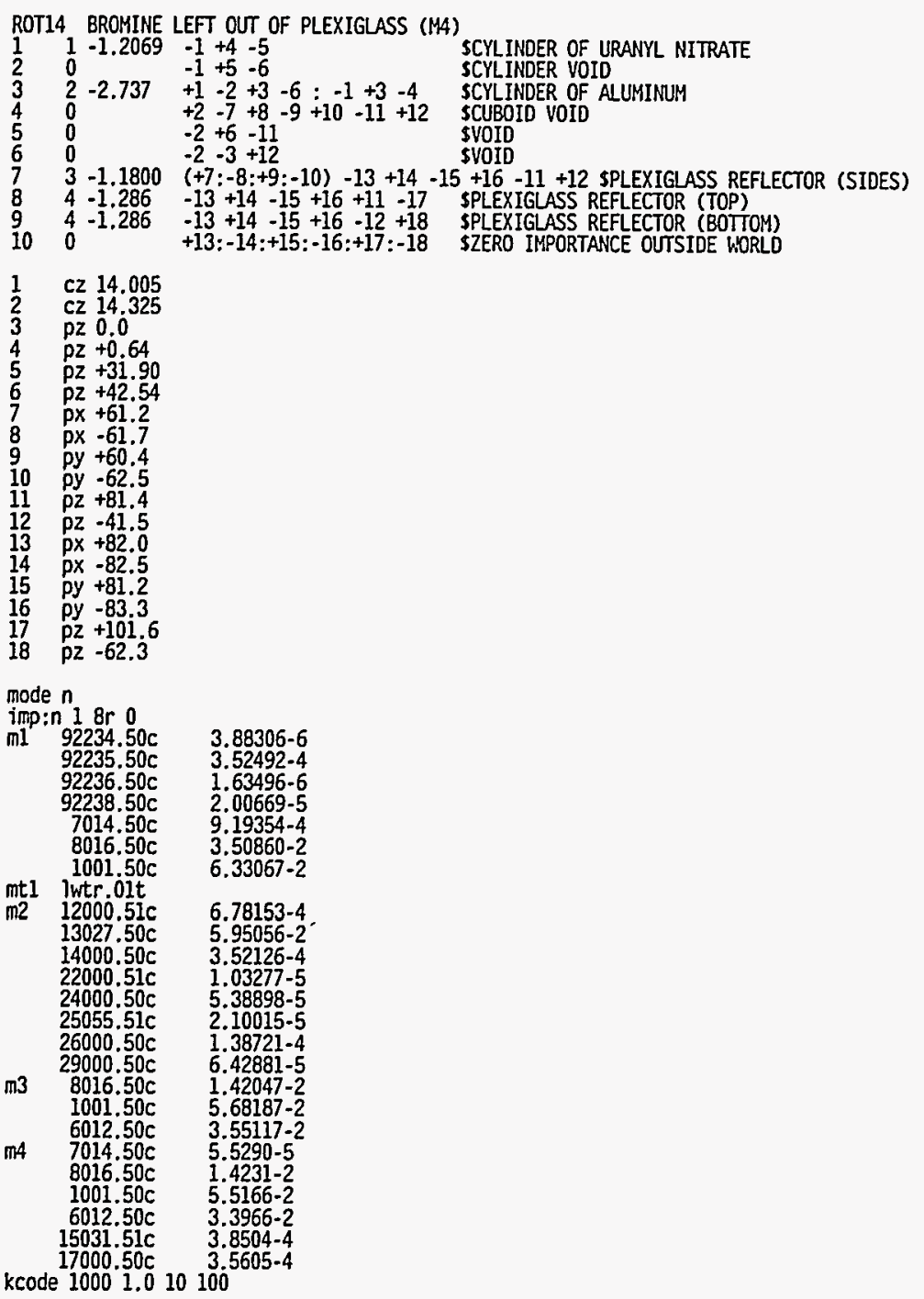

kcode 10001.010100 


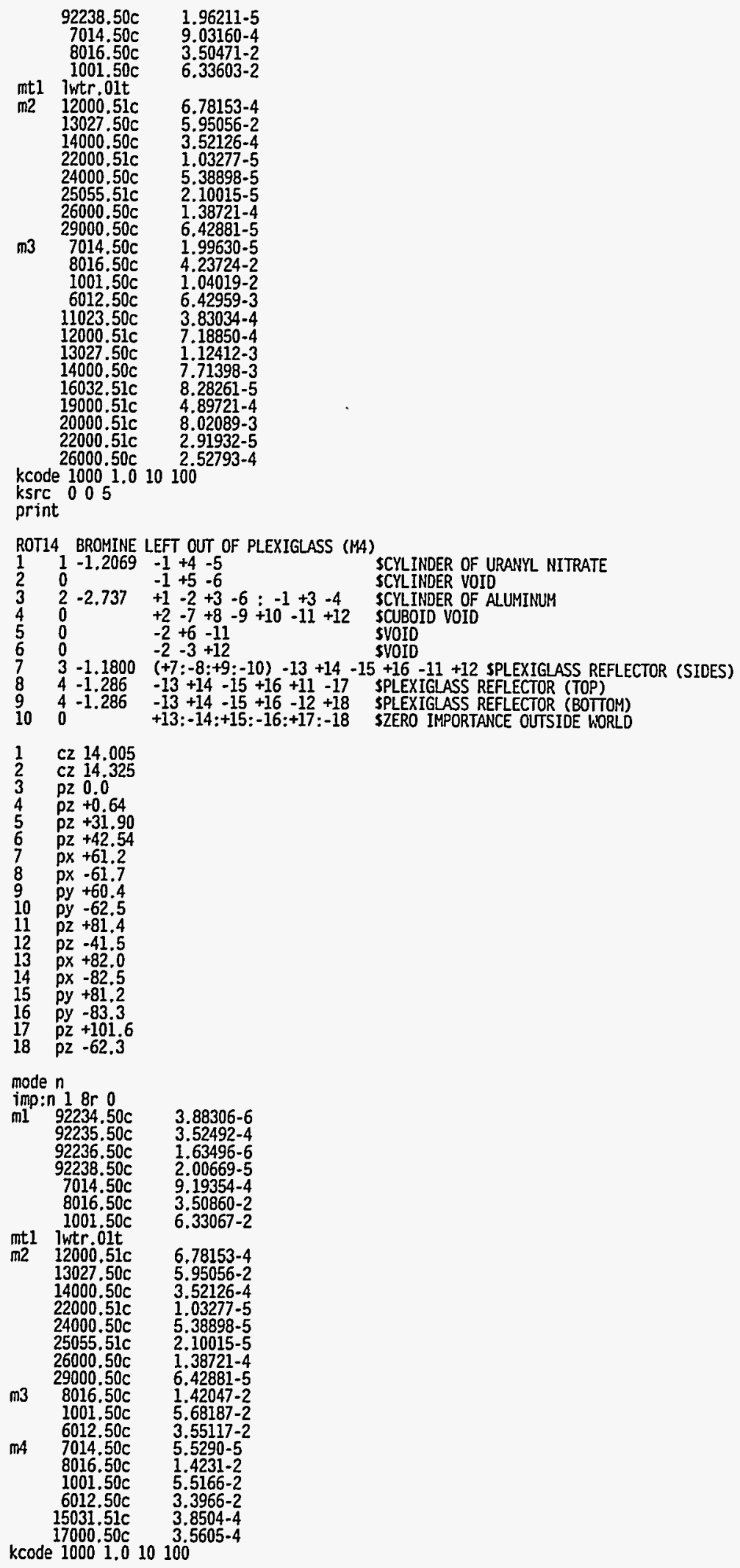

\begin{tabular}{lc} 
Final Report \\
January 1996 & Validation of MCNP4A on the Battelle \\
& PSRM IBM RS/6000 Workstation \\
\hline
\end{tabular}


ksrc 005

print

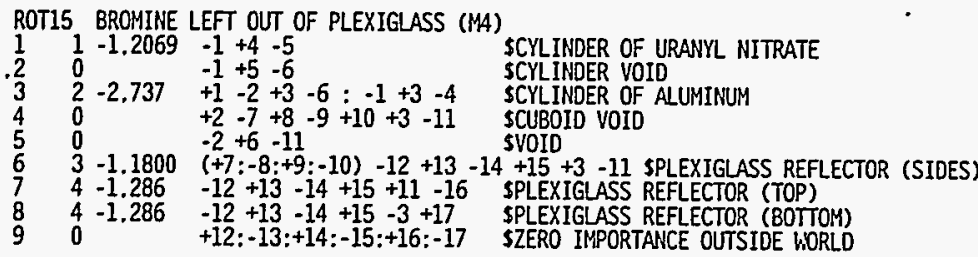

$\begin{array}{ll}1 & c z 14.005 \\ 2 & c z 14.325 \\ 3 & p z 0.0 \\ 4 & p z+0.64 \\ 5 & p z+25.90 \\ 6 & p z+42.54 \\ 7 & p x+105.0 \\ 8 & p x-17.95 \\ 9 & p y+17.52 \\ 10 & p y-105.6 \\ 11 & p z+122.9 \\ 12 & p x+125.8 \\ 13 & p x-38.75 \\ 14 & p y+38.32 \\ 15 & p y-126.4 \\ 16 & p z+143.1 \\ 17 & p z-20.8\end{array}$

\section{mode $n$}

$i_{m 1}: n$

n $17 r 0$

$92234.50 \mathrm{C} \quad 3.88306-6$

$\begin{array}{ll}92235.50 \mathrm{C} & 3.52492-4\end{array}$

92236.50c $\quad 1.63496-6$

$701450 \mathrm{C} 2.00669-5$

$3014.50 \mathrm{c} \quad \mathrm{3} .5354-4$

$\begin{array}{ll}8016.50 \mathrm{C} & 3.50860-2 \\ 1001.50 \mathrm{C} & 6.33067-2\end{array}$

mt1 iwtr.01

iwtrolt $\quad 6.78153-4$

$13027.50 \mathrm{c}$

$14000.50 \mathrm{C}$

$22000.51 \mathrm{c}$

$24000,50 \mathrm{C}$

25055.51c

$26000.50 \mathrm{c}$

$29000.50 \mathrm{c}$

m3 $8016.50 \mathrm{c}$

$1001.50 \mathrm{c}$

$6012.50 \mathrm{c}$

m4 $7014.50 \mathrm{C}$

$8016.50 \mathrm{C}$

1001.50

$6012.50 \mathrm{c}$

$15031.51 \mathrm{c}$

$5.95056-2$

3. $52126-4$

$1.03277-5$

5.38898-5

2.10015-5

$6.3281-5$

. $42881-5$

$5.68187-2$

$3.68187-2$

5.5290 .5

$1.4231-2$

$5.5166-2$

$3.3966-2$

3.5605 .4

kcode 10001.010100

ksrc 005

print

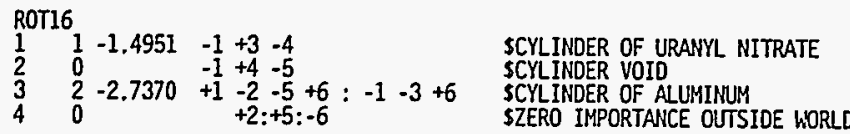

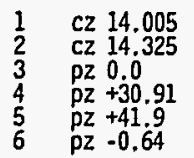

mode $n$

imp: $n 11 \frac{1}{1} 100$ $92235.50 \mathrm{c}$ $92236.50 \mathrm{c}$ $7014.50 \mathrm{c}$ $8016.50 \mathrm{C}$ $100150 \mathrm{c}$

mt1 lwtr.01t

9.40580-6

$8.53921-4$

3. $96073-6$

$4.86125-5$

2.16242-3

3.72609-2

5.82143-2

6.78153-4 


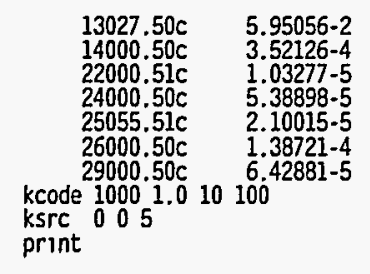

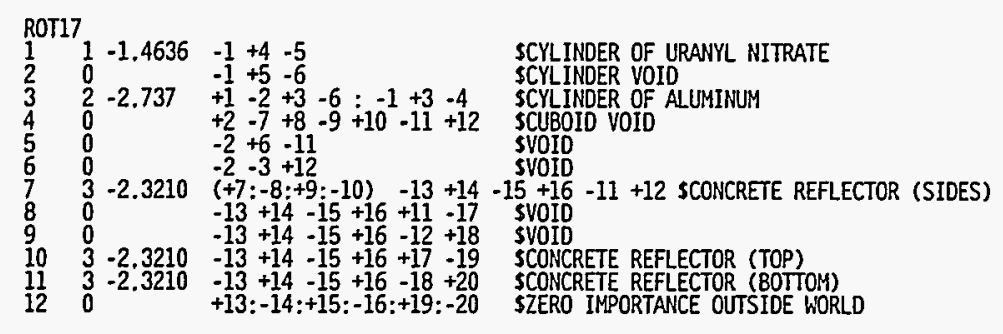

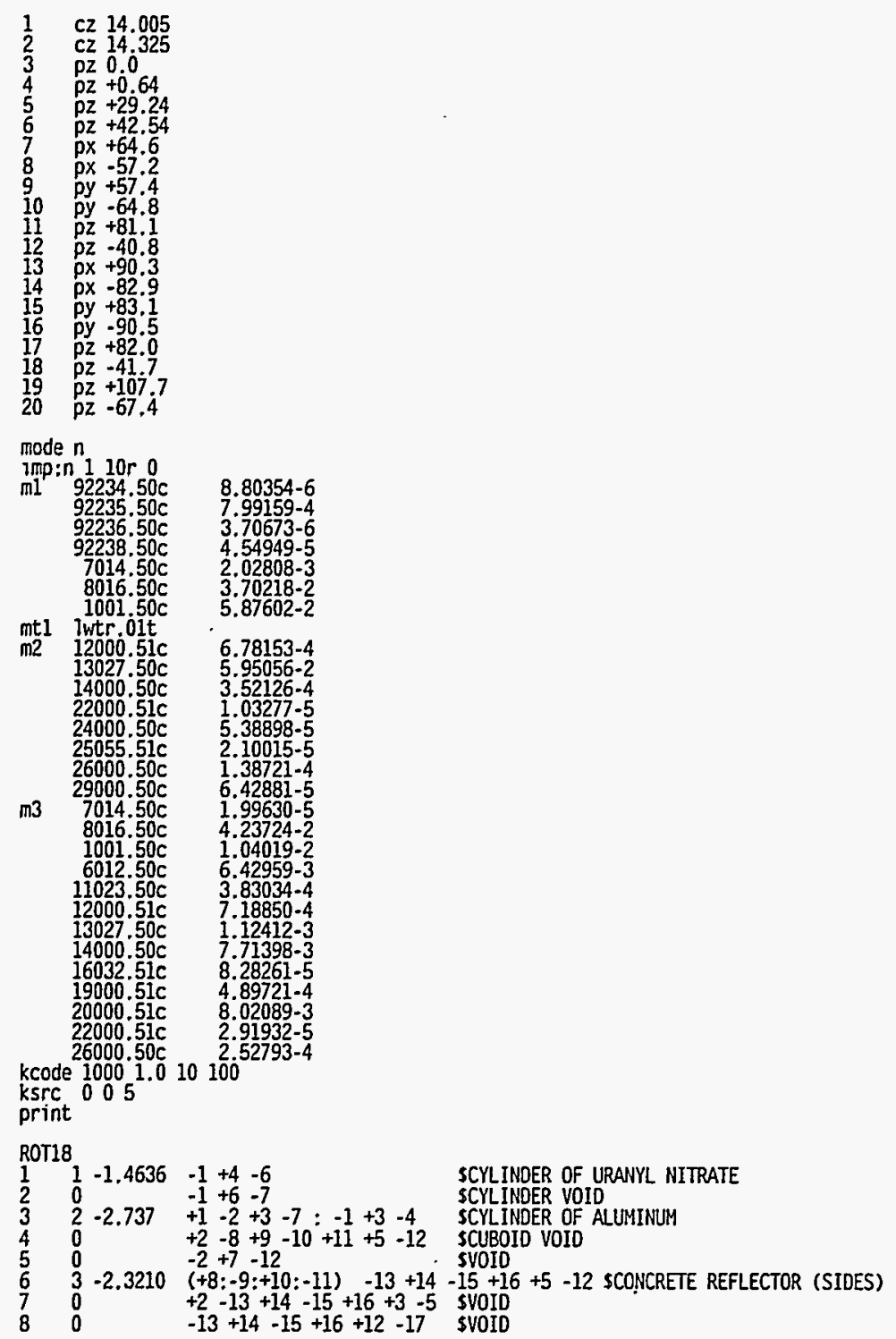

\begin{tabular}{cc} 
Final Report \\
January 1996 & Validation of MCNP4A on the Battelle \\
& PSRM IBM RS/6000 Workstation \\
\hline
\end{tabular}



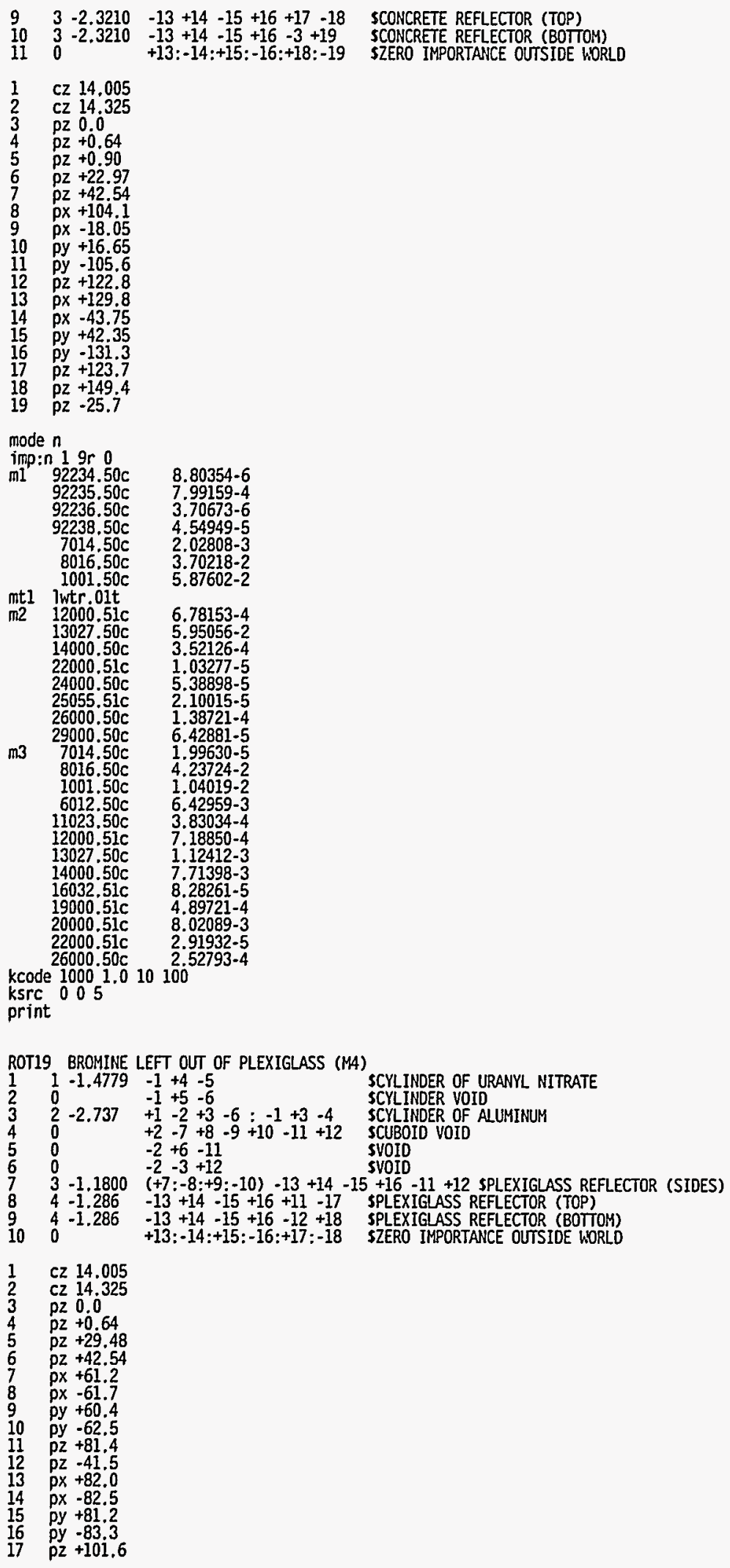

$-13+14-15+16+17-18$
$-13+14-15+16-3+19$

SCONCRETE REFLECTOR (TOP) $+13:-14:+15:-16:+18:-19$ SZERO IMPORTANCE OUTSIDE WORLD

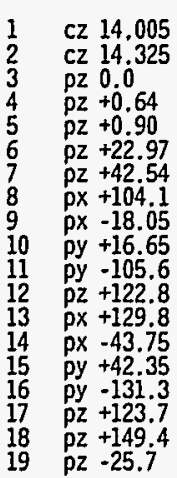

mode $n$

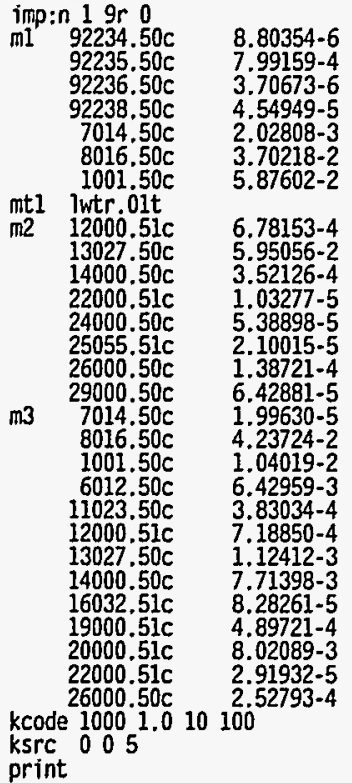


$\begin{array}{lll}18 & p z & -62.3\end{array}$

mode $n$

imp: $n 1$ 8r 0

$\mathrm{ml} 92234.50 \mathrm{c}$

9.08124-6

$92236.50 \mathrm{c} \quad 8.24367-4$

$92238.50 \mathrm{c} \quad 4.69300-5$

$7014.50 \mathrm{c} \quad 2.08999-3$

$016.50 \mathrm{c} 2.08951-3$

mt1 lwtr.01t $5.84951-2$

$\mathrm{m} 2 \quad 12000.51 \mathrm{c} \quad 6.78153 .4$

$13027.50 \mathrm{c} \quad 5.95056-2$

$14000.50 \mathrm{c} \quad 3.52126-4$

$22000.51 \mathrm{c} \quad 1.03277-5$

$24000.50 \mathrm{C} \quad 5.38898-5$

25055.51c 2.10015-5

$26000.50 \mathrm{c} \quad 1.38721-4$

$\mathrm{m}^{3} \quad 2900.50 \mathrm{c}$

$8016.50 \mathrm{c} \quad 6.42881-5$

6012.50

$1.42047-2$

5. $68187-2$
$3.55117-2$

$7014.50 \mathrm{C} \quad 5.5290-5$

$8016.50 \mathrm{C} \quad 1.4231-2$

$1001.50 \mathrm{c} \quad 5.5166-2$

$6012.50 \mathrm{c} \quad 3.3966-2$

$15031.51 \mathrm{c} \quad 3.8504-4$

kcode 10001.010100

ksrc 005

print

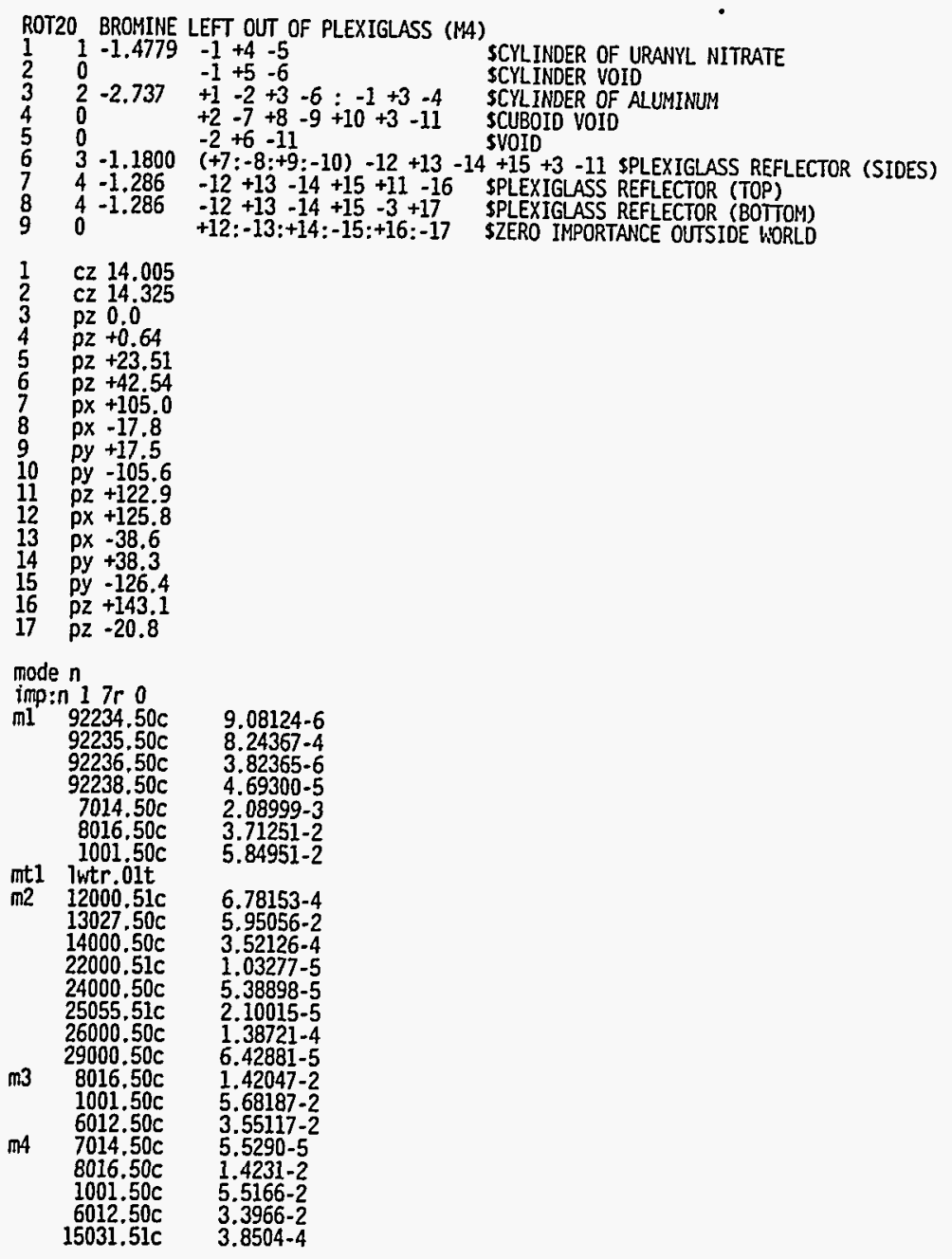




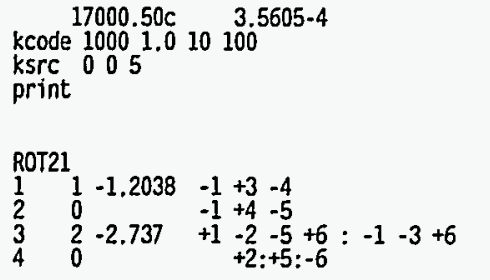

1 CZ 16.505

CZ 16.825

pz 0.0

$\mathrm{pz}+23.67$

$\mathrm{pz}+49.5$

\section{mode $n$}

imp: ${ }^{1} 1110$

ml $\begin{array}{ll}\text { imp: } 12234.50 \mathrm{c} & 3.83099-6 \\ 92235.50 \mathrm{c} & 3.47765-4\end{array}$

92236.50c $1.61304-6$

92238.50 C $1.97978-5$

$7014.50 \mathrm{C} \quad 9.23065-4$

$\begin{array}{ll}8016.50 \mathrm{C} & 3.50450-2 \\ 1001.50 \mathrm{c} & 6.32366-2\end{array}$

$1001.50 \mathrm{c}$

intr.01t

$\mathrm{m} 2$

$12000.51 \mathrm{c}$

$13027.50 \mathrm{c}$

5. $95056-2$

$\begin{array}{ll} & \\ & 1.03277-5\end{array}$

$24000.50 \mathrm{C} \quad 5.38898-5$

$25055.51 \mathrm{c} \quad 2.10015-5$

$26000.50 \mathrm{c} \quad 1.38721-4$

kcode 10001.010100

ksre 005

print

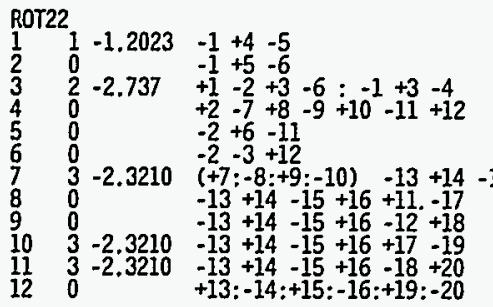

SCYLINOER OF URANYL NITRATE

SCYLINDER VOID

SCYLINDER OF ALUMINUM

促OID VOID

SVID

svot

OF URANYL NITRATE

STINDER VOID

R OF ALUMINUM

SZERO IMPORTANCE OUTSIDE KORLD $\begin{array}{ll}1 & c z 16.505 \\ 2 & c z 16.825 \\ 3 & p z \\ 4 & 0.0 \\ 4 & p z+0.64 \\ 5 & p z+23.49 \\ 6 & p z+50.14 \\ 7 & p x+64.6 \\ 8 & p x-57.2 \\ 9 & p y+57.4 \\ 10 & p y-64.8 \\ 11 & p z+81.1 \\ 12 & p z-40.8 \\ 13 & p x+90.3 \\ 14 & p x-82.9 \\ 15 & p y+83.1 \\ 16 & p y-90.5 \\ 17 & p z+82.0 \\ 18 & p z-41.7 \\ 19 & p z+107.7 \\ 20 & p z-67.4\end{array}$

mode $n$

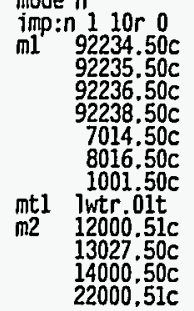

$3.79680-6$

$3.44662-4$

$1.59864-6$

$1.96211-5$

$9.03160-4$

$6.33603-2$

$6.78153-4$

$5.78553-4$

$3.52126-4$
$1.03277-5$

January 1996

Final Report

Validation of MCNP4A on the Battelle PSRM IBM RS/6000 Workstation 

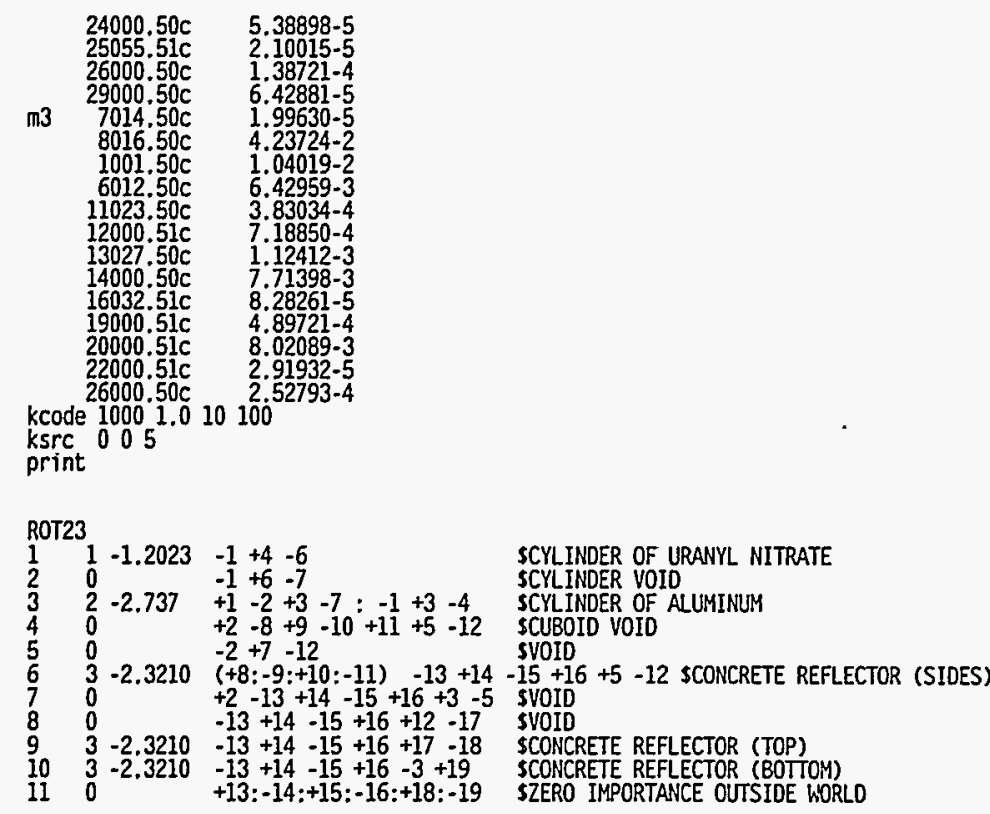

$\begin{array}{ll}1 & c z 16.505 \\ 2 & c z 16.825 \\ 3 & p z 0.0 \\ 4 & p z+0.64 \\ 5 & p z+0.90 \\ 6 & p z+18.88 \\ 7 & p z+50.14 \\ 8 & p x+104.1 \\ 9 & p x-17.92 \\ 10 & p y+16.825 \\ 11 & p y-105.6 \\ 12 & p z+122.8 \\ 13 & p x+129.8 \\ 14 & p x-43.62 \\ 15 & p y+42.525 \\ 16 & p y-131.3 \\ 17 & p z+123.7 \\ 18 & p z+149.4 \\ 19 & p z-25.7\end{array}$

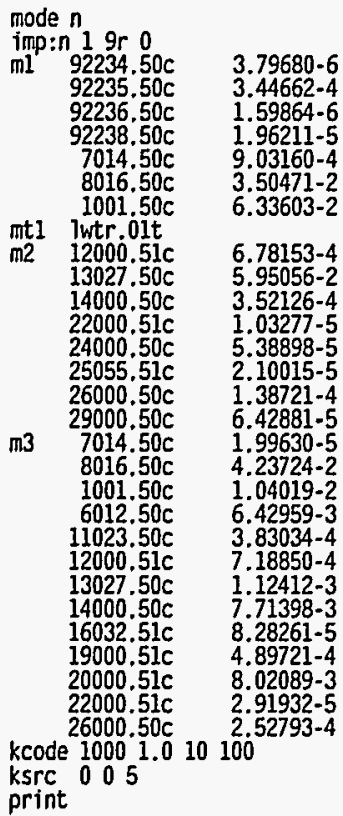

print 


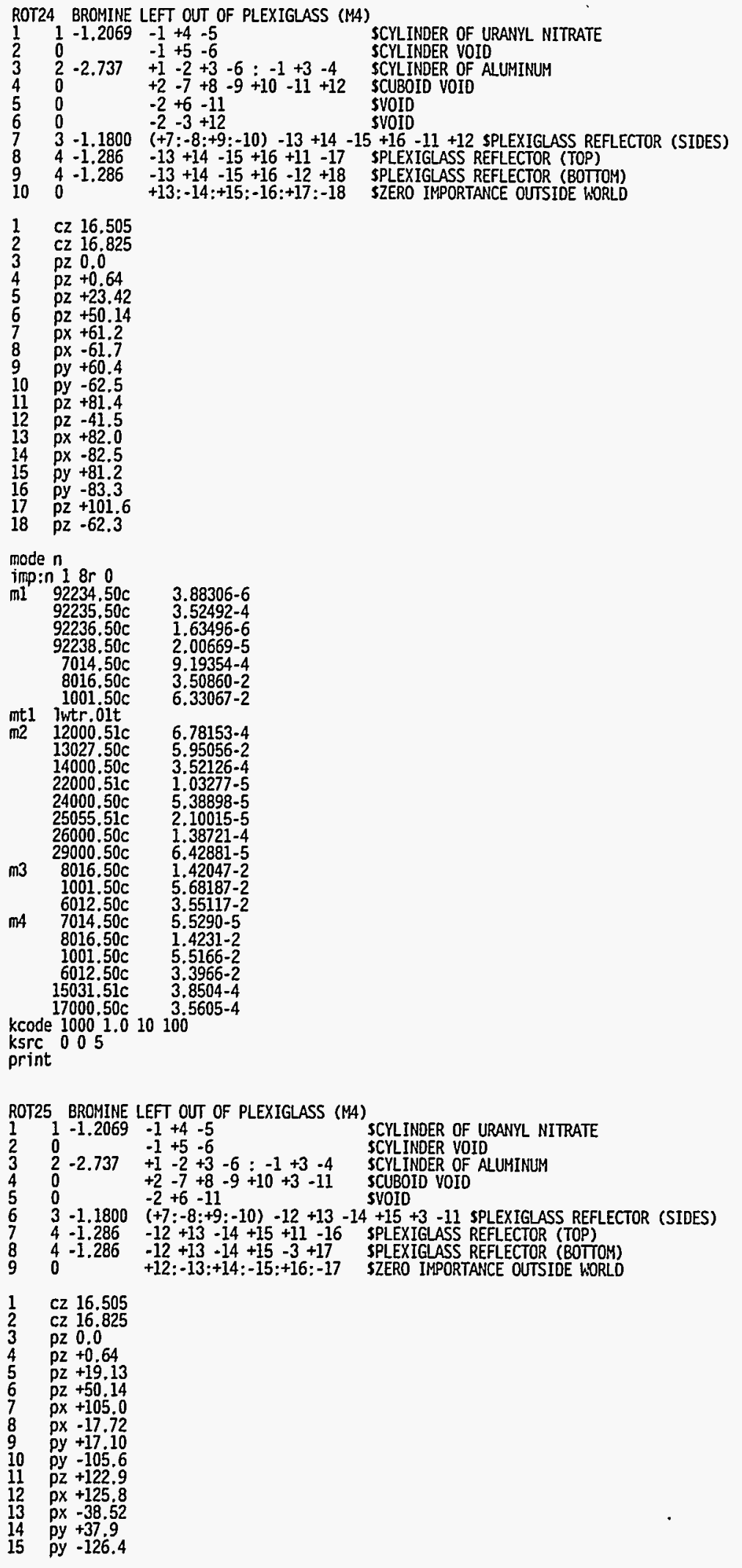




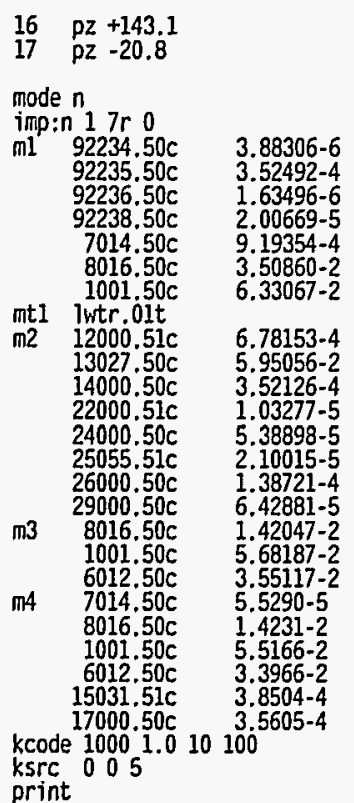

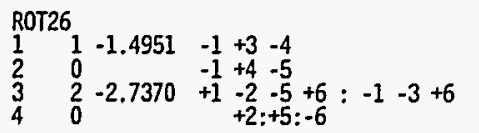

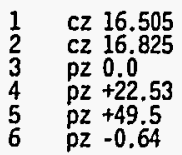

\section{mode $n$}

imp: n 111110

$\mathrm{ml} 92234.50 \mathrm{c}$ $92235.50 \mathrm{c}$ $92238.50 \mathrm{c}$ $7014.50 \mathrm{C}$ $8016.50 \mathrm{c}$ $1001.50 \mathrm{c}$

mt1 lwtr.01t $12000.51 \mathrm{c}$ $14000.50 \mathrm{c}$ $22000.51 \mathrm{c}$

$24000.50 \mathrm{c}$

$25055.51 \mathrm{c}$

$25055.51 \mathrm{c}$

$29000.50 \mathrm{c}$

kcode 10001.010100

ksrc 005

$9.40680-6$

$8.53921-4$

3.96073-6

4.86125-5

$2.16242-3$

5.72609-2

$6.78153-4$

$5.95056-2$

$3.52126-4$

5. $38898-5$

5. 38098.5

..10015-5 $6.42881-5$

print

$\begin{array}{lll}\text { ROT27 } & \\ 1 & 1 & -1.4636 \\ 2 & 0 & \\ 3 & 2 & -2.737 \\ 4 & 0 & \\ 5 & 0 & \\ 6 & 0 & \\ 7 & 3 & -2.3210 \\ 8 & 0 & \\ 9 & 0 & \\ 10 & 3 & -2.3210 \\ 11 & 3 & -2.3210 \\ 12 & 0 & \end{array}$

$\begin{array}{lll}-1+4 & -5 \\ -1+5 & -6\end{array}$

$+1-2+3-6=-1+3-4$

$+2-7+8-9+10-11+12$ $-2+6-11$

(2) -12

(SIDES)

SCYL INDER OF URANYL NITRATE

SCYLINDER VOID

ULMINUY

SZERO IMPORTANCE OUTSIDE WORLD

\footnotetext{
$\begin{array}{lll}\frac{1}{2} & \mathrm{CZ} & 16.505 \\ 2 & \mathrm{CZ} & 16.825\end{array}$

$\begin{array}{lll}2 & c z & 16.825 \\ 3 & p z & 0.0\end{array}$

$\mathrm{pz}+0.64$
}

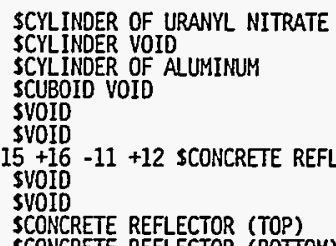

\section{Final Report}

January 1996 


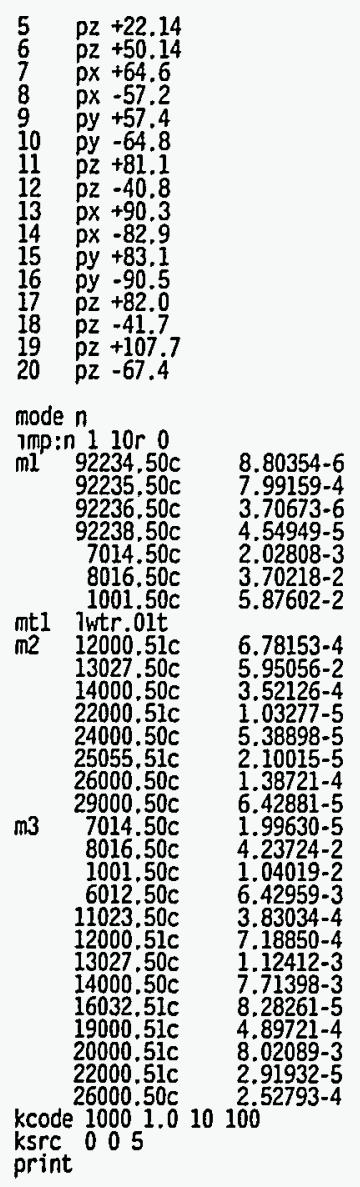




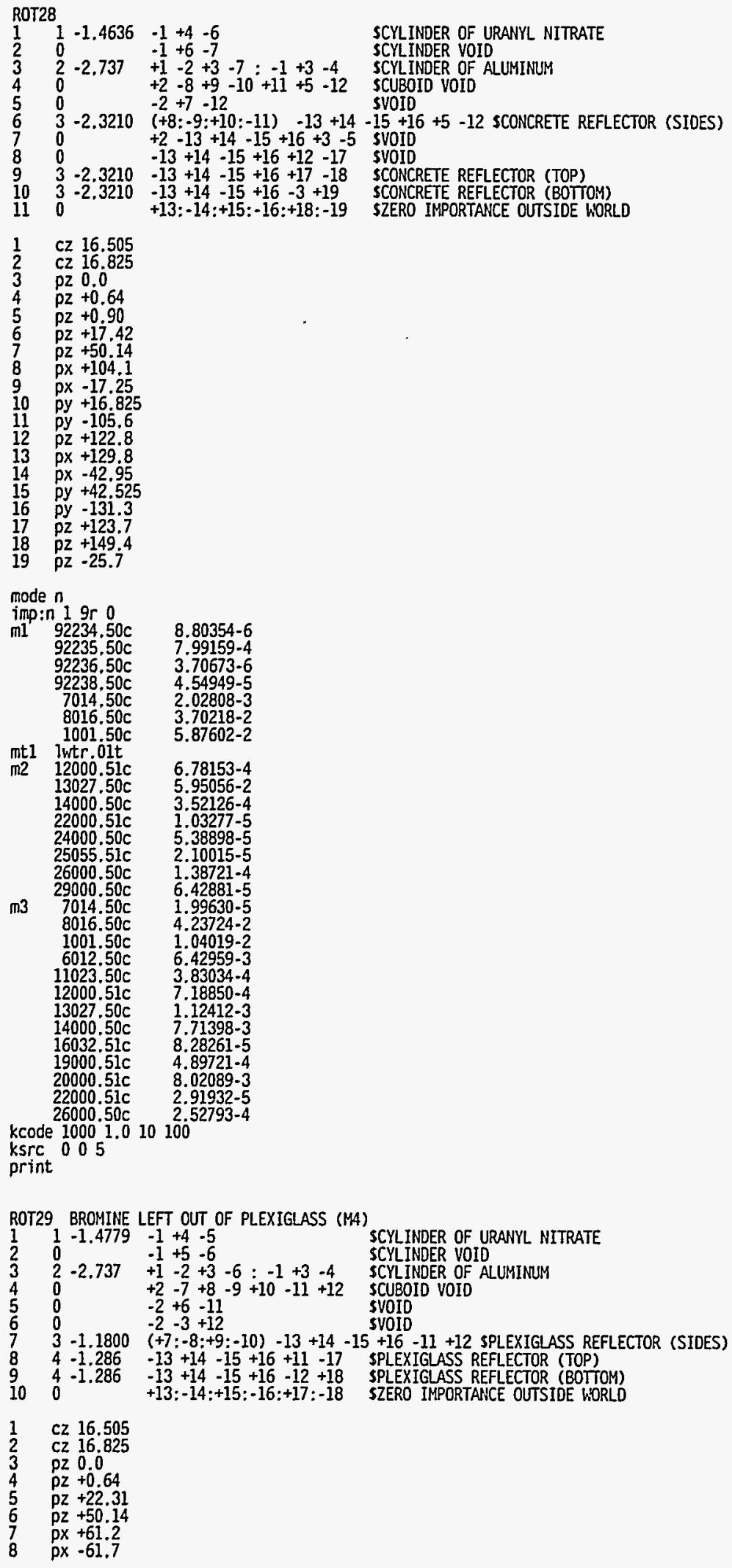



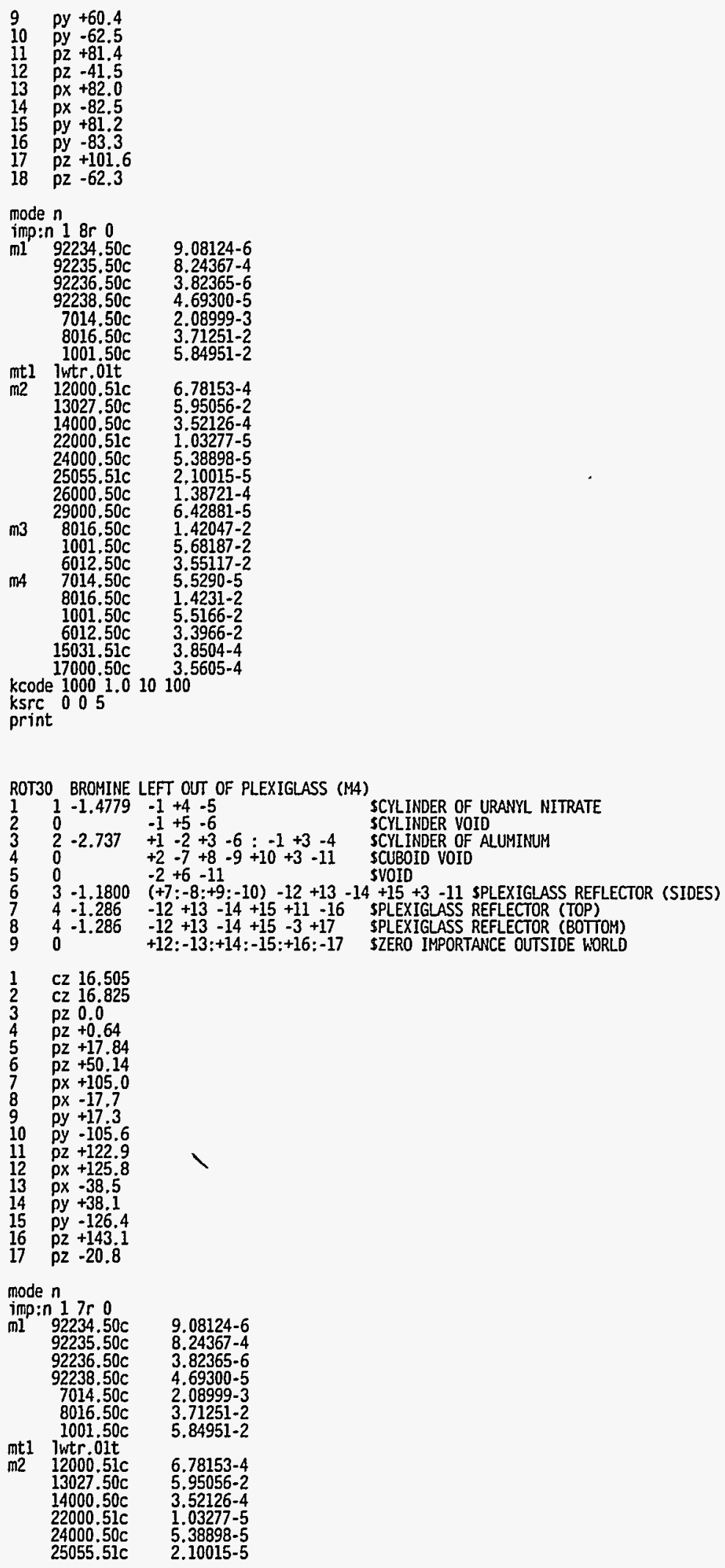

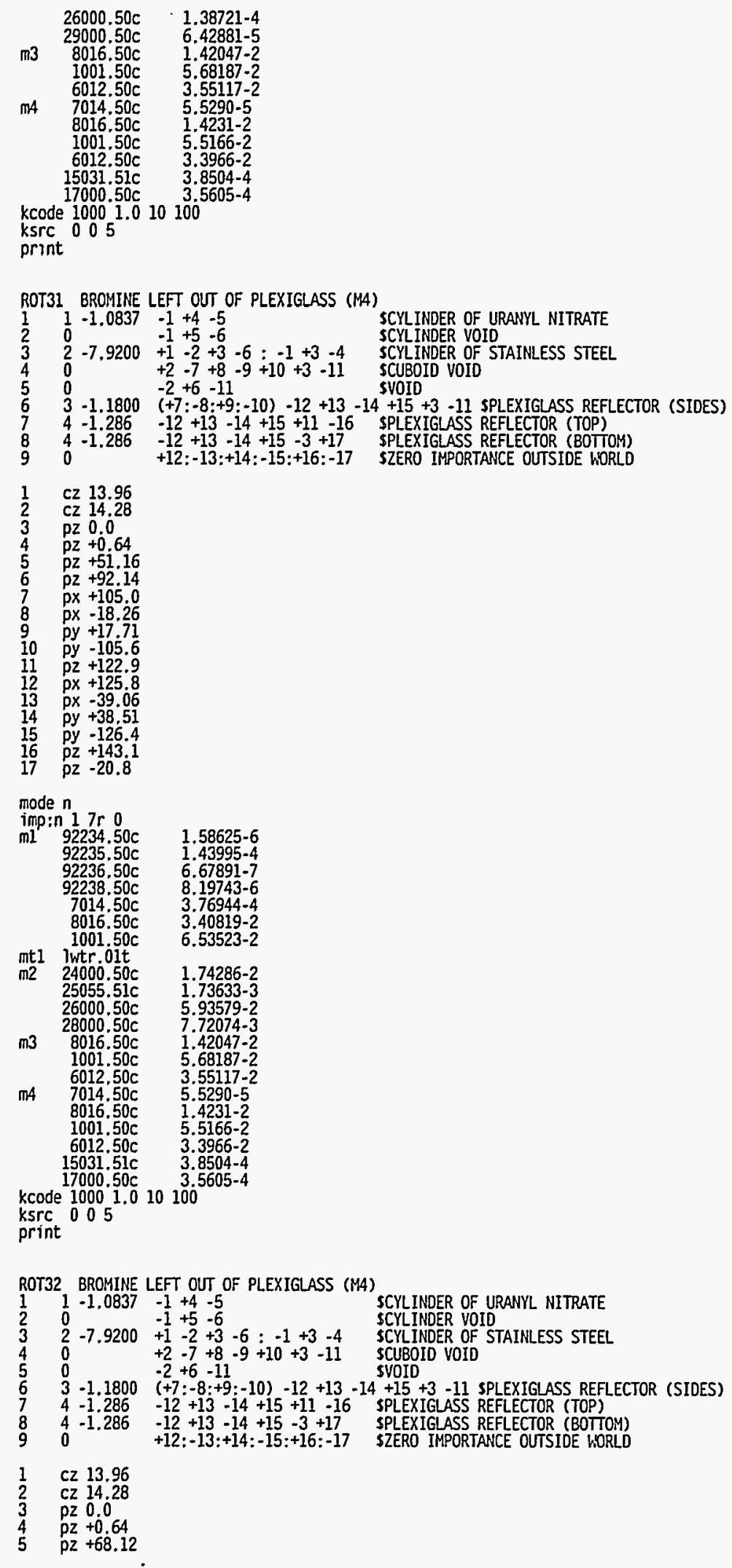

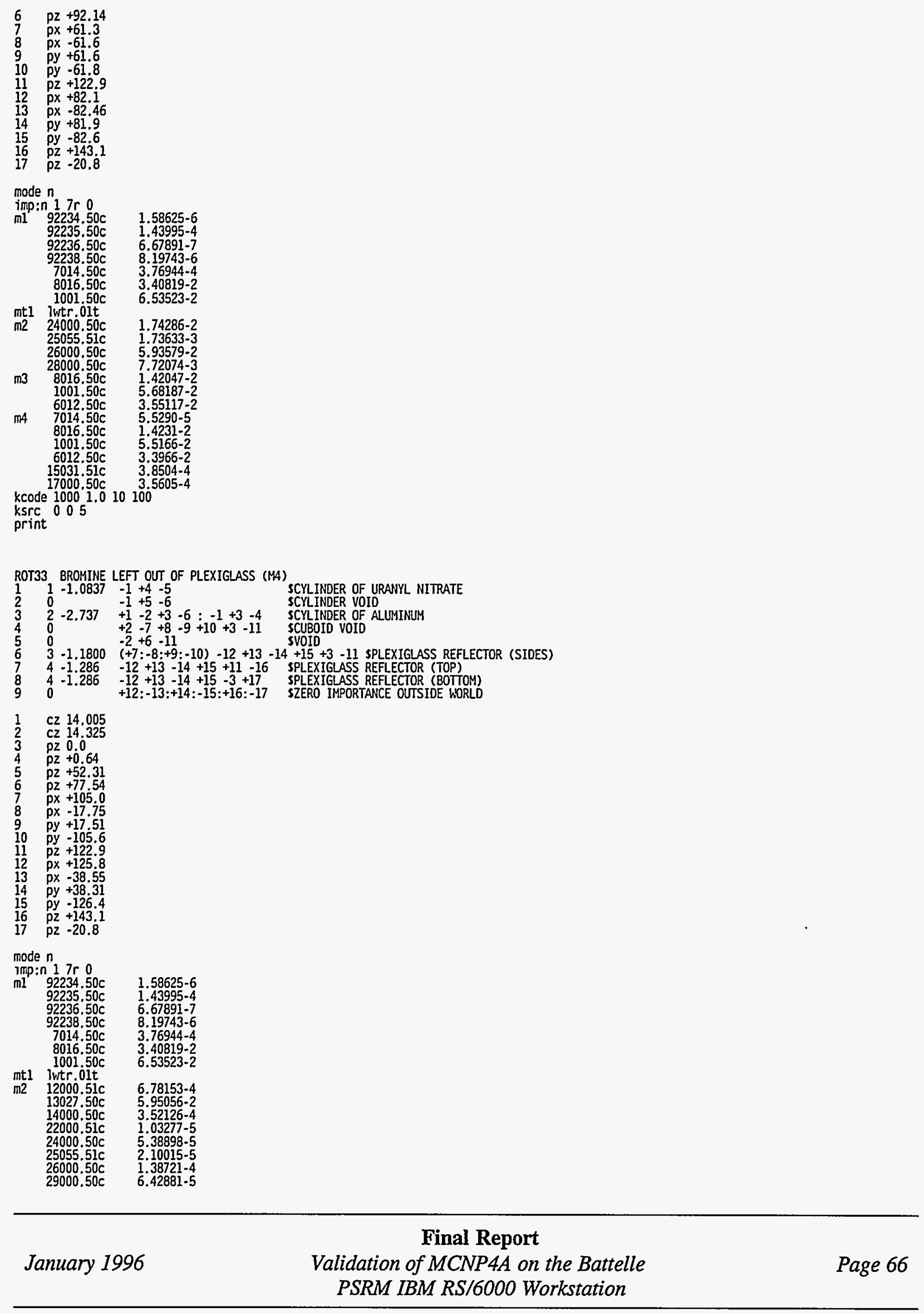
$\mathrm{m} 3 \quad 8016.50 \mathrm{c} \quad 1.42047-2$

$1001.50 \mathrm{C} \quad 5.68187-2$

$6012,50 \mathrm{C} \quad 3.55117-2$

$\mathrm{m4}$

$7014.50 \mathrm{C} \quad 5.5290-5$

$1001.50 \mathrm{C} \quad 5.5166-2$

$601250 \mathrm{c} \quad 3.5965-2$

$15031.51 \mathrm{c} \quad 3.8504-4$

17000.50

kcode 10001.010100

ksrc 005

print 


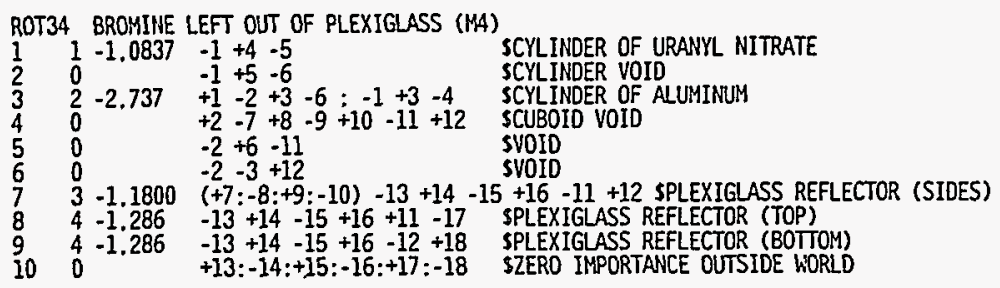

-13:-14:+15:-16:+17:-18 SZERO IMPORTANCE OUTSIDE LORLD

MITRATE

$\begin{array}{ll}1 & c z 16.505 \\ 2 & c z 16.825 \\ 3 & p z 0.0 \\ 4 & p z+0.64 \\ 5 & p z+34.97 \\ 6 & p z+50.14 \\ 7 & p x+61.2 \\ 8 & p x-61.7 \\ 9 & p y+60.4 \\ 10 & p y-62.5 \\ 11 & p z+81.4 \\ 12 & p z-41.5 \\ 13 & p x+82.0 \\ 14 & p x-82.5 \\ 15 & p y+81.2 \\ 16 & p y-83.3 \\ 17 & p z+101.6 \\ 18 & p z-62.3\end{array}$

mode $n$

imp:n 1 8r 0

$\mathrm{ml} 92234.50 \mathrm{c}$

$92235.50 \mathrm{c}$

$92236.50 \mathrm{C}$

$7014.50 \mathrm{c}$

$7014.50 c$

$1001.50 \mathrm{c}$

mt l iwtr.01t

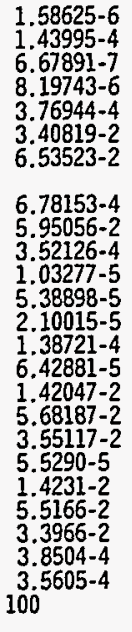

$26000.50 \mathrm{c}$

$3027.50 \mathrm{c}$

$14000.50 \mathrm{c}$

$22000.51 \mathrm{c}$

24000.50

25055.510

$1001.50 \mathrm{c}$

$6012.50 \mathrm{c}$

$7014.50 \mathrm{c}$

m4 7014.50c

$8016.50 \mathrm{c}$

$1001.50 \mathrm{C}$

$6012.50 \mathrm{c}$

$17000.50 \mathrm{c}$

kcode 10001.010100

print

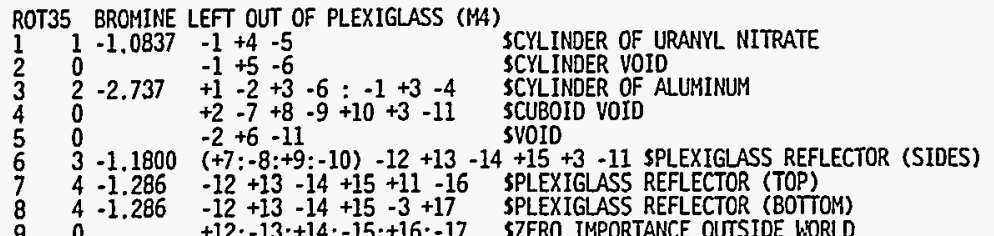

$\begin{array}{lll}4-1.286 & -12+13-14+15-3+17 & \text { SPLEXIGLASS REFLECTOR (BOTTOM) } \\ 0 & +12:-13:+14:-15:+16:-17 & \text { SZERO IMPORTANCE OUTSIDE WORLD }\end{array}$

$\frac{1}{2} \quad c z 16.505$

$2 \quad \mathrm{CZ} 16.825$

pz 0.04

$p z+0.64$

$\rho 2+28.34$

$p x+105.0$

$p x-17.72$

9 py +17.10

10 py -105.6

I1 $p Z+122.9$

px

14 py $+37,90$

January 1996

Final Report

Validation of MCNP4A on the Battelle

Page 68 PSRM IBM RS/6000 Workstation 
$15 \mathrm{py}-126.4$

$17 \mathrm{pz}-20 . \dot{8}$

mode $n$

imp:n $17 r \quad 0$

1.58625-6

(

$7014.50 \mathrm{C}$

$8016,50 \mathrm{C}$ $1001.50 \mathrm{c}$

mt1 lwtr.01t

$3.40819-2$
$6.53523-2$

6.67891-7

8.19743-6

$13027.50 \mathrm{c}$

6.78153-4

5. $95056-2$

3.52126-4

$14000.50 \mathrm{C} \quad 3.52126-4$

$22000.51 \mathrm{c}$

$24000.50 c$

$26000.50 \mathrm{C}$

$29000.50 \mathrm{c}$

2.10015-5

$1.38721-4$

$8016.50 \mathrm{c}$

$1001.50 \mathrm{c}$

$7014.50 \mathrm{c}$

$714.50 \mathrm{c}$

$1001.50 \mathrm{c}$

$1001.50 \mathrm{c}$

$15031.51 \mathrm{C}$

1. $42881-5$

$5.68187-2$

$3.55117-2$

5.5290 .5

$5.5290-5$
$1.4231-2$

$5.5166-2$

5.5166-2

$3.3966-2$

$3.8504-4$
$3.5605-4$

kcode 10001.010100

ksrc 005

print

ROT36 BROMINE LEFT OUT OF PLEXIGLASS (MA)

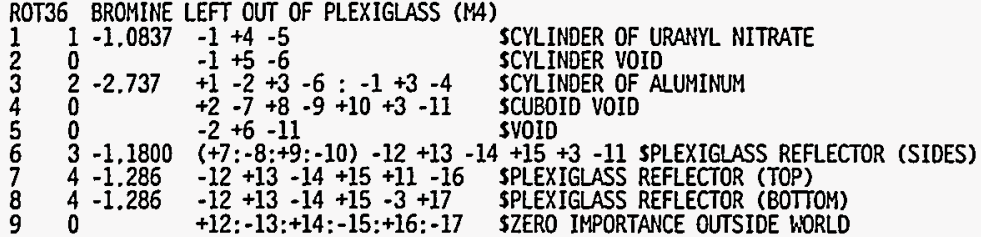

$\begin{array}{ll}1 & c z 16.505 \\ 2 & c z 16.825 \\ 3 & p z 0.0 \\ 4 & p z+0.64 \\ 5 & p z+32.39 \\ 6 & p z+50.14 \\ 7 & p x+61.3 \\ 8 & p x-61.6 \\ 9 & p y+61.1 \\ 10 & p y-61.8 \\ 11 & p z+122.9 \\ 12 & p x+82.1 \\ 13 & p x-82.4 \\ 14 & p y+81.9 \\ 15 & p y-82.6 \\ 16 & p z+143.1 \\ 17 & p z-20.8\end{array}$

mode $n$

imp: 1 1 $7 r \quad 0$

$92235.50 \mathrm{c}$

$92236.50 \mathrm{c}$

$7014.50 \mathrm{c}$

$8016.50 \mathrm{c}$

mtl iwtr.01t

$1.58625-6$

. $43995-4$

$6.67891-7$

$8.19743-6$

$3.40819-2$

$6.53523-2$

12000

$14000.50 \mathrm{C}$

$22000.51 \mathrm{c}$

$24000.50 \mathrm{C}$

$25055.51 \mathrm{C}$

$26000.50 \mathrm{C}$

$8016.50 \mathrm{c}$

$1001.50 \mathrm{c}$

政

6.78153-4

$6.78153-4$
$5.95056-2$

3.52126-4

$1.03277-5$

5.38898-5

2.10015 .5

$1.38721-4$

$6.42881-5$

.68187-2

$55117-2$

5. $5290-5$

$\begin{array}{ll}8016.50 \mathrm{c} & 1.4231-2 \\ 1001.50 \mathrm{c} & 5.5166-2\end{array}$

January 1996 
POEF-SH-33

$6012.50 \mathrm{C} \quad 3.3966-2$

$15031.51 \mathrm{C} \quad 3.8504-4$

$17000.50 \mathrm{c} \quad 3.5605-4$

kcode $10001.010100^{\circ}$

ksrc 005

print 


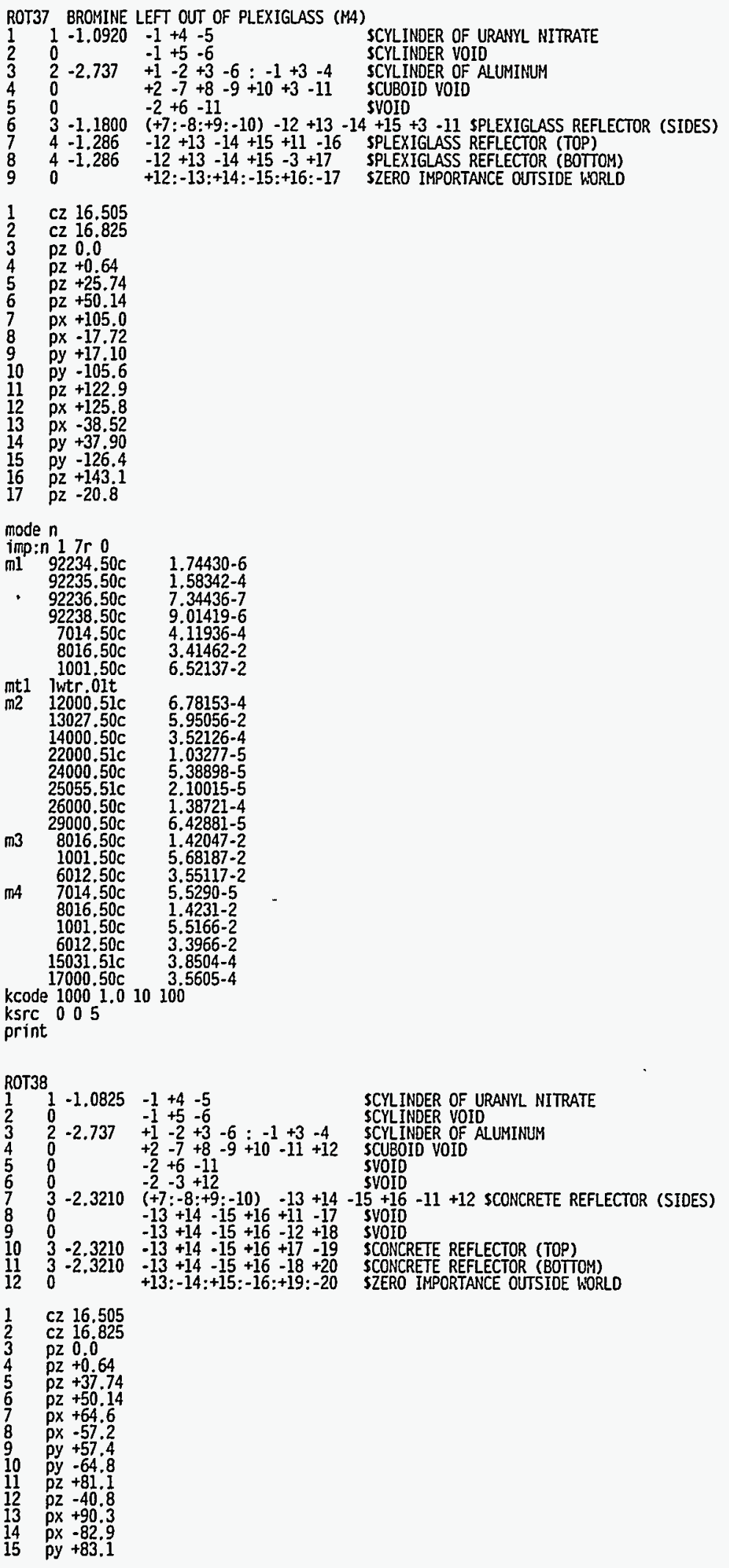

$\begin{array}{cc}\text { Final Report } \\ \text { January } 1996 & \begin{array}{c}\text { Validation of MCNP4A on the Battelle } \\ \text { PSRM IBM RS/6000 Workstation }\end{array} \\ \end{array}$



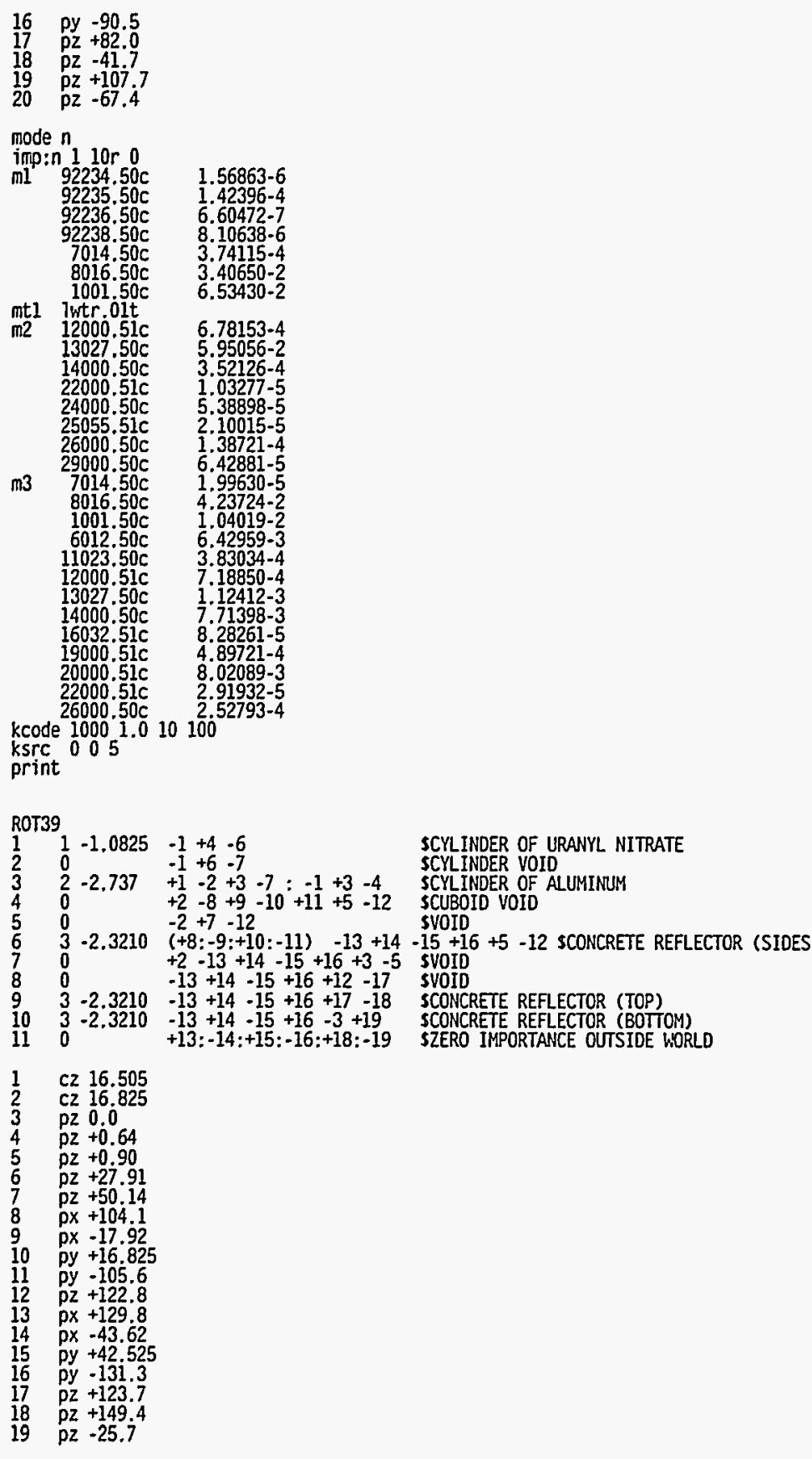

mode $n$,

\begin{tabular}{|c|c|c|}
\hline $\mathrm{ml}$ & $\begin{array}{r}19 r .0 \\
92234.50 \mathrm{c} \\
92235.50 \mathrm{c} \\
92236.50 \mathrm{c} \\
92238.50 \mathrm{c} \\
7014.50 \mathrm{c} \\
8016.50 \mathrm{c} \\
1001.50 \mathrm{c}\end{array}$ & $\begin{array}{l}1.56863-6 \\
1.42396-4 \\
6.60472-7 \\
8.10638-6 \\
3.74115-4 \\
3.40650-2 \\
6.53430-2\end{array}$ \\
\hline $\begin{array}{c}m t \\
m 2\end{array}$ & $\begin{array}{l}\text { lwtr.01t } \\
12000.51 \mathrm{c} \\
13027.50 \mathrm{c} \\
14000.50 \mathrm{c} \\
22000.51 \mathrm{c} \\
24000.50 \mathrm{c}\end{array}$ & $\begin{array}{l}6.78153-4 \\
5.95056-2 \\
3.52126-4 \\
1.03277-5 \\
5.38898-5\end{array}$ \\
\hline
\end{tabular}



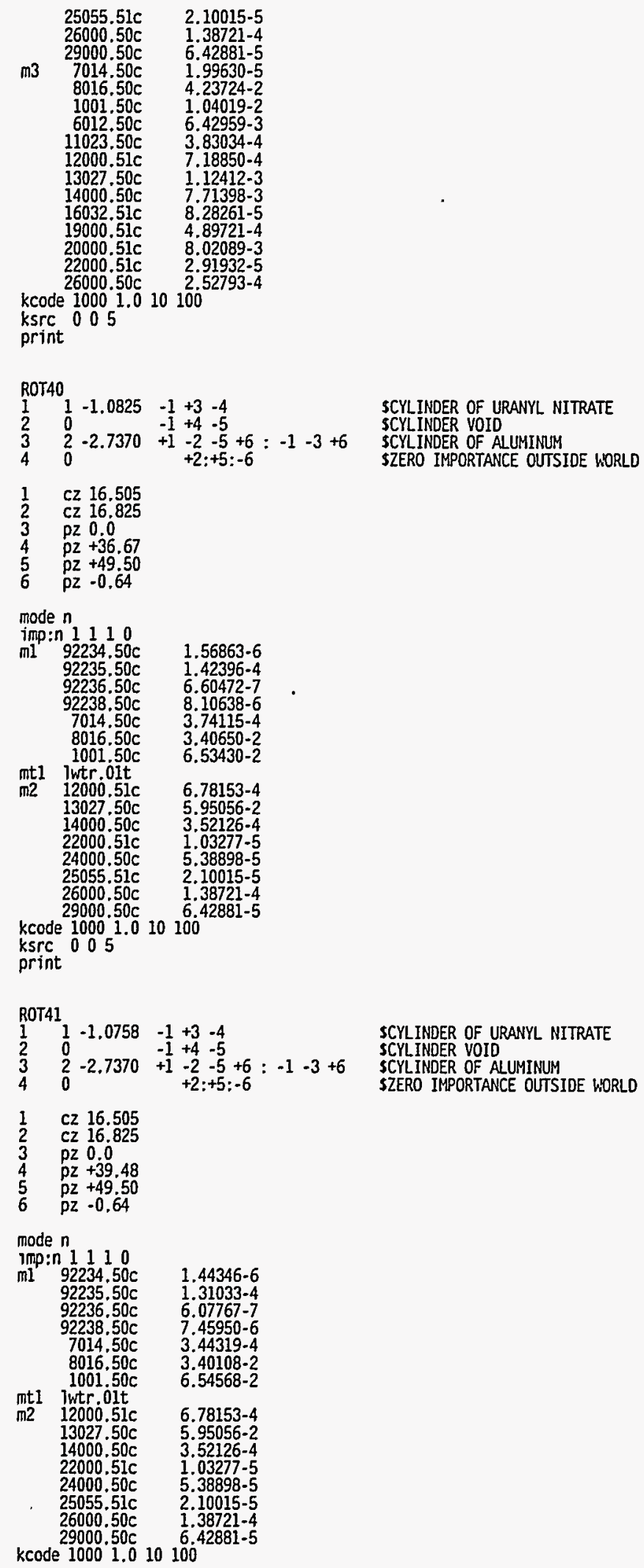

Final Report

January 1996 
ksrc 005

print

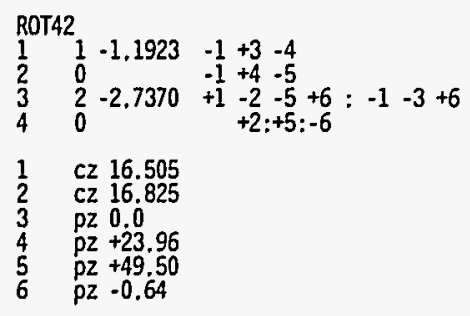

mode $n$

imp:n 1110

$\mathrm{ml}$ : $92234,50 \mathrm{c}$

$92235.50 \mathrm{c}$

$92236.50 \mathrm{c}$

$2238.50 c$

$7014.50 \mathrm{c}$

$8016.50 \mathrm{c}$

3.61325-6

3. $27999-4$

1.52136-6

$8.76449-4$

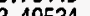

6. $34137-2$

mtI

iwtr.01t

$12000.51 \mathrm{c}$

6.78153-4

5.95056-2

3.52126-4

$1.03277-5$

5.38898-5

$2.10015-5$

$1.38721-4$

24000.5

$2555.51 \mathrm{c}$

2000 $50 \mathrm{c}$

6. $42881-5$

kcode 10001010100

ksrc 005

ksrc
print

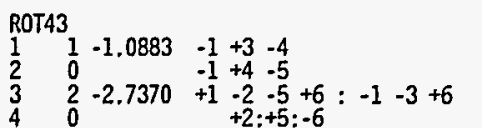

SCYLINDER OF URANYL NITRATE

SCYLINDER VOID

CYLINDER OF ALUMINUM

SZERO IMPORTANCE OUTSIDE WORLD

\section{$1 \quad C 225.345$ \\ CZ 25.665 \\ pz 0.0 \\ pz +20.48 \\ $\begin{array}{ll}p z & +30.9 \\ p z & -0.64\end{array}$}

mode $n$

imp:n 1110

$\mathrm{ml} 92234.50 \mathrm{C} \quad 1.68171-6$

$92235.50 \mathrm{C} \quad 1.68171-6$

92236.50c $\quad 7.08084 .7$

$92238.50 \mathrm{c} \quad 8.69075 .6$

$7014.50 \mathrm{c} \quad 3.94328-4$

$1001.50 \mathrm{C} \quad 6.52634 .2$

mtl lwtr.01t

$\begin{array}{lll}\mathrm{m} 2 & 12000.51 \mathrm{c} & 6.78153-4 \\ & 13027.50 \mathrm{c} & 5.95056-2\end{array}$

$14000.50 \mathrm{c}$

$22000.51 \mathrm{C} \quad 1.03277-5$

$24000.50 \mathrm{C} \quad 5.38898-5$

25055.51C 2.10015-5

$26000.50 \mathrm{c} \quad 1.38721-4$

$50 \mathrm{c} \quad 6.42881-5$

kcode 100011.010100

ksre 005

print

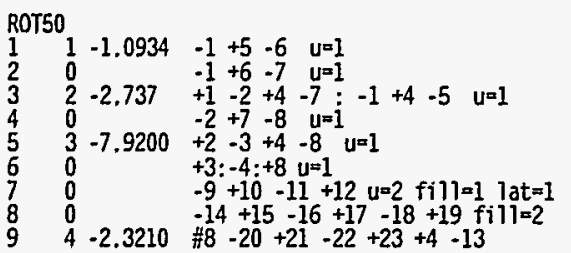

SCYLINDER OF URANYL NITRATE

SCYLINDER VOID

SCYLINDER OF ALUMINUM

SVOID

SVOID

SCELL FILLED WITH 1-6

\$4X XX1 ARRAY

SCONCRETE REFLECTOR (SIDES)

January 1996

Final Report

Validation of MCNP4A on the Battelle

Page 74 PSRM IBM RS/6000 Workstation 

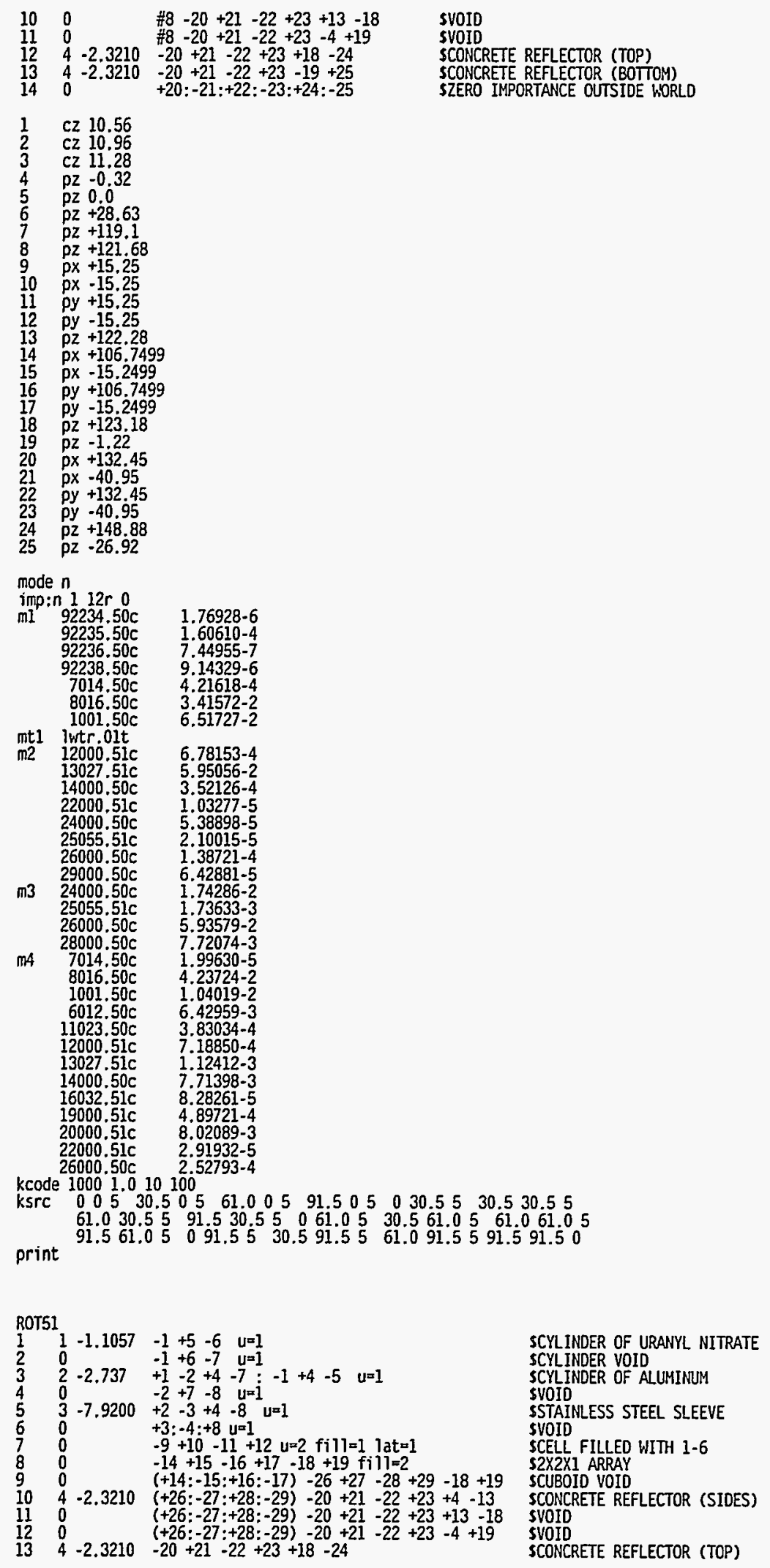

January 1996

Final Report 

$\begin{array}{lll}14 & 4 & -2.3210 \\ 15 & 0 & \end{array}$
Cz 10.56
CZ 10.96
C2 11,28
pz -0.32
pz 0.0
pz $+60,7$
pz +119.1
$\mathrm{px}+15.25$
px $-15,25$
py +15.25
py $-15,25$
$p z+122.28$
$\mathrm{pX}+45.7499$
$\mathrm{px}-15.2499$
py +45.7499
py -15.2499
$p z+123.18$
$\mathrm{pz}-1.22$
$\mathrm{px}+101.95$
px -71.45
py +101.95
py -71.45
pz + 148.88
$p z-26.92$
$\mathrm{px}+76.25$
$\mathrm{px}-45.75$
py +76.25
py -45.75

$-20+21-22+23-19+25$

mode $n$

imp: $113 r$

$m i$

\section{$92234.50 \mathrm{C} \quad 2.00096-6$}

$92235.50 \mathrm{c}$

$92236.50 \mathrm{c}$

2238.50

7014.50

8016.50

$1001.50 \mathrm{c}$

mtl lwtr.01t

$12000.51 \mathrm{c}$

$13027.50 \mathrm{C}$

$14000.50 \mathrm{c}$

$22000.51 \mathrm{C}$

$24000.50 \mathrm{c}$

$25055.51 \mathrm{c}$

$26000.50 \mathrm{C}$

$29000.50 \mathrm{C}$

24000.50

$25055.51 c$

$26000.50 \mathrm{c}$

28000.50

$m 4$

$7014.50 \mathrm{C}$

8016.50

001.50

$6012.50 \mathrm{c}$

11023.50

$12000.51 \mathrm{c}$

$1302750 \mathrm{c}$

$14000.50 \mathrm{c}$

1603251

9000.51

1900.51

2000.51

$22000.51 \mathrm{c}$

kcode 1000 1. 0010100

$1.81641-4$

$8.42503-7$

$1.03406-5$

4.72154-4

$3.42563-2$

6.49829 .2

6.78153-4

$5.95056-2$

$3.52126-4$

$1.03277-5$

5. 38898-5

1. $38721-4$

$1.38721-4$

$6.42881-5$

$1.74286-2$

$5.93579-2$

7.72074-3

$1.99630-5$

$4.23724-2$

$1.04019-2$

6.42959-3

$3.83034-4$

$7.18850-4$

$1.71398-3$

$8.28261-5$

$89721-4$

$02009-3$

$2.91932-5$

2.52793.4

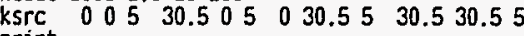

print

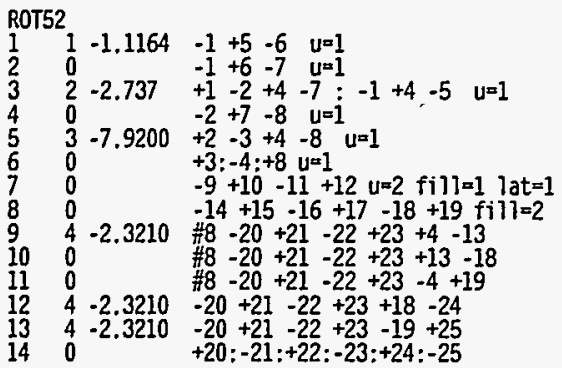

SCYLINDER OF URANYL NITRATE

SCYLINDER VOID

SCYLINDER OF ALUMIMUM

SVOID

SSTAINLESS STEEL SLEEVE

SVOIO

SCELL FILLED WITH 1-6

$54 \times 4 \times 1$ ARRAY

SCONCRETE REFLECTOR (SIDES)

SVOID

SVOID

SCONCRETE REFLECTOR (TOP)

SCONCRETE REFLECTOR (BOTOY)

SZERO IMPORTANCE OITSIDE WORLD
SCONCRETE REFLECTOR (BOTTOM)

SZERO IMPORTANCE OUTSIDE WORLD 

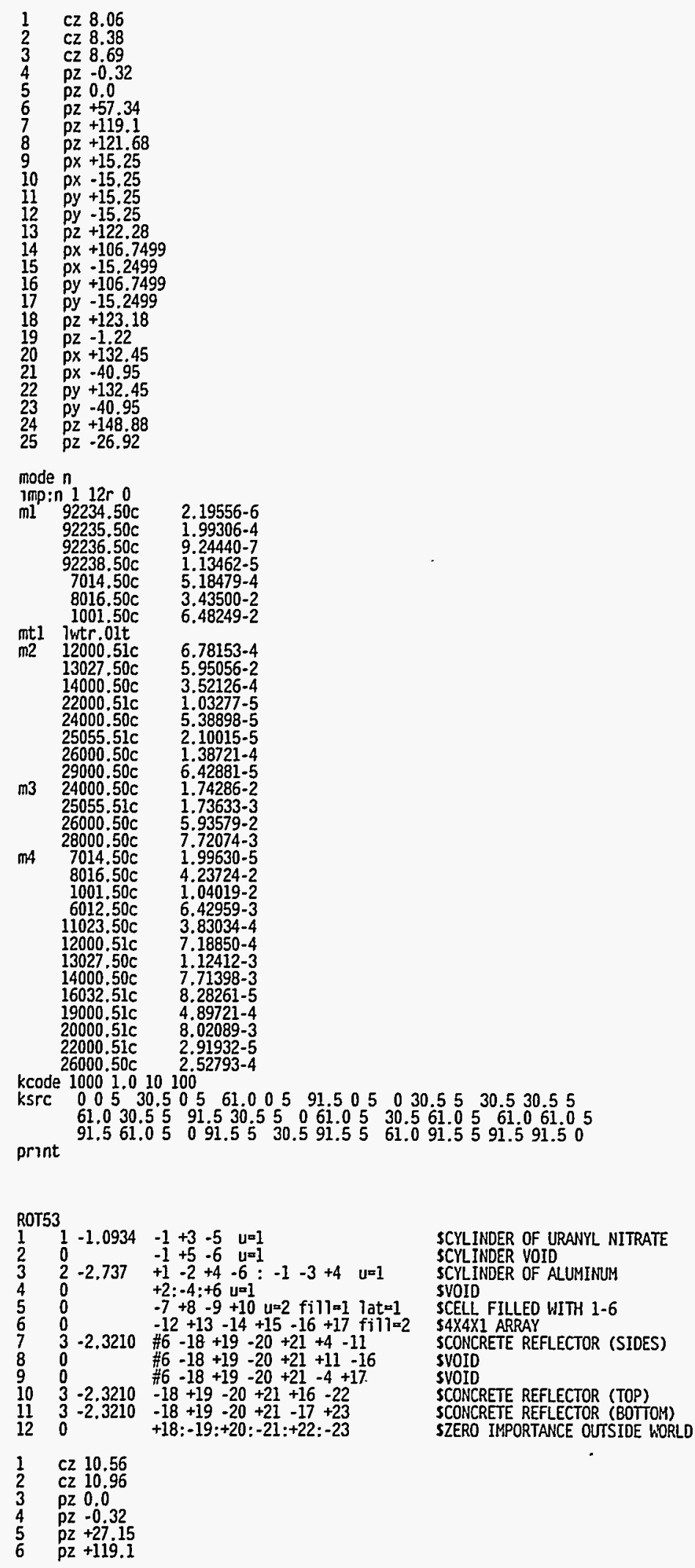

Final Report 

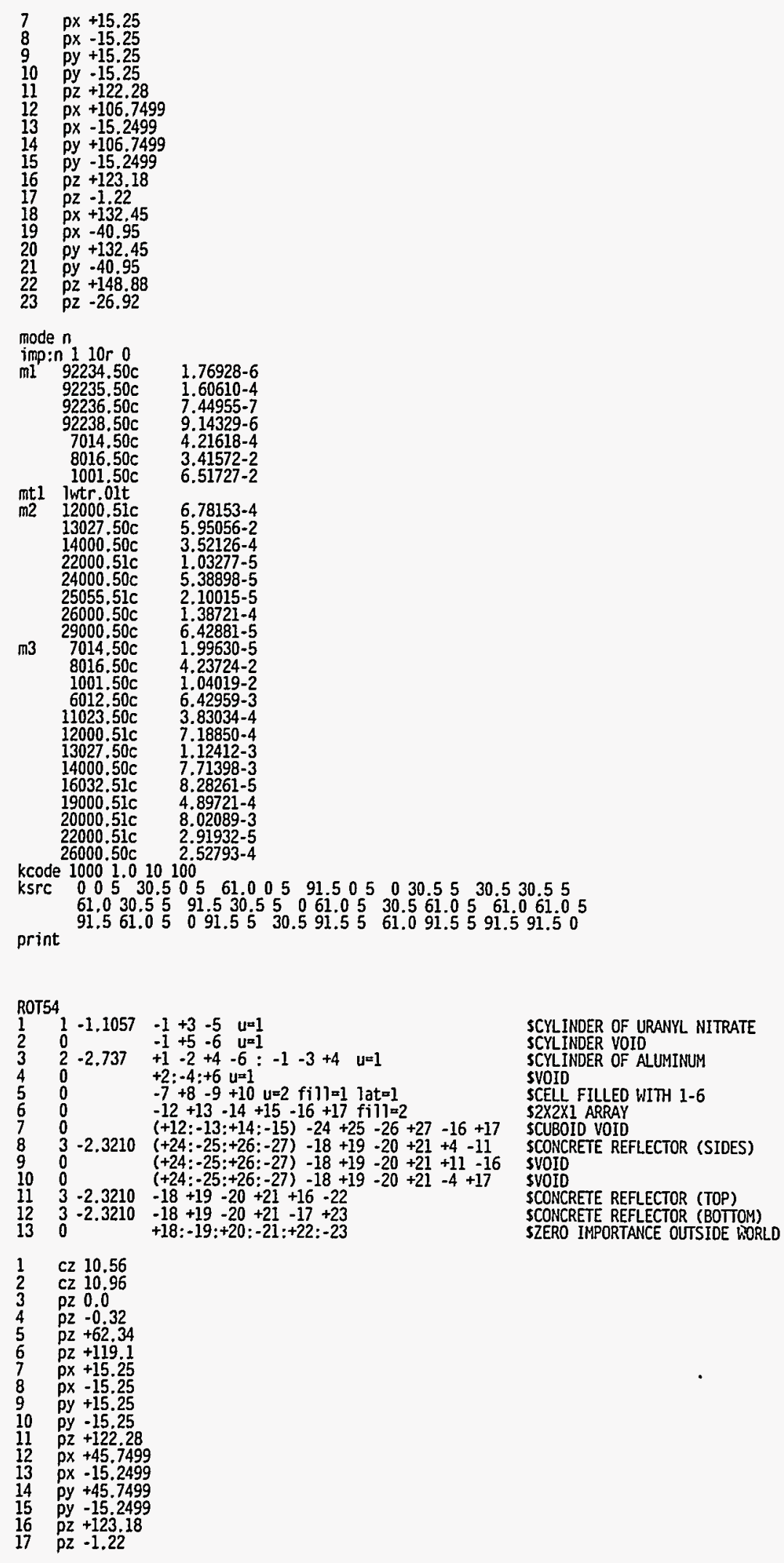


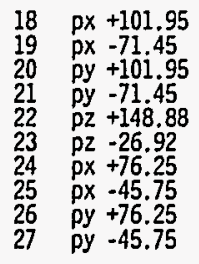

mode $n$

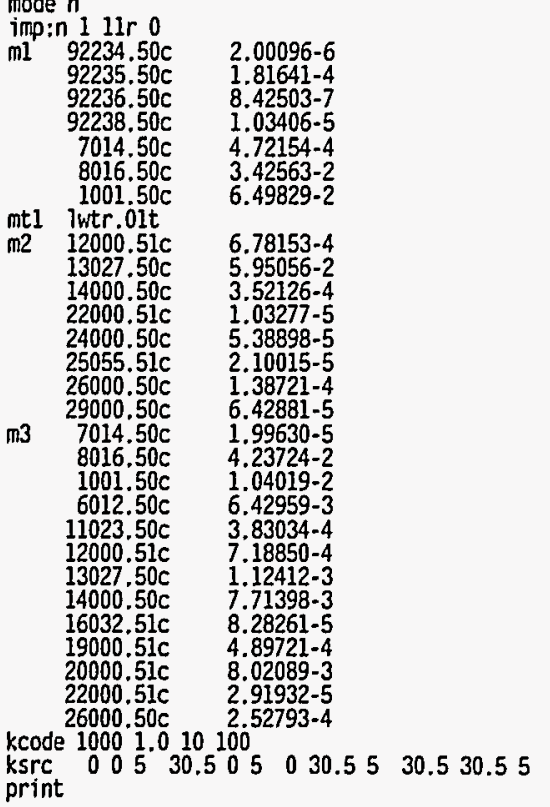

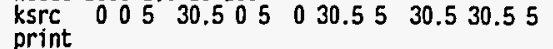

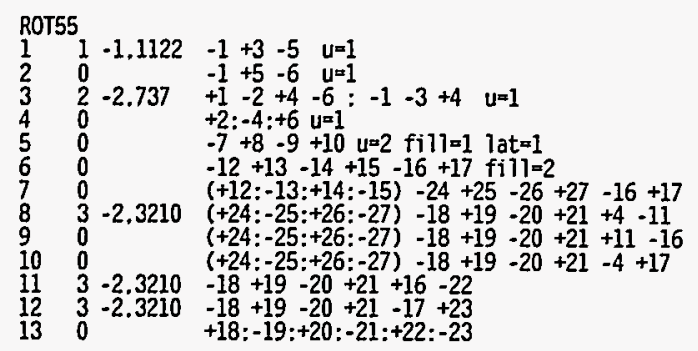

SCYLINDER OF URANYL NITRATE SCYLINDER VOID
SCYLINDER OF ALUMINUM SVOID CELL FILLED WITH 1-6 S2X2X1 ARRAY SCUBOIO VOID SCONCRETE REFLECTOR (SIDES) SVOID SCONCRETE REFLECTOR (TOP) CONCRETE REFLECTOR (BOTTOM) SZERO IMPORTANCE OUTSIDE KORLD 
mode $n$

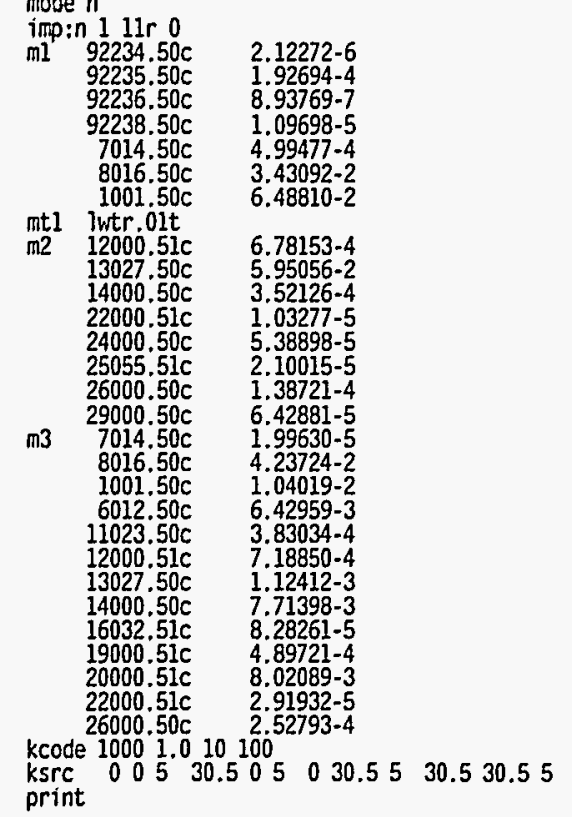

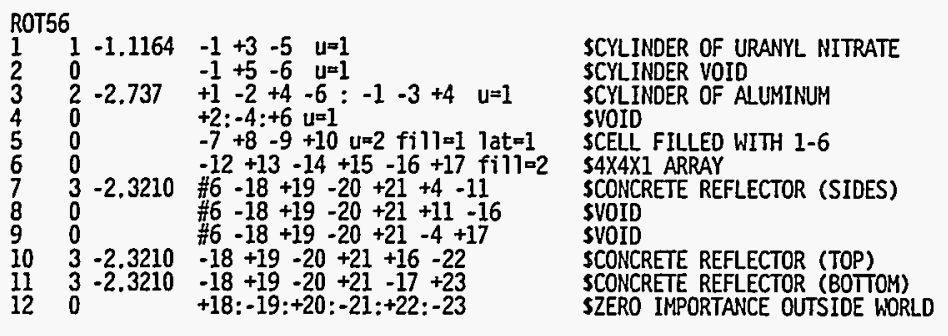

C2 8.06

$2 \quad$ CZ 8.38

3 pz 0.0

$5 \quad p z+5 i .21$

$6 \quad \mathrm{pz}+119.1$

7 px +15.25

8 px -15.25

9 py +15.25

pz +122.28

px +106.7499

px $-15,2499$

px -15.2499

py +106.7499

$p z+123,18$

pz -1.22

$p x+132.45$

px -40.95

py +132.45

py -40.95

pz +148.88

pz -26.92

mode $\mathrm{n}$
mimp: $1 \frac{1}{92234.50 \mathrm{c}} \mathrm{c}$
$92235.50 \mathrm{c}$
$92236.50 \mathrm{c}$
$92238.50 \mathrm{c}$
$7014.50 \mathrm{C}$
$8016.50 \mathrm{c}$
mtl lwtr.01t
$12000.51 \mathrm{c}$
$13027.50 \mathrm{C}$
$2000.51 \mathrm{C}$
$24000.50 \mathrm{c}$
25055.51c

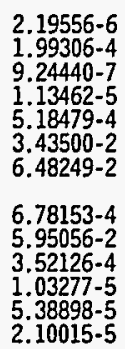



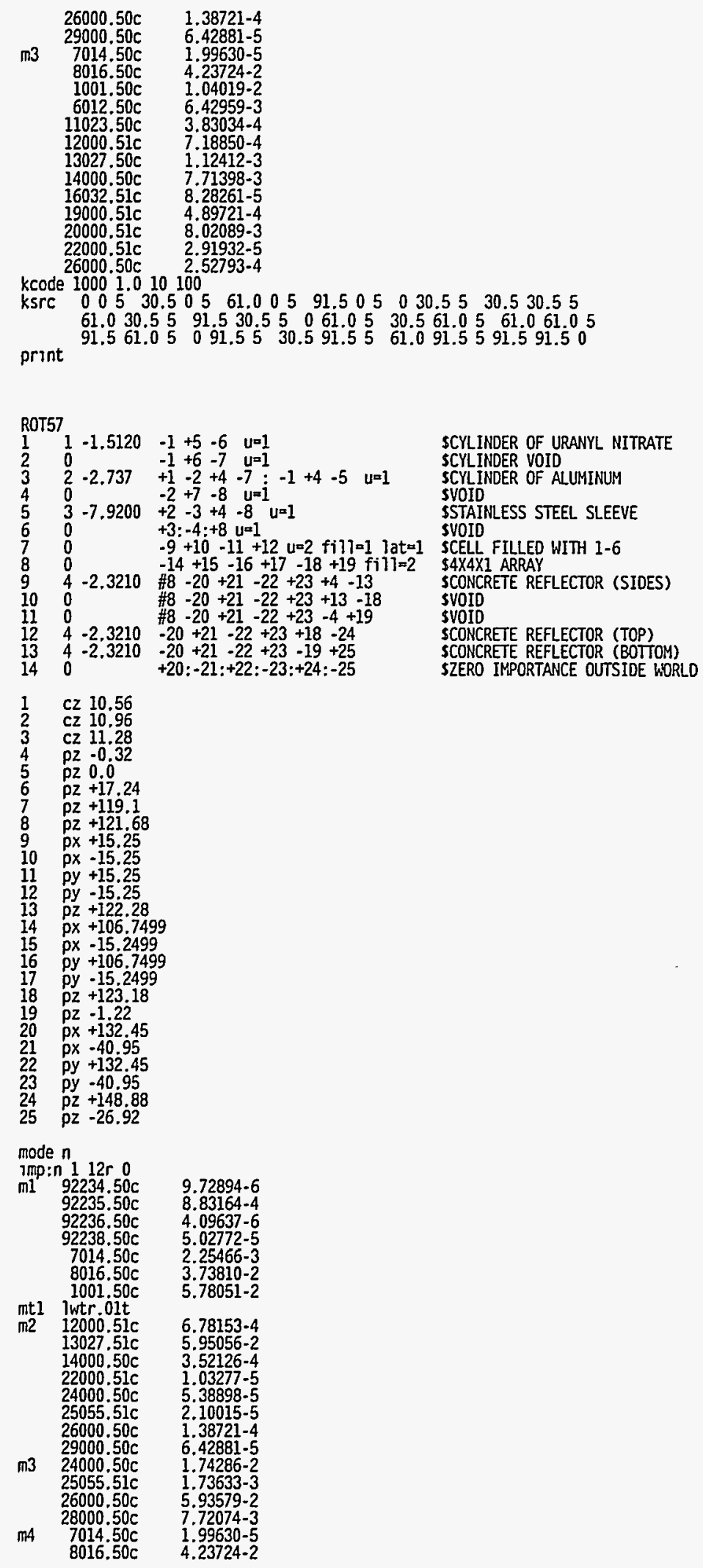
POEF-SH-33

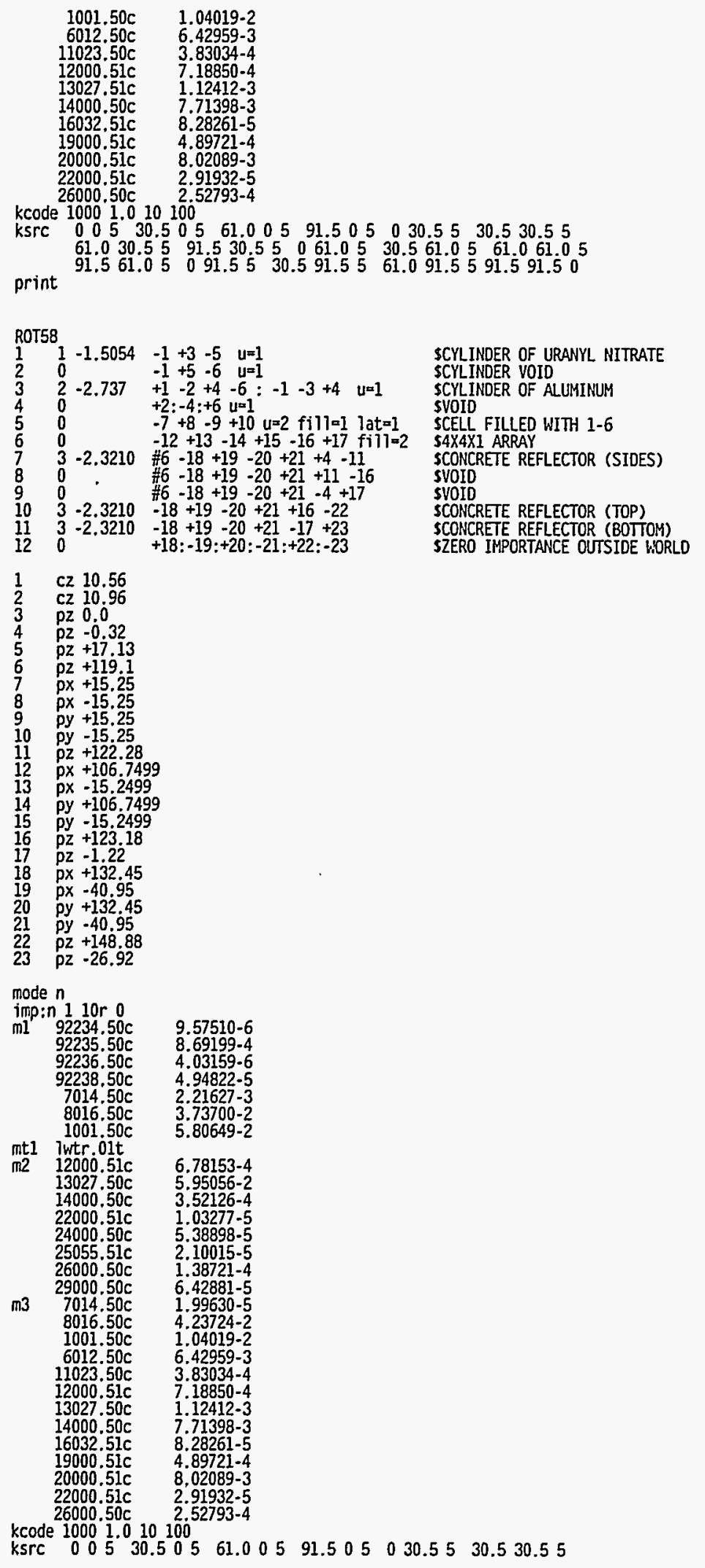

Final Report

January 1996 
$\begin{array}{lllllllll}61.0 & 30.55 & 91.5 & 30.55 & 0 & 61.05 & 30.5 & 61.05 & 61.061 .05\end{array}$

print

91.561 .05091 .5530 .591 .5561 .091 .5591 .591 .50

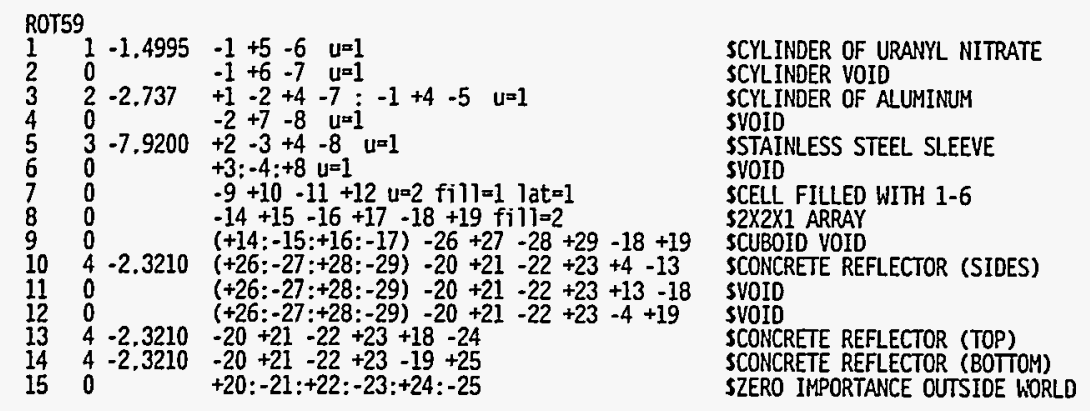

$\begin{array}{ll}1 & c z 10.56 \\ 2 & c z 10.96 \\ 3 & c z 11.28 \\ 4 & p z-0.32 \\ 5 & p z 0.0 \\ 6 & p z+29.49 \\ 7 & p z+119.1 \\ 8 & p z+121.68 \\ 9 & p x+15.25 \\ 10 & p x-15.25 \\ 11 & p y+15.25 \\ 12 & p y-15.25 \\ 13 & p z+122.28 \\ 14 & p x+45.7499 \\ 15 & p x-15.2499 \\ 16 & p y+45.7499 \\ 17 & p y-15.2499 \\ 18 & p z+123.18 \\ 19 & p z-1.22 \\ 20 & p x+101.95 \\ 21 & p x-71.45 \\ 22 & p y+101.95 \\ 23 & p y-71.45 \\ 24 & p z+148.88 \\ 25 & p z-26.92 \\ 26 & p x+76.25 \\ 27 & p x-45.75 \\ 28 & p y+75.25 \\ 29 & p y-45.75\end{array}$

mode $n$

1mp: $1113 r$ o

$\begin{array}{ll}92234.50 \mathrm{c} & 9.47675-6 \\ 92235.50 \mathrm{c} & 8.60271-4\end{array}$

$8.60271-4$

$92238.50 \mathrm{C}$

$7014.50 \mathrm{C}-2.19772-3$

$8016.50 \mathrm{C}$

$1001.50 \mathrm{C} \quad 5.80827-2$

mtl Iwtr. $01 \mathrm{t}$

$5.95056-2$

$3.52126-4$

5. $383898-5$

(2000

$22000.51 \mathrm{c}$

$24000.50 \mathrm{c}$

$25055.51 \mathrm{C}$

2.10015-5

$1000{ }^{50 \mathrm{C}} 1.38721-4$

$\mathrm{m} 3$

$25055.51 \mathrm{C}$

$2800050 \mathrm{c}$

$7014.50 \mathrm{c}$

$7014.50 \mathrm{c}$

$800.50 \mathrm{c}$

$1001.50 \mathrm{C}$

$6012.50 \mathrm{C}$

$11023.50 \mathrm{C}$

$12000.51 \mathrm{C}$

$13027.50 \mathrm{c}$

$14000.50 \mathrm{C}$

$16032.51 \mathrm{c}$

$19000.51 \mathrm{c}$

$2000051 \mathrm{c}$

$22000.51 \mathrm{c}$

$6.42881-5$

$1.74286-2$

$1.73633-3$

$5.93579-2$

$7.72074-3$

1. $99630-5$

1. $04019-2$

$6.42959-3$

3.83034-4

$7.18850-4$

1.12412 .3

$7.71398-3$

$4.89721-4$

.

. $91932-5$

52793-4

kcode 10001.010100 


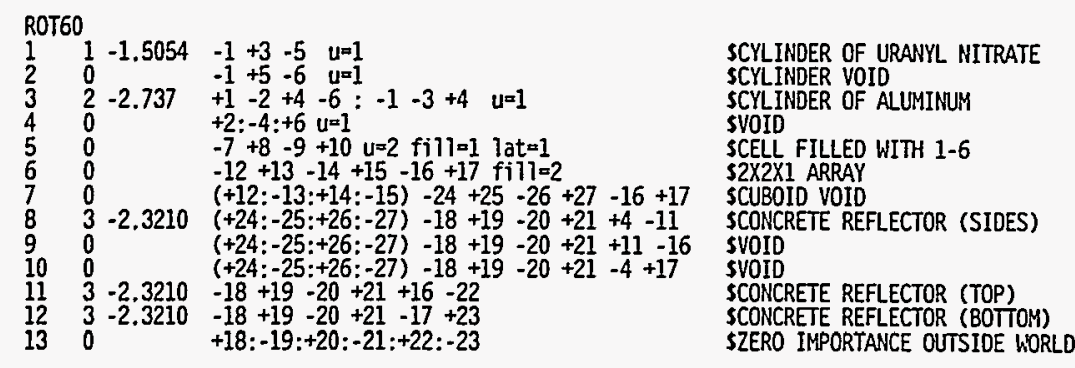

SCYLINDER VOID

DER OF ALUMINUM

SCELL FILLED WITH 1-6

\$2X2X1 ARRAY

(SIDES)

ECTOR (BOTTOH)

SZERO IMPORTANCE OUTSIDE WORLD

$$
\begin{array}{ll}
1 & c z 10.56 \\
2 & c z 10.96 \\
3 & p z 0.0 \\
4 & p z-0.32 \\
5 & p z+31.11 \\
6 & p z+119.1 \\
7 & p x+15.25 \\
8 & p x-15.25 \\
9 & p y+15.25 \\
10 & p y-15.25 \\
11 & p z+122.28 \\
12 & p x+45.499 \\
13 & p x-15.2499 \\
14 & p y+45.7499 \\
15 & p y-15.299 \\
16 & p z+123.18 \\
17 & p z-1.22 \\
18 & p x+101.95 \\
19 & p x-71.45 \\
20 & p y+101.95 \\
21 & p y-71.45 \\
22 & p z+148.88 \\
23 & p z-26.92 \\
24 & p x+76.25 \\
25 & p x-45.75 \\
26 & p y+76.25 \\
27 & p y-45.75
\end{array}
$$

\section{mode $n$}

imp:n $111 \mathrm{r} 0$

mi $=n 12234.50 \mathrm{c}$

\subsection{0 $\quad 9.57510-6$}

$92238.50 \mathrm{c} \quad 4.03159-6$

$7014.50 \mathrm{c} \quad 2.21627-3$

$8016.50 \mathrm{C} \quad 3.73700-2$

mt1 1001.50

lwtr.01t

$3.73700-2$
$5.80649-2$

$\mathrm{m2} 12000.51 \mathrm{c} \quad 6.78153-4$

$14000.50 \mathrm{C}$

$22000.51 \mathrm{C} \quad 1.03277-5$

$24000.50 \mathrm{C}$

$25055.51 \mathrm{c}$

$26000.50 \mathrm{c}$

$29000.50 \mathrm{c}$

$\mathrm{m} 3$

$7014.50 \mathrm{c}$

$8016.50 \mathrm{c}$

$1001.50 \mathrm{c}$

$6012.50 \mathrm{c}$

$11023.50 \mathrm{c}$

$12000.51 \mathrm{c}$

$13027.50 \mathrm{c}$

$14000.50 \mathrm{c}$

$16032.51 \mathrm{C}$

$20000.51 \mathrm{c}$

$22000.51 \mathrm{c}$

$26000.50 \mathrm{C}$

5.38898-5

2. $10015-5$

$1.38721-4$

6.42881-5

$1.99630-5$
$4.23724-2$

$1.04019-2$

$6.42959-3$

$3.83034-4$

$7.18850-4$

$1.12412-3$

7.71398-3

$8.28261-5$

$4.89721-4$

$8.02089-3$

$2.52793-4$

kcode 10001.010100

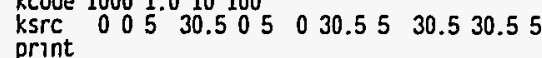

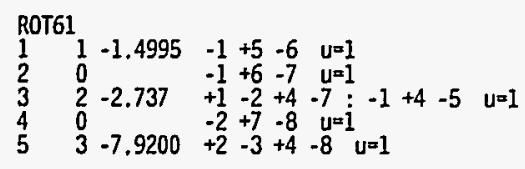

SCYLINDER OF URANYL NITRATE

SCYLINDER VOID

SCYLINDER OF ALUMINUM

SSTAINLESS STEEL SLEEVE 


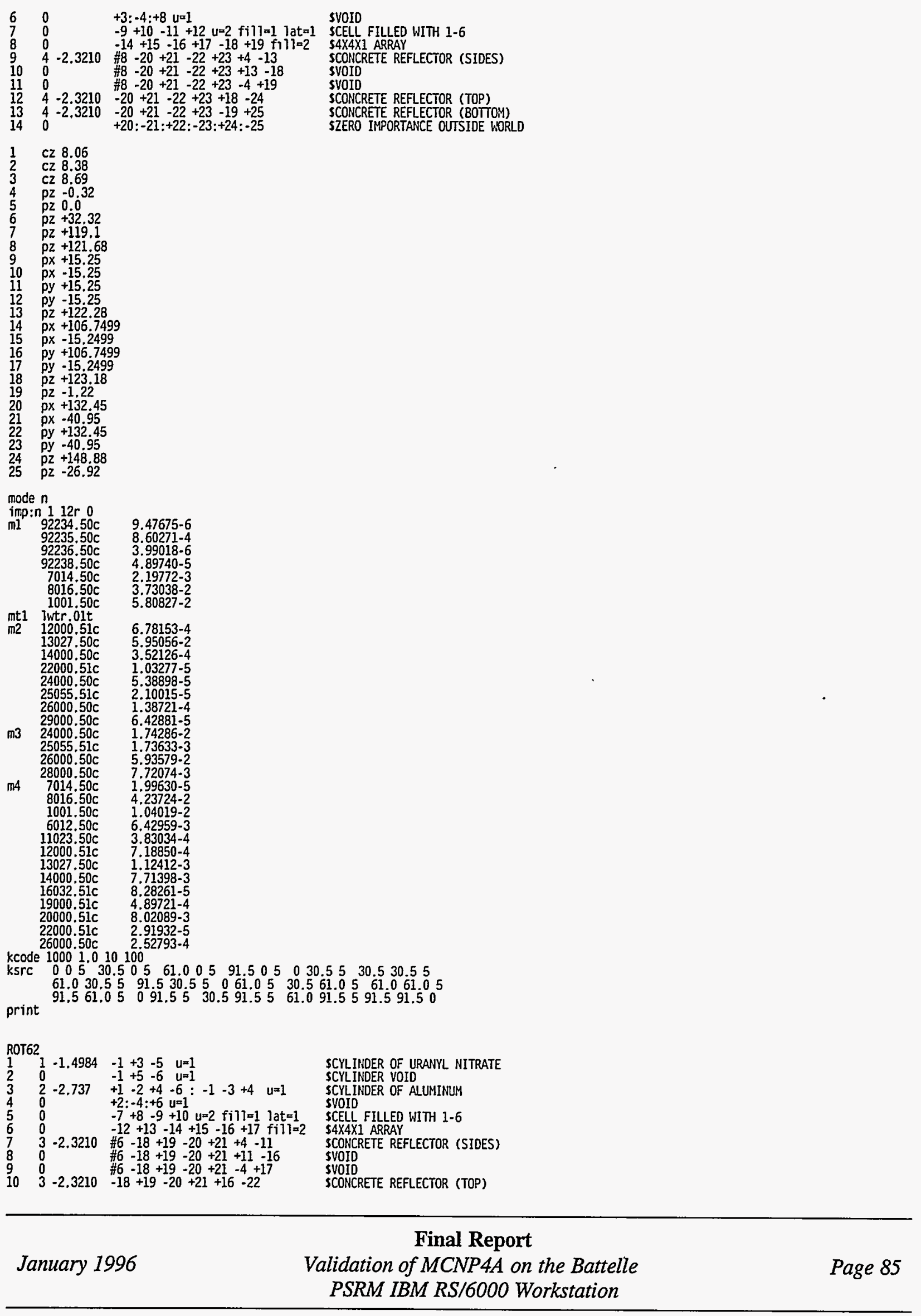



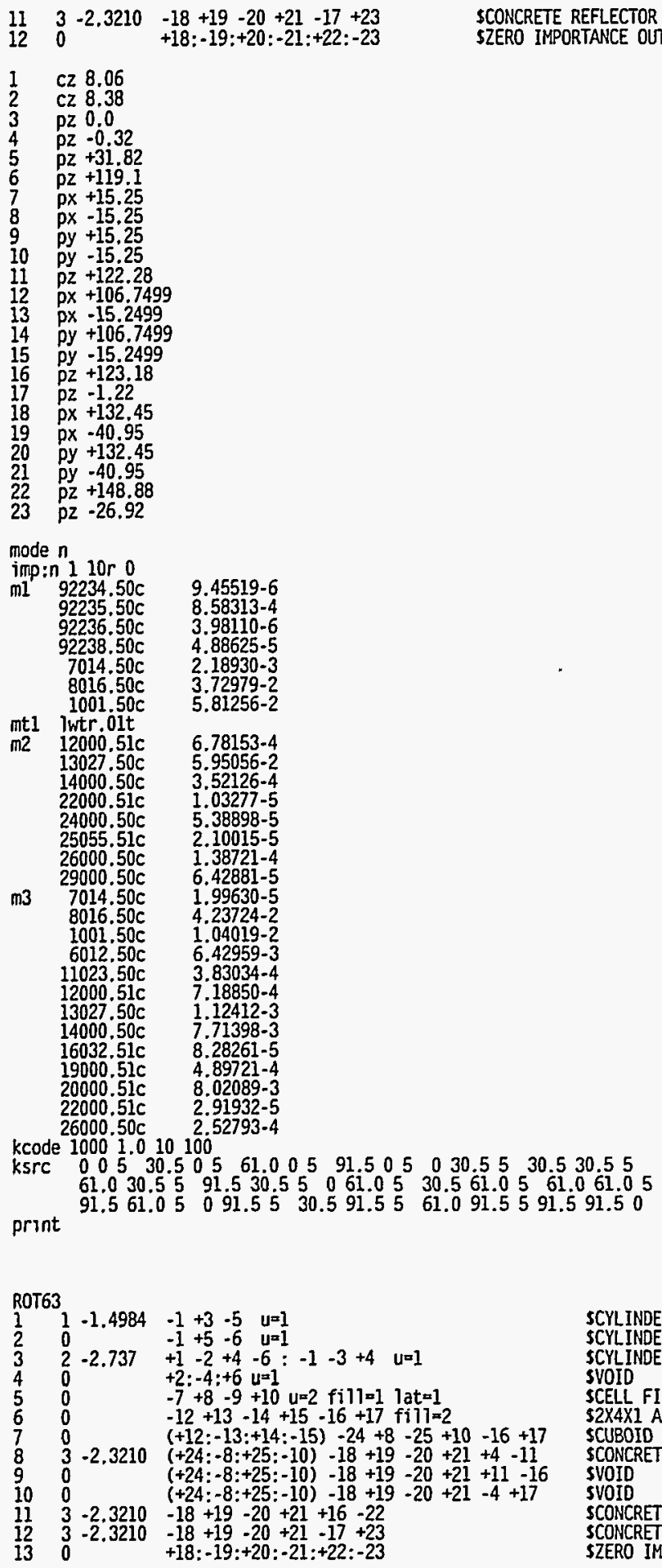

SCYLINDER OF URANYL NITRATE
SCYLINDER VOID
SCYLIIDER OF ALUMINUM
SVOID
SCELL FILLED WITH $1-6$
SOXAXI ARRAY
SCUBOID VOID
\$CONCRETE REFLECTOR (SIDES)
SVOID
SVOID
SCONCRETE REFLECTOR (TOP)
SCONCRETE REFLECTOR (BOTTOM)
SZERO IMPORTANCE OUSSIDE WORLD

$1 \quad C Z 8.06$

CZ 8.3

$p Z$

pz +51.45

$p z+51.45$

$\mathrm{pz}+11.25$

px -15.25 


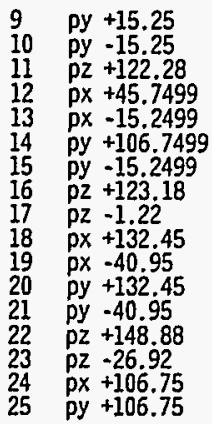

mode $n$

imp:n $1 \frac{1}{92} 11 \mathrm{r} 0$

$\begin{array}{ll}9234.50 \mathrm{c} & 9.45519 .6\end{array}$

92235.50c 8.58313-4

$92236.50 \mathrm{c} \quad 3.98110-6$

$92238.50 \mathrm{c} \quad 4.88625-5$

$7014.50 \mathrm{c} \quad 2.18930-3$

$8016.50 \mathrm{c} \quad 3.72979 .2$

$1001.50 \mathrm{C} \quad 5.81256-2$

mtl lwtr.01t

$12000.51 \mathrm{c} \quad 6.78153-4$

$13027.50 \mathrm{C} \quad 5.95056 .2$

$1400050 \mathrm{C} \quad 3.52126-4$

$2200051 \mathrm{c} \quad 3.03277-5$

$24000.51 \mathrm{C} \quad 1.03277 .5$

$24000.50 \mathrm{C}$ 5.30898-5

$25000.50 \mathrm{c}$

$29000.50 \mathrm{c}$

$1.38721-4$

$7014.50 \mathrm{c} \quad 1.99630-5$

$8016.50 \mathrm{c} \quad 4.23724-2$

$1001.50 \mathrm{C} \quad 1.04019-2$

$6012.50 \mathrm{C} \quad 6.42959-3$

$11023.50 \mathrm{C} \quad 3.83034-4$

$12000.51 \mathrm{C} \quad 7.18850-4$

$13027.50 \mathrm{C} \quad 1.12412-3$

$14000.50 \mathrm{C} \quad 7.71398-3$

$16032.51 \mathrm{c} \quad 8.28261-5$

$19000.51 \mathrm{C} \quad 4.89721-4$

20000.51c 8.02089-3

$22000.51 \mathrm{C} \quad 2.91932-5$

kcode 10001.010100

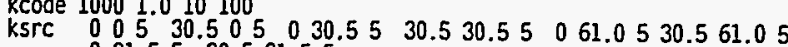

print

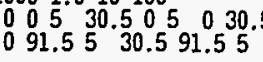

ROT64

$1-1.4984-1+3-5 \quad$

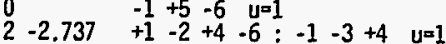

$+2:-4:+6 u=1$

$7.8+6$ प 10 (1)

$-12+13$ il

(12 $+13-14+15-16$

$+26-16+17$

$(+24:-8:+25:-26)-18+19-20+21+4-11$

$(+24:-8:+25:-26)-18+19-20+21+11-16$

$-2,3210$

(18 $-8,+25 ;-26)-18+19-20+21-4+17$

$\begin{array}{ll}-2.3210 & -18+19-20+21+16-22 \\ -2.3210 & -18+19-20+21-17+23\end{array}$

$+18:-19:+20:-21:+22=-23$

SCYLINDER OF URANYL NITRATE

SCYLINDER VOID

SCYLINDER OF ALUMINUM

SCELL FILLED WITH 1-6

$\$ 2 \times 3 \times 1$ ARRAY

5 CUBOID VOID

SCONCRETE REFLECTOR (SIDES)

SVOID

\$CONCRETE REFLECTOR (TOP)

SCONCRETE REFLECTOR (BOTTOH)

SZERO IMPORTANCE OUTSIDE WORLD

$\begin{array}{ll}1 & c z 8.06 \\ 2 & c z 8.38 \\ 3 & p z 0.0 \\ 4 & p z-0.32 \\ 5 & p z+65.49 \\ 6 & p z+119.1 \\ 7 & p x+15.25 \\ 8 & p x-15.25 \\ 9 & p y+15.25 \\ 10 & p y-15.25 \\ 11 & p z+122.28 \\ 12 & p x+45.7499 \\ 13 & p x-15.2499 \\ 14 & p y+76.2499 \\ 15 & p y-15.2499 \\ 16 & p z+123.18 \\ 17 & p z-1.22 \\ 18 & p x+132.45\end{array}$

January 1996

Final Report

Validation of MCNP4A on the Battelle

PSRM IBM RS/6000 Workstation 


$$
\begin{array}{ll}
19 & p x-40.95 \\
20 & p y+101.95 \\
21 & p y-71.45 \\
22 & p z+148.88 \\
23 & p z-26.92 \\
24 & p x+106.75 \\
25 & p y+76.25 \\
26 & p y-45.75
\end{array}
$$

mode $n$
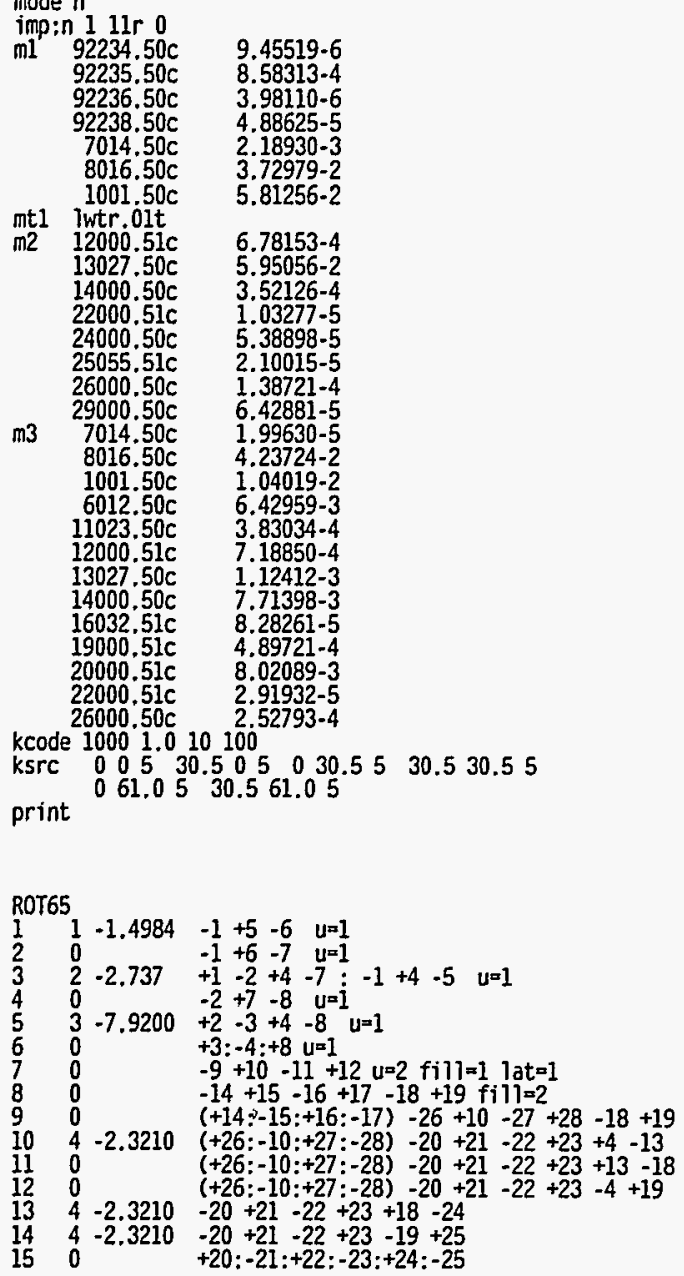

SCYLINDER OF URANYL NITRATE

SCYLINDER VOID

SCYLINDER OF ALUMINUM

SVOIO

SSTAIMLSS STEEL SLEEVE

SSTAIDLESS STEEL SLEEVE

SVOID

SCELL FILLED WITH 1-6

$\$ 2 \times 2 \times 1$ ARRAY

SCUBOID VOID

SCONCRETE REFLECTOR (SIDES)

SVOID

SVOID

SCONCRETE REFLECTOR (TOP)

SCONCRETE REFLECTOR (BOTTOM)

SZERO IMPORTANCE OUTSIDE WORLD
28.06
CZ 8.38
C2 8.69
pz -0.32
pz +101.45
$\mathrm{pz}+101.45$
$\mathrm{pz}+121.68$
$\mathrm{px}+15.25$
py +15.25
py -15.25
pz +122.28
px +45.7499
$p x-15.2499$
y +45.7499
py -15.2499
$\mathrm{pz}+123.18$
pz -1.22
$p x+132.45$
$p x-40.95$
py -101.95
py -101.95
$\mathrm{pz}-26,92$ 

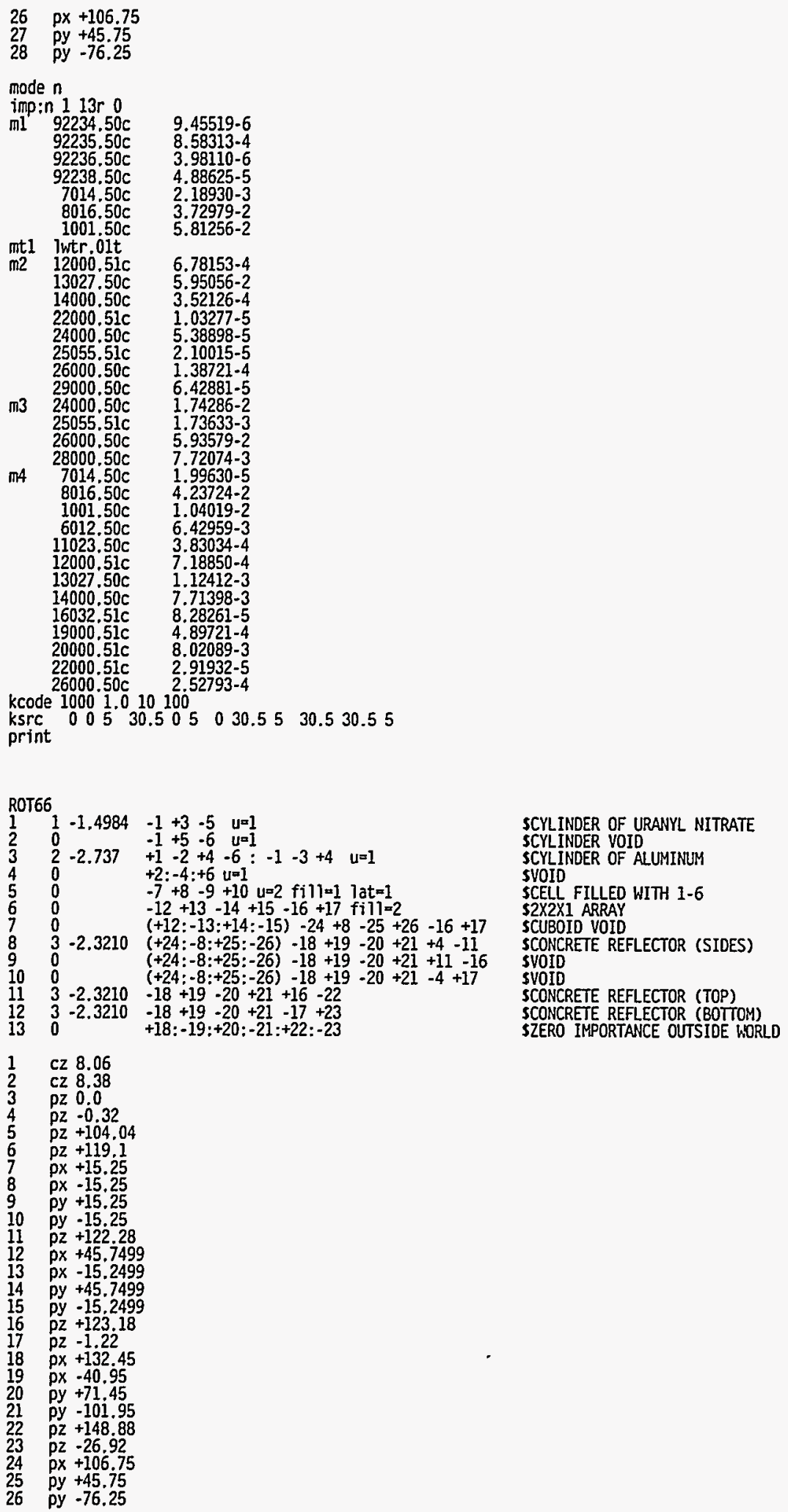

mode $n$

Imp: 1 IIr 0 
m1

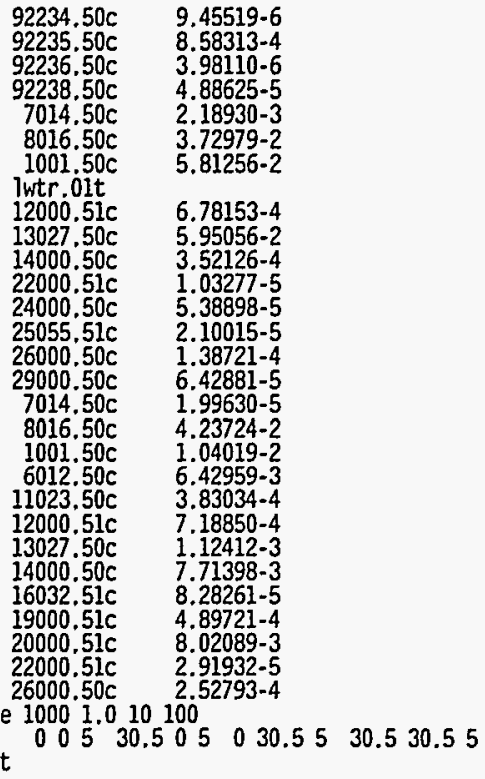

ROT67 BROMINE LEFT OUT OF PLEXIGLASS (M5)

1
2
3
4
5
6
7
8
9
10
11
12
SCYLINDER OF URANYL MITRATE

\section{SCYLINDER YOID}

SCYLINDER OF ALUMINUM

\$VOID

\$STAINLESS STEEL SLEEVE

SCELL FÍLLED WITH 1-6

$\$ 4 \times 4 \times 1$ ARRAY

SPLEXIGLASS REFLECTOR (SIOES)

SPLEXIGLASS REFLECTOR (TOP)

SPLEXIGLASS REFLECTOR (BOTTOM)

SZERO IMPORTANCE OUTSIDE WORLD

\footnotetext{
$1 \quad c z 10.56$

CZ 10.96

cz -0.3201

$\mathrm{pz}-0.3201$

$\mathrm{pz} 0.04 .82$

$p z+34.82$

$\mathrm{pz}+121.6801$

$\mathrm{px}+15.25$

$p x-15.25$

py +15.25

py -15.25

px +106.7499

px -15.2499

px -15.2499

py +106.7499

py -15.2499

$p z+121.68$

$\mathrm{pz}=32.55$

$\mathrm{px}+127.55$
$\mathrm{px}=36.05$

py +127.55

py -36.05

$p z+141.88$

$p z-21.12$
}

mode $n$

1mp: 11 10r 0

92234.50c $1.58625-6$

$92235.50 \mathrm{c} \quad 1.43995 .4$

$92236.50 \mathrm{c}$

(2)

$7014.50 \mathrm{c}$

$8016.50 \mathrm{c}$

$1001.50 \mathrm{c}$

mtl lwtr.01t

$\begin{aligned} & 3.40819-2 \\ & 6.53523-2\end{aligned}$

8.19743-6

. $50 \mathrm{c}$

6.78153-4

.95056-2 

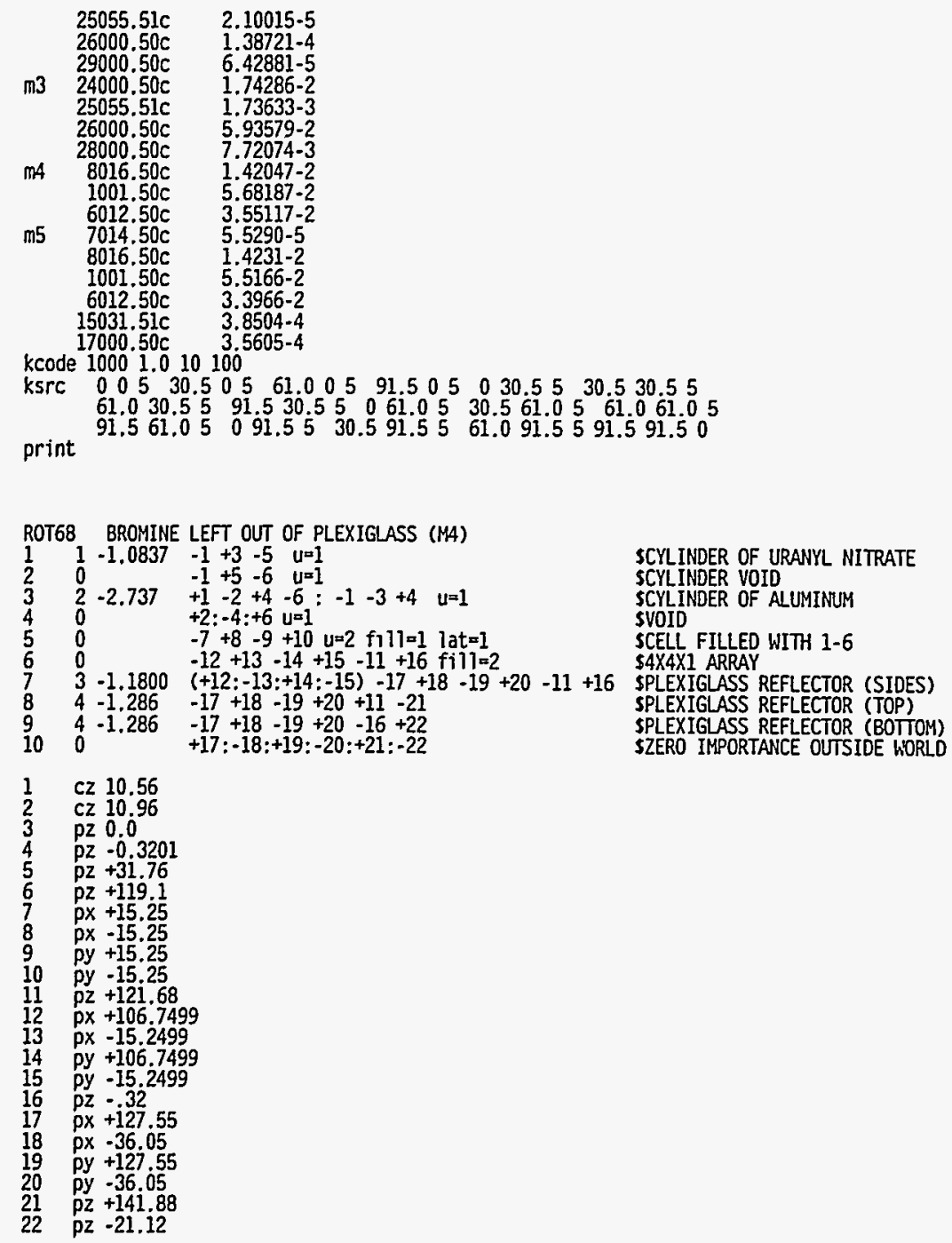

mode $n$

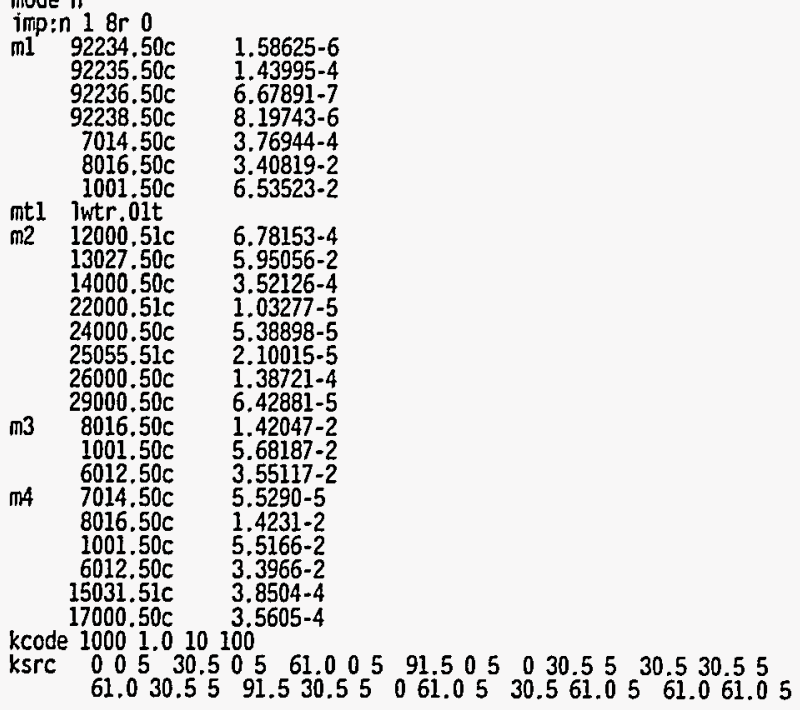

January 1996

Final Report

Validation of MCNP4A on the Battelle

Page 91 PSRM IBM RS/6000 Workstation 
$91.561 .05091 .55 \quad 30.591 .5561 .091 .5591 .591 .50$

print

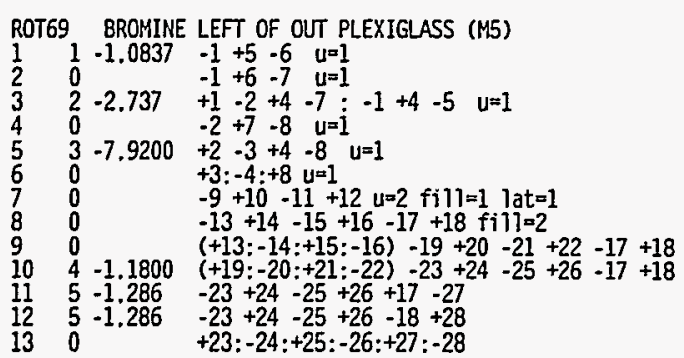

SCYLINDER OF URANYL NITRATE

SCYLINDER VOID

SCYLINDER OF ALUMINUM

SVOID

SSTAINLESS STEEL SLEEVE

SVOID

SCELL FILLED WITH 1-6

\$2X2X1 ARRAY

5 SUBOID VOID

SPLEXIGLASS REFLECTOR (SIDES)

SPLEXIGLASS REFLECTOR (TOP)

SPLEXIGLASS REFLECTOR (BOTTOM)

SZERO IMPORTANCE OUTSIDE WORLD

$\begin{array}{ll}1 & c z 10.56 \\ 2 & c z 10.96 \\ 3 & c z 11.28 \\ 4 & p z-0.3201 \\ 5 & p z-0.0 \\ 6 & p z+110.2 \\ 7 & p z+119.1 \\ 8 & p z+121.6801 \\ 9 & p x+15.25 \\ 10 & p x-15.25 \\ 11 & p y+15.25 \\ 12 & p y-15.25 \\ 13 & p x+45.7499 \\ 14 & p x-15.2499 \\ 15 & p y+45.7499 \\ 16 & p y-15.2499 \\ 17 & p z+121.68 \\ 18 & p z-32 \\ 19 & p x+76.25 \\ 20 & p x-45.75 \\ 21 & p y+76.25 \\ 22 & p y-45.75 \\ 23 & p x+97.05 \\ 24 & p x-66.55 \\ 25 & p y+97.05 \\ 26 & p y-66.55 \\ 27 & p z+141.88 \\ 28 & p z-21.12\end{array}$

mode $n$

imp:n 11110

$92234.50 \mathrm{c} \quad 1.58625-6$

$92235.50 \mathrm{c}$

$92238.50 \mathrm{c}$

$714.50 \mathrm{c}$

$8016.50 \mathrm{c}$

$1001.50 \mathrm{c}$

1.43995 .4

$6.67891-7$

$8.19743-6$

$3.70819-2$

$3.40819-2$
$6.53523-2$

mt1 lwtr. $01 \mathrm{t}$

$12000.51 \mathrm{c} \quad 6.78153-4$

$14000.50 \mathrm{C}$

1.03277-5

$24000.50 \mathrm{C}$

$25055.51 \mathrm{c}$

$26000.50 \mathrm{C}$

5.38898-5

$2.10015-5$

m3

$1.38721-4$

$24000.50 \mathrm{C} \quad 1.74286-2$

$25055.51 \mathrm{C} \quad 1.73633-3$

$26000.50 \mathrm{c} \quad 5.93579-2$

m4 $\begin{array}{rr}28000.50 \mathrm{c} & 7.72074-3 \\ 8016.50 \mathrm{c} & 1.42047-2\end{array}$

$1001.50 \mathrm{C} \quad 5.68187-2$

$6012.50 \mathrm{c} \quad 3.55117-2$

m5 $\quad 7014.50 \mathrm{c} \quad 5.5290-5$

$8016.50 \mathrm{C} \quad 1.4231-2$

$1001.50 \mathrm{c} \quad 5.5166-2$

$6012.50 \mathrm{c} 3.5966-2$

$\begin{array}{rr}6012.50 \mathrm{c} & 3.3966-2 \\ 15031.51 \mathrm{c} & 3.8504-4\end{array}$

$17000.50 \mathrm{C} \quad 3.5605-4$

kcode 10001010100

ksrc $005 \quad 30.505 \quad 0 \quad 30.55 \quad 30.530 .55$

print

ROT7O BROMINE LEFT OUT OF PLEXIGLASS (M4)

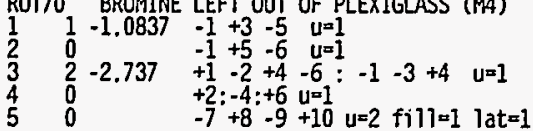

SCYLINDER OF URANYL NITRATE

SYLINOER VOID

SCYLINDER OF ALUMINUM

SCELL FILLED WITH 1-6
Final Report

January 1996 


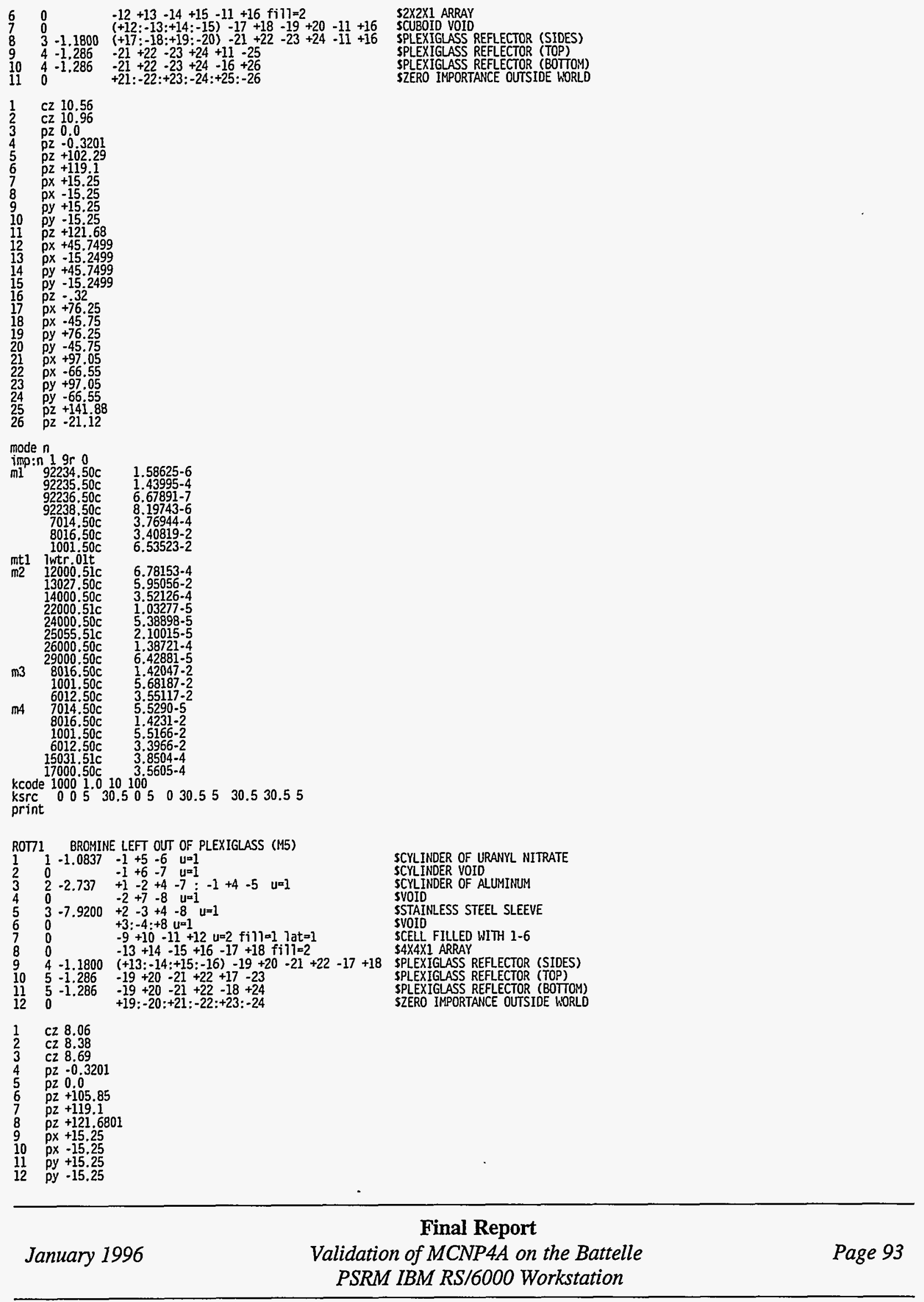




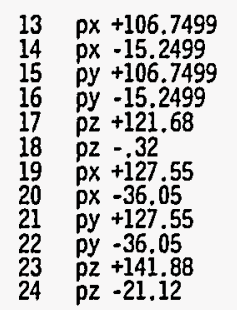

mode $n$

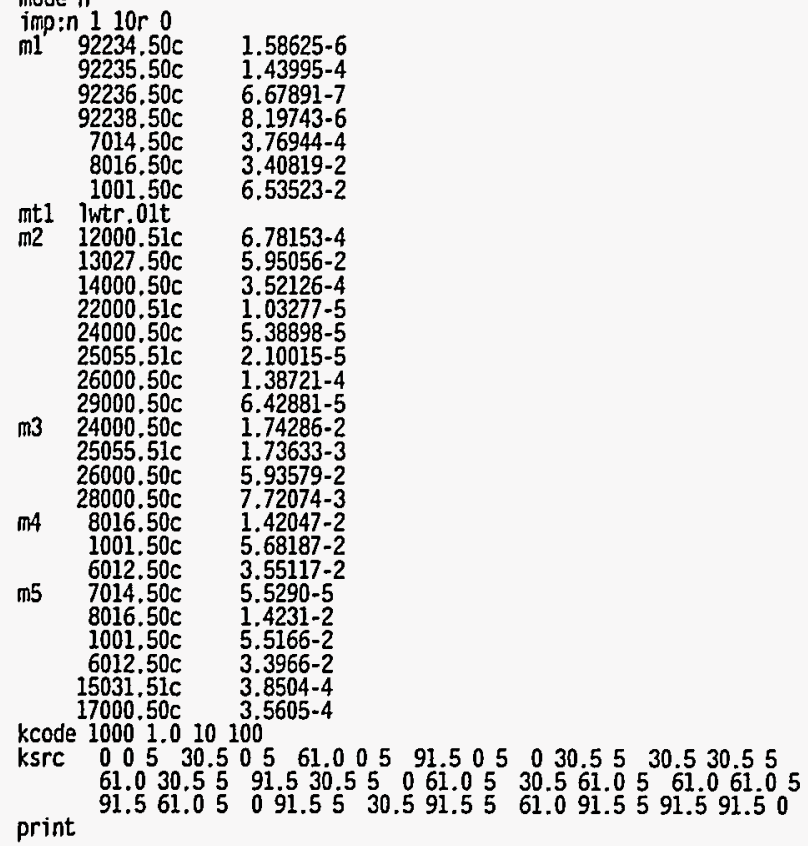

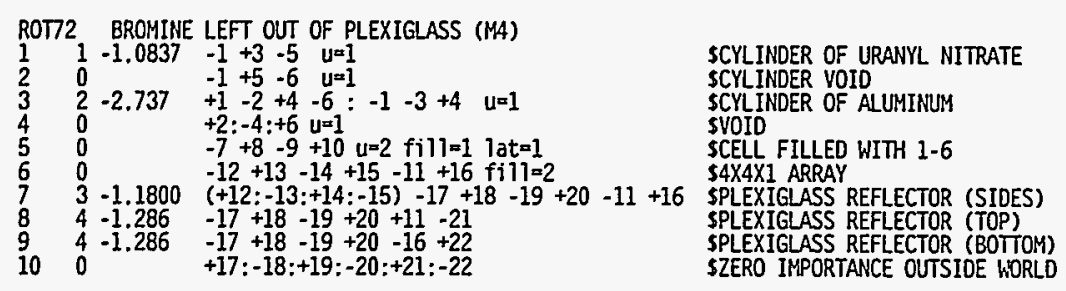

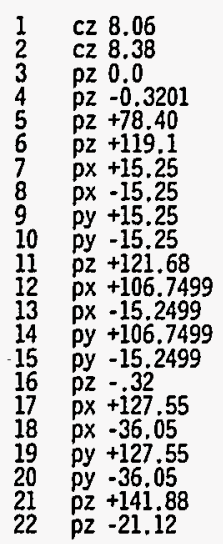

mode $n$

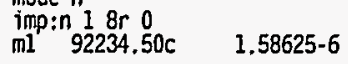




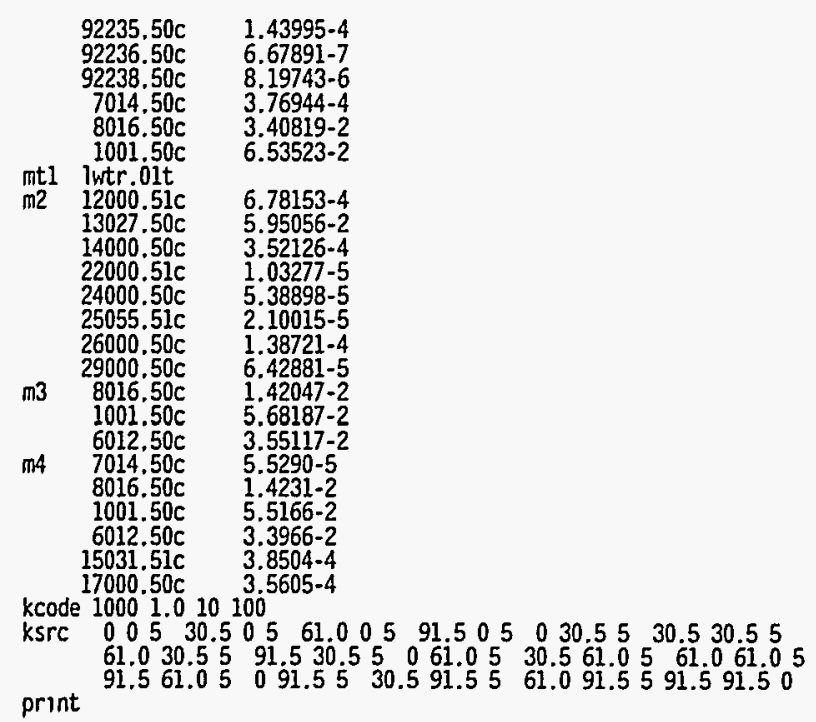
ROT73 BROHINE LEFT OUT OF PLEXIGLASS (M5)
$1 \quad 1-1.4925-1+5-6$,

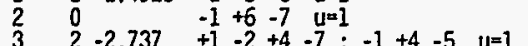

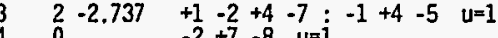

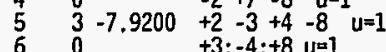
$7 \quad 0 \quad-9+10-11+12 u=2$ fill-1 lat $=1$
$80 \quad-13+14-15+16-17+18$ fill $=2$
$9 \quad 4-1.1800 \quad(+13:-14:+15:-16)-19+20-21+22-17+18$
$10 \quad 5-1.286-19+20-21+22+17-23$
I1 $5-1.286-19+20-21+22-18+24$
$120+19:-20:+21:-22:+23:-24 \quad$ SZERO IMPORTANCE OUTSIDE WORLD
SCYLINDER OF URANYL NITRATE
SCYLINDER VOID
SCYLINDER OF ALUMINUM
SVOID
\$STAINLESS STEEL SLEEVE
$\$ 4 \times 4 \times 1$ ARRAY

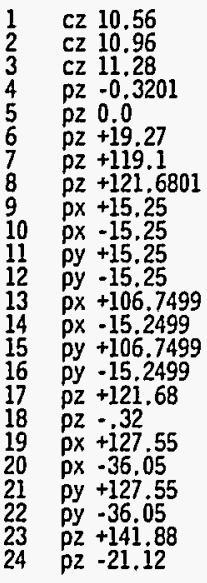

made $n$

Imp: $n 110 \mathrm{r} \quad 0$

$\mathrm{ml} 92234.50 \mathrm{c}$

$92235.50 \mathrm{C}$

$92236.50 \mathrm{c}$

$92238,50 \mathrm{c}$

$7014.50 \mathrm{c}$

$8016.50 \mathrm{c}$

$1001.50 \mathrm{c}$

mt1 lwtr.01t

$13027.50 \mathrm{c}$

$14000.50 \mathrm{c}$

$22000.51 \mathrm{C}$

$24000.50 \mathrm{c}$

$25055.51 \mathrm{c}$

$26000.50 \mathrm{c}$

$29000.50 \mathrm{c}$

m3 $24000.50 \mathrm{c}$

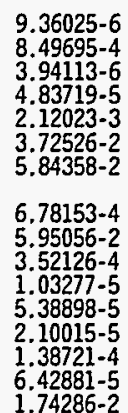

Final Report

January 1996 


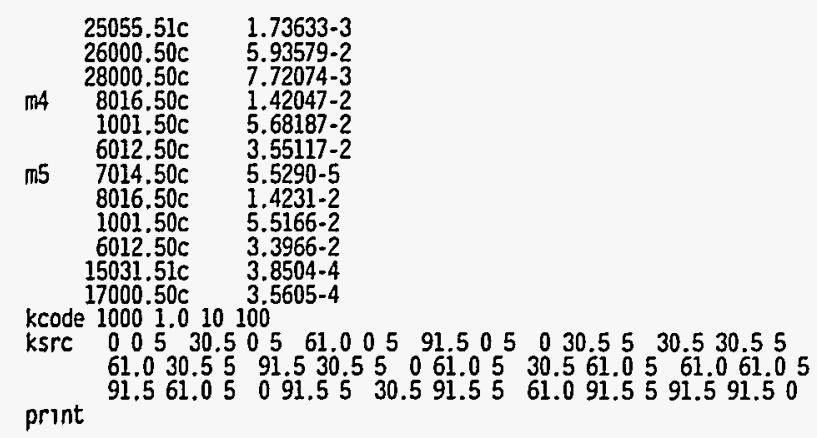

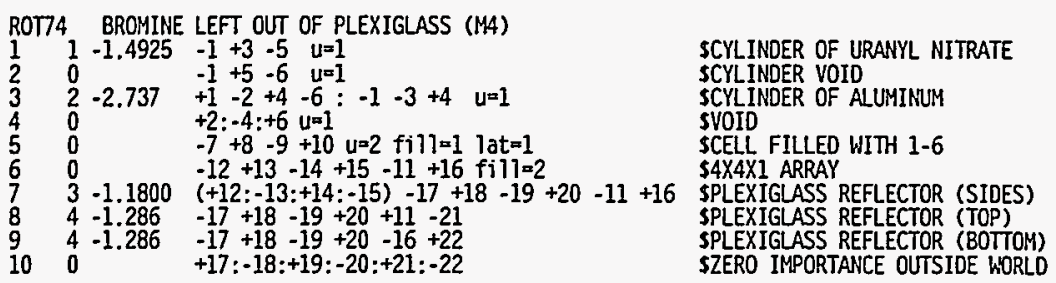

$\begin{array}{ll}1 & c z 10.56 \\ 2 & c z 10.96 \\ 3 & p z 0.0 \\ 4 & p z-0.3201 \\ 5 & p z+18.82 \\ 6 & p z+119.1 \\ 7 & p x+15.25 \\ 8 & p x-15.25 \\ 9 & p y+15.25 \\ 10 & p y-15.25 \\ 11 & p z+121.68 \\ 12 & p x+106.7499 \\ 13 & p x-15.2499 \\ 14 & p y+106.749 \\ 15 & p y-15.2499 \\ 16 & p z-32 \\ 17 & p x+127.55 \\ 18 & p x-36.05 \\ 19 & p y+127.55 \\ 20 & p y-36.05 \\ 21 & p z+141.88 \\ 22 & p z-21.12\end{array}$

mode $n$

Imp:n $18 r 0$

mip:n 18 8 0

$92235.50 \mathrm{c} \quad 9.36025-6$

92236.50c 8.49695-4

$92238.50 \mathrm{c} \quad 4.83719-5$

$7014.50 \mathrm{C} \quad 2.12023-3$

$8016.50 \mathrm{C} \quad 3.72526-2$

mtl $1001.50 \mathrm{c} \quad 5.84358-2$

$12000.51 \mathrm{C} \quad 6.78153-4$

$13027.50 \mathrm{C}$

$3.52126-4$

1.03277-5

$24000.50 \mathrm{C} \quad 5.38898-5$

25055.51c $2.10015-5$

$26000.50 \mathrm{C} \quad 1.38721-4$

m3 $8016.50 \mathrm{c} \quad 1.42047-2$

$1001.50 \mathrm{c} \quad 1.42047-2$

$1001.50 \mathrm{C} \quad 5.68187-2$

$\begin{array}{lll} & 6012.50 \mathrm{c} & 3.55117-2 \\ & 7014.50 \mathrm{c} & 5.5290-5\end{array}$

$\begin{array}{ll}7014.50 \mathrm{C} & 5.5290-5 \\ 8016.50 \mathrm{C} & 1.4231-2\end{array}$

$1001.50 \mathrm{c} \quad 5.5166 .2$

$6012.50 \mathrm{c}$

$15031.51 \mathrm{C} \quad 3.8504$

kcode 10001.010100

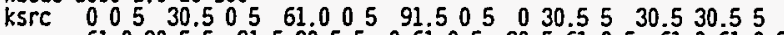

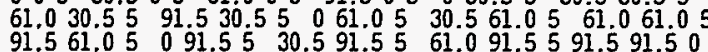

print

ROT75 BROMINE LEFT OUT OF PLEXIGLASS (M5) 

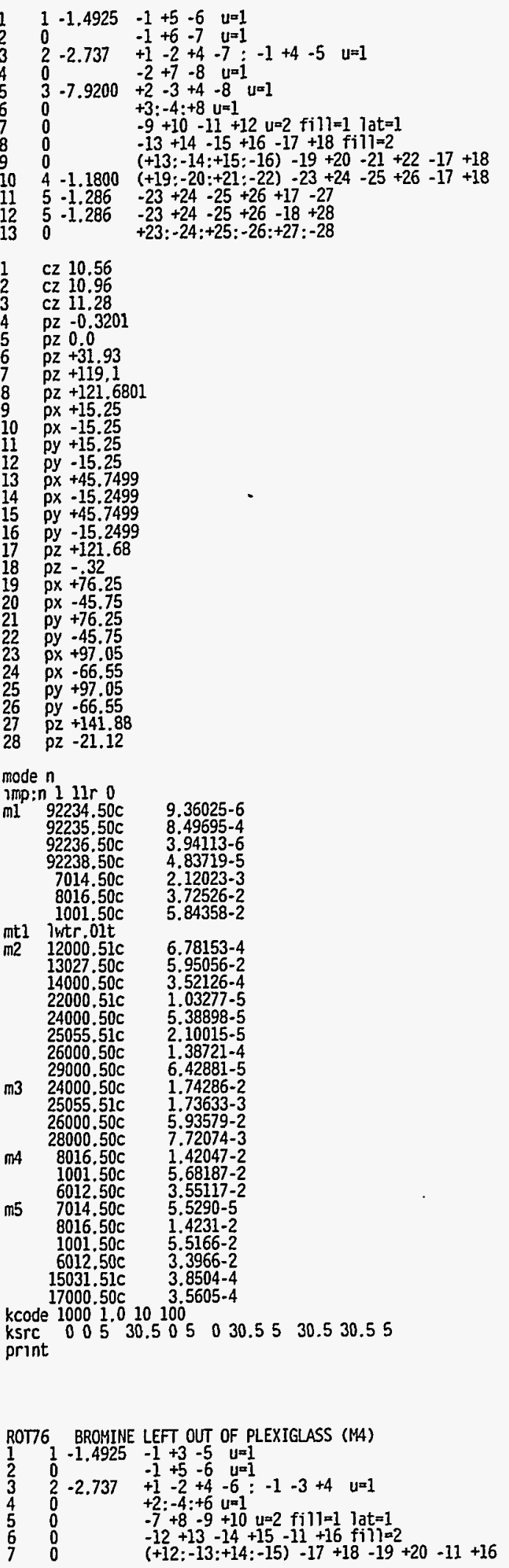

SCYLINDER OF URANYL NITRATE

SCYLINDER VOID

SCYLINDER OF ALUMINUM

SVOID

SSTAINLESS STEEL SLEEVE

SVOIO

SCELL FILLED WITH 1-6

$\$ 2 \times 2 \times 1$ ARRAY

SPLEXIGLASS REFLECTOR (SIOES)
SPLEXIGLASS REFLECTOR (TOP)

SPLEXIGLASS REFLECTOR (BOTTOM)
SCYL INDER OF URANYL NITRATE

SCYLINDER VOID

SCYLINDER OF ALUMINUH

SCYLINOE

SVID FIUED WITH 1-6

STEL FILLEO WITH 1-6

\$2X2X1 ARRAY
SCUBOID VOID

Final Report

January 1996 


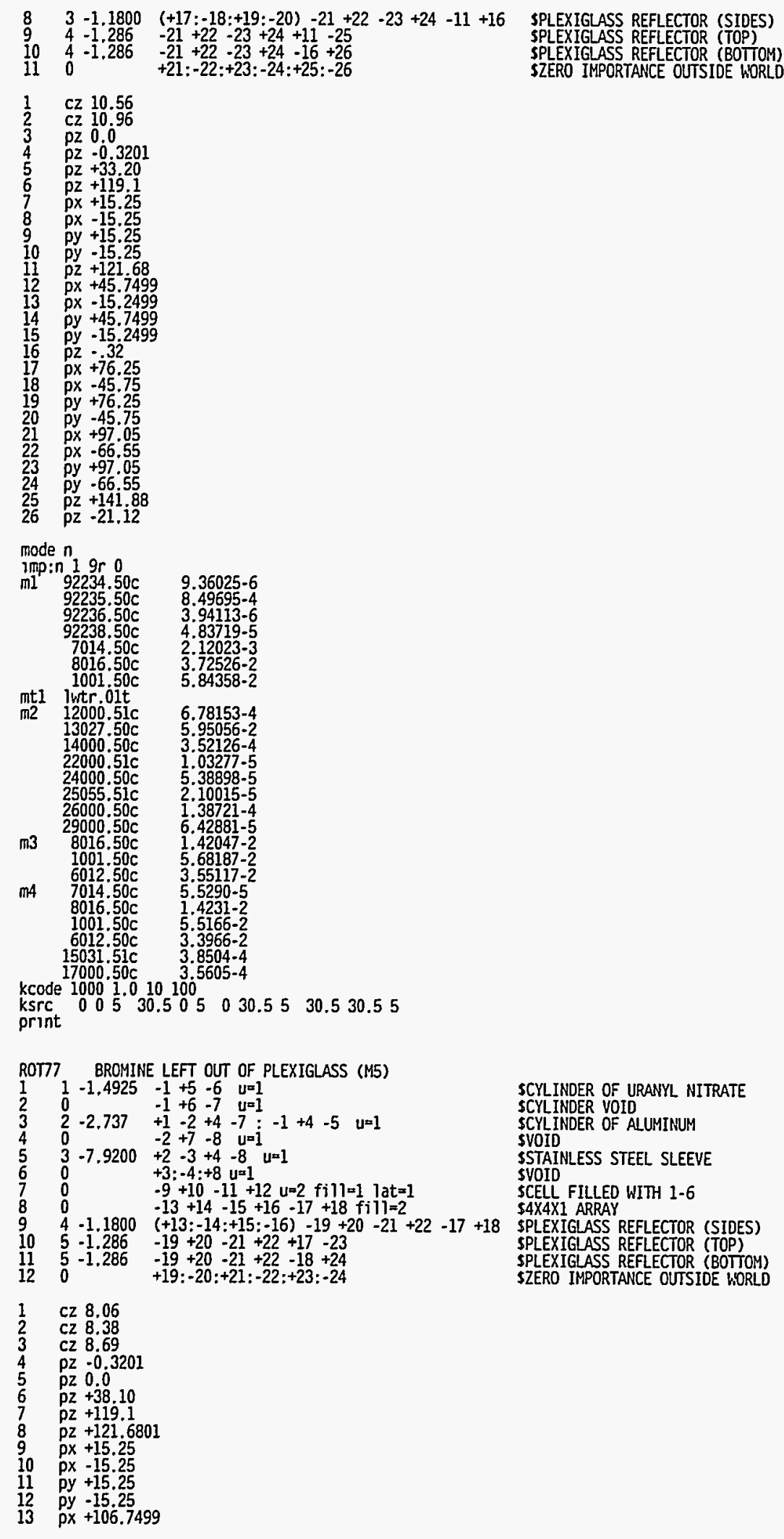



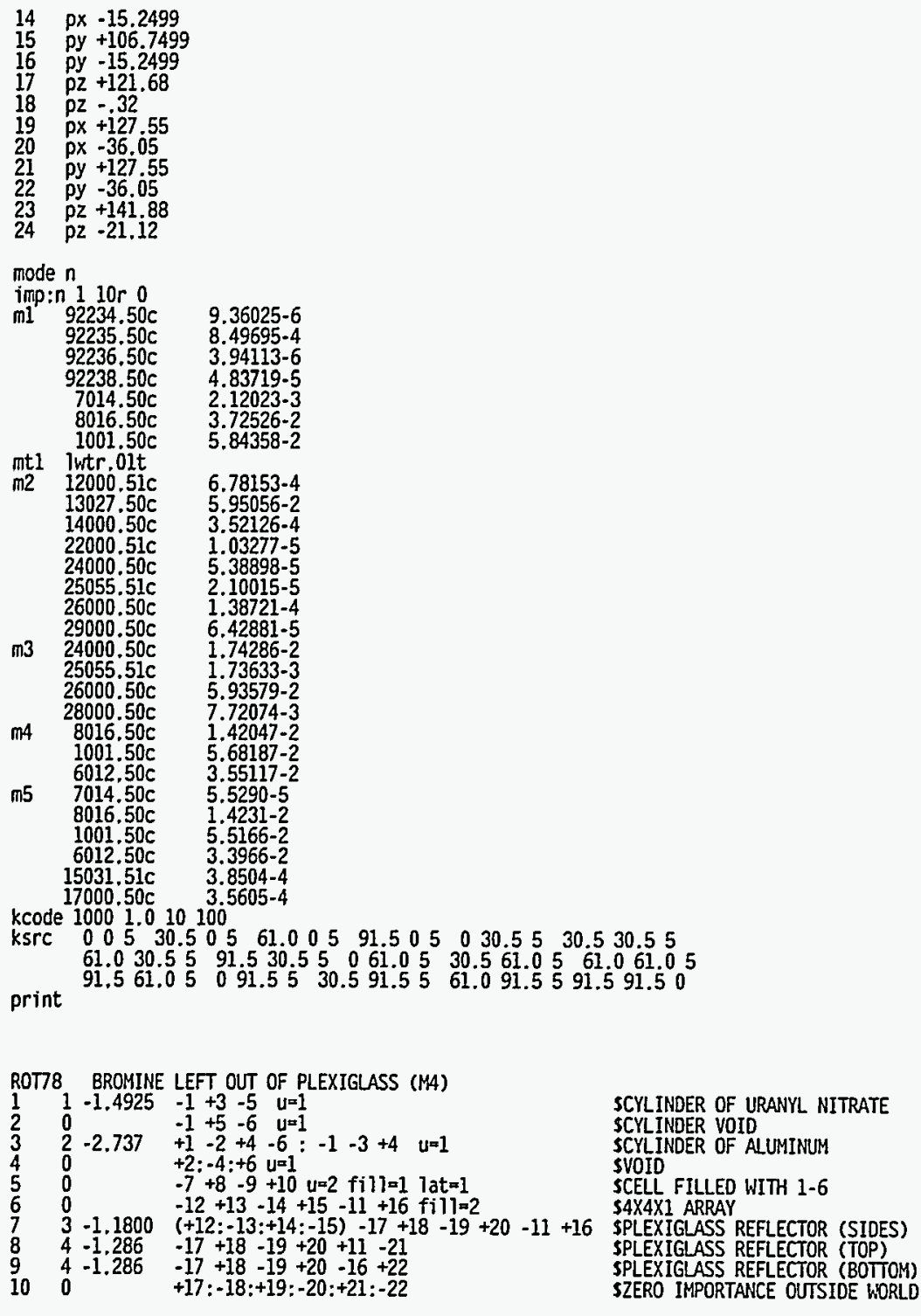
$1 \quad$ Cz 8.06
CZ 8.38
pz 0.0
pz $=0.3201$
pz +35.56
pz +119 .
$\mathrm{px}+15.25$
px -15.25
py +15.25
py -15.25
$p z+121.68$
$p x+106.7499$
PX -15.2499
py +106.7499
py -15.249
pz $=.32$
$\mathrm{px}+127.55$
px $-36,05$
py +127.55
py -36.05
pz +141.88
$\mathrm{pZ}-21,12$

mode

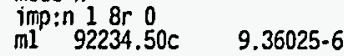




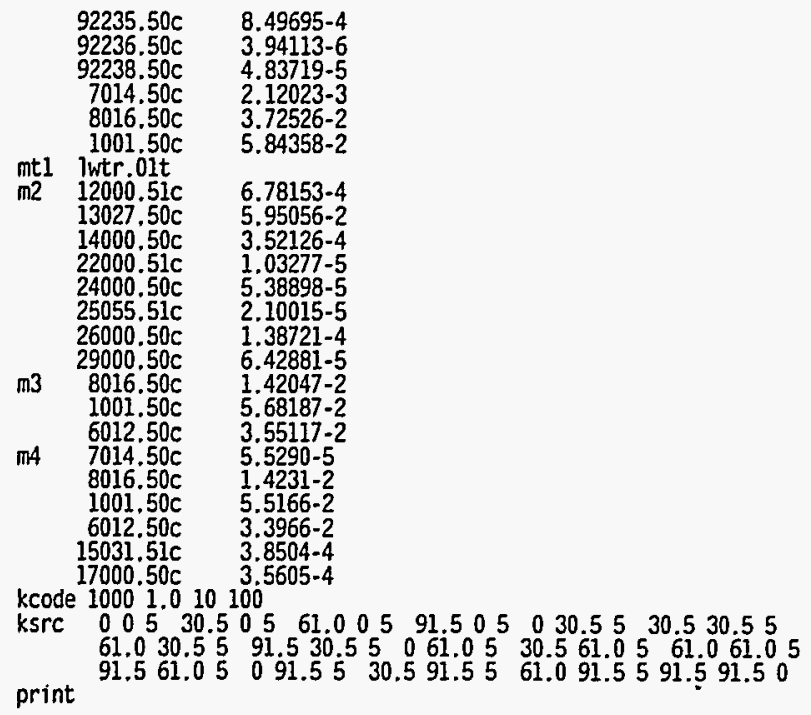

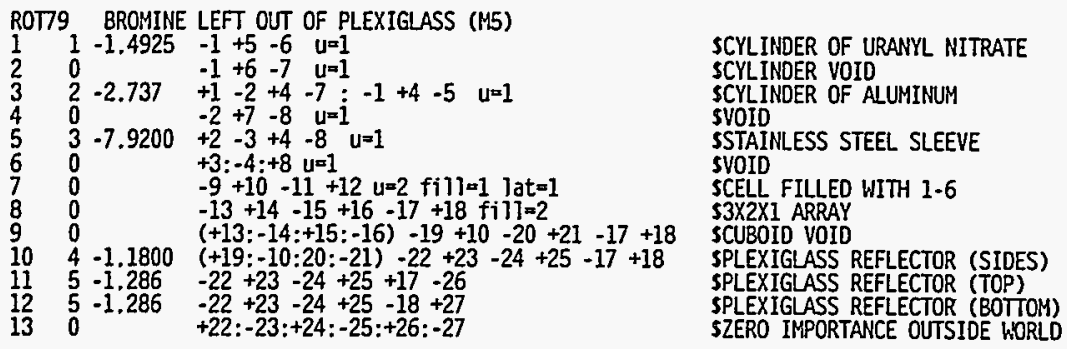

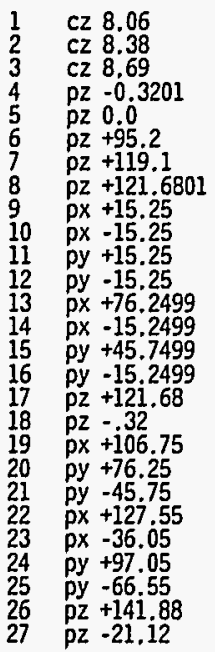

mode $n$
Imp: ${ }_{92234} 110$
$92235.50 \mathrm{c}$ $92236.50 \mathrm{c}$ ( $7230.50 \mathrm{c}$ $7014.50 \mathrm{C}$ $8016.50 \mathrm{C}$ $1001.50 \mathrm{C}$
mtl lwtr.01t $12000.51 \mathrm{c}$ $13027.50 \mathrm{C}$ $14000.50 \mathrm{c}$ (2) $24000.50 \mathrm{C}$

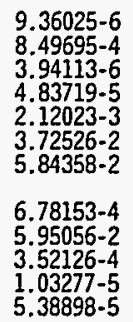



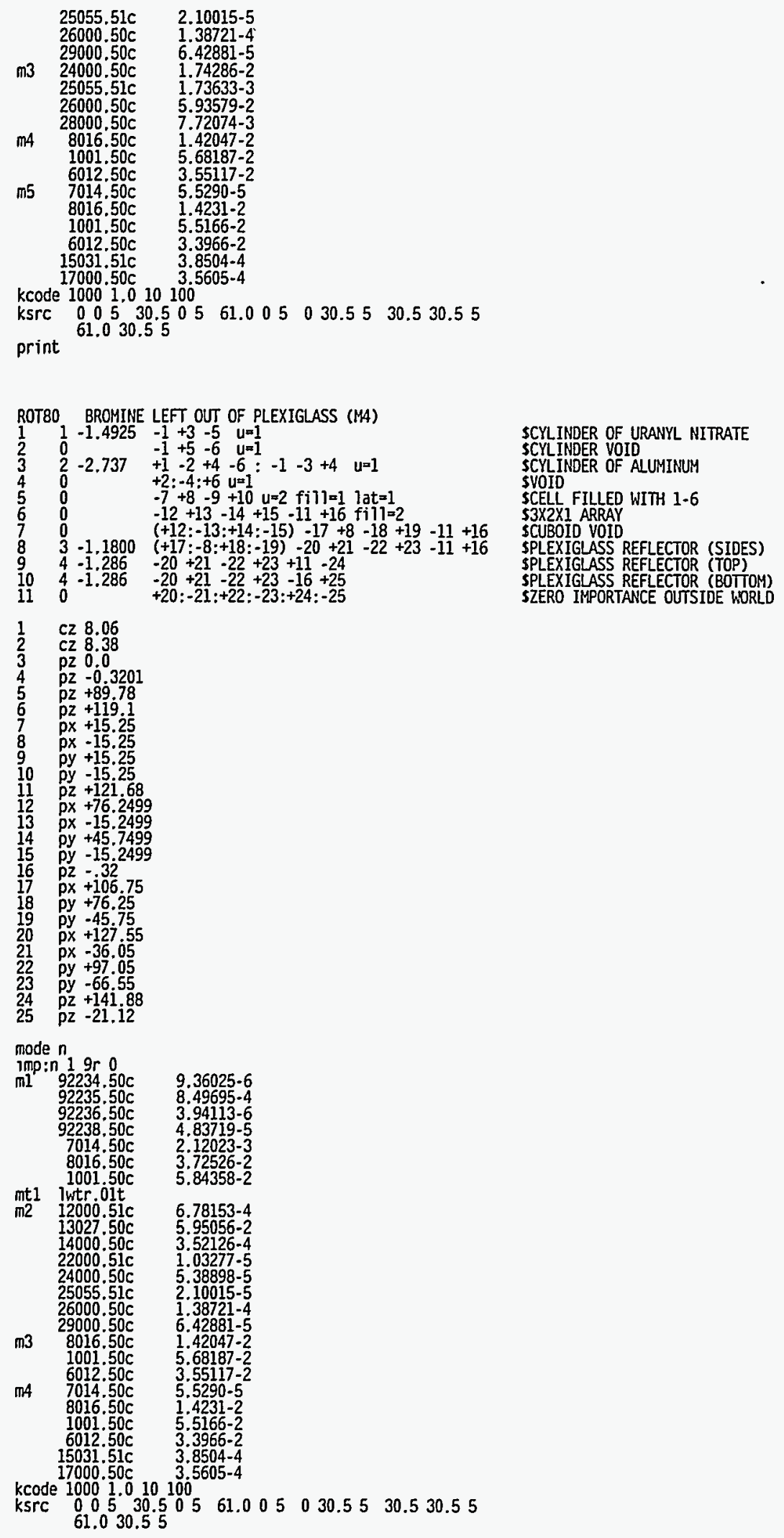

\section{Final Report}

January 1996 

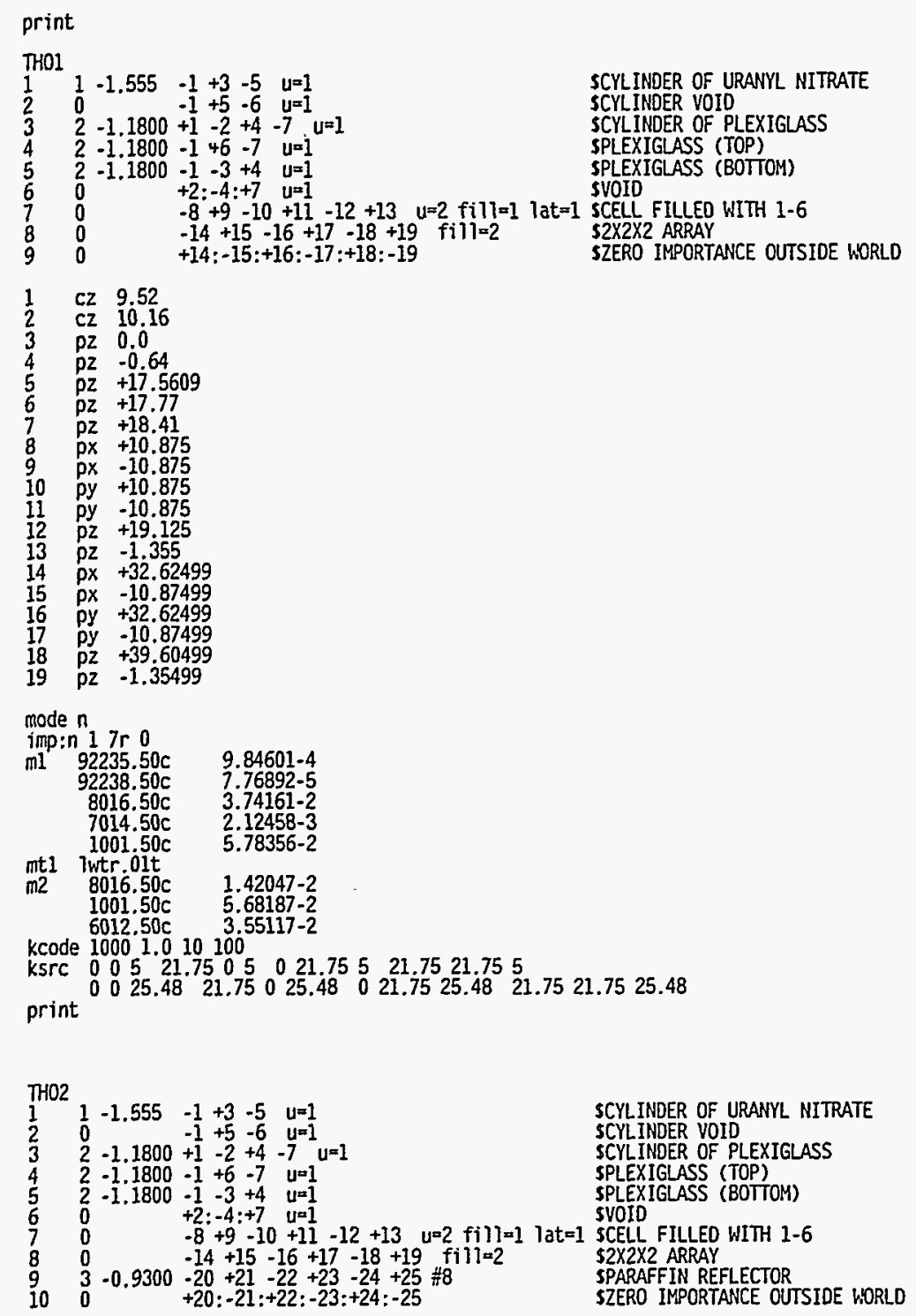

$\begin{array}{lll}1 & c z & 9.52 \\ 2 & c z & 10.16 \\ 3 & p z & 0.0 \\ 4 & p z & -0.64 \\ 5 & p z & +17.5609 \\ 6 & p z & +17.77 \\ 7 & p z & +18.41 \\ 8 & p x & +11.80 \\ 9 & p x & -11.80 \\ 10 & p y & +11.80 \\ 11 & p y & -11.80 \\ 12 & p z & +20.05 \\ 13 & p z & -2.28 \\ 14 & p x & +35.39999 \\ 15 & p x & -11.79999 \\ 16 & p y & +35.39999 \\ 17 & p y & -11.7999 \\ 18 & p z & +42.37999 \\ 19 & p z & -2.27999 \\ 20 & p x & +36.67 \\ 21 & p x & -13.07 \\ 22 & p y & +36.67 \\ 23 & p y & -13.07 \\ 24 & p z & +43.65 \\ 25 & p z & -3.55\end{array}$ 
mode $n$

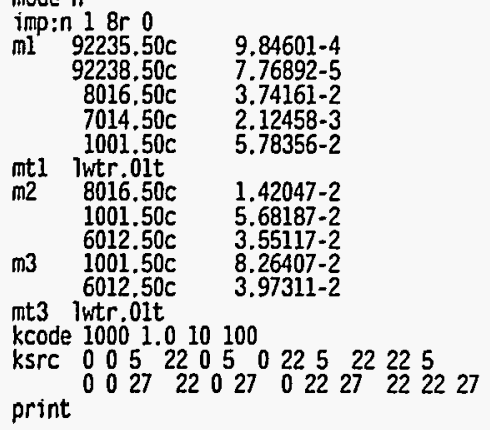

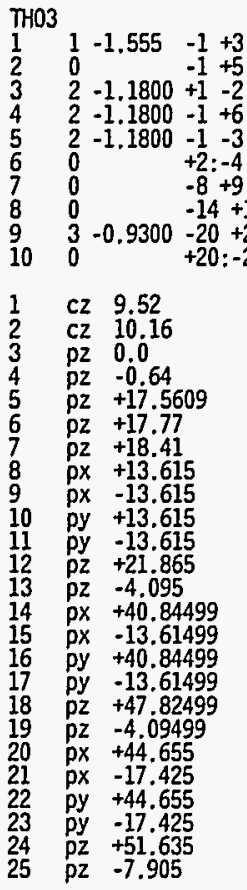

made $n$
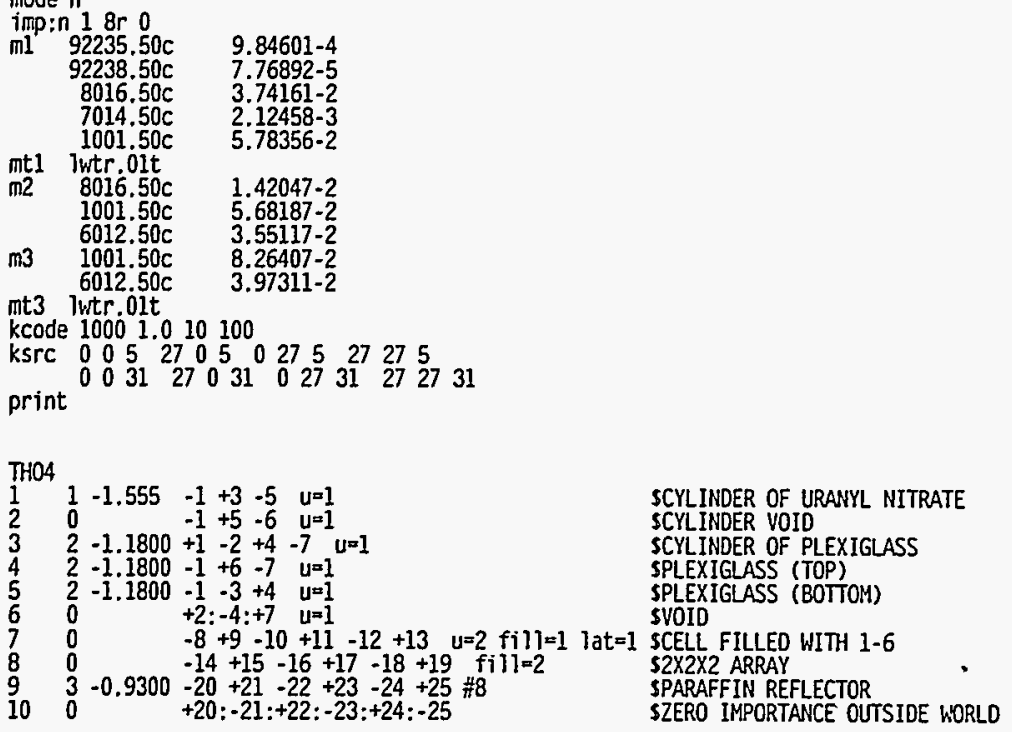

SCYLINDER OF URANYL NITRATE SCYLINDER VOID SCYLINDER OF PLEXIGLASS SPLEXIGLASS (TOP) SPLEXIGLASS (BOTTOM) SVOID

SCELL FILLED WITH 1-6

$\$ 2 \times 2 \times 2$ ARRAY

SPARAFFIN REFLECTOR

SZERO IMPORTANCE OUTSIDE WORLD

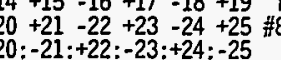




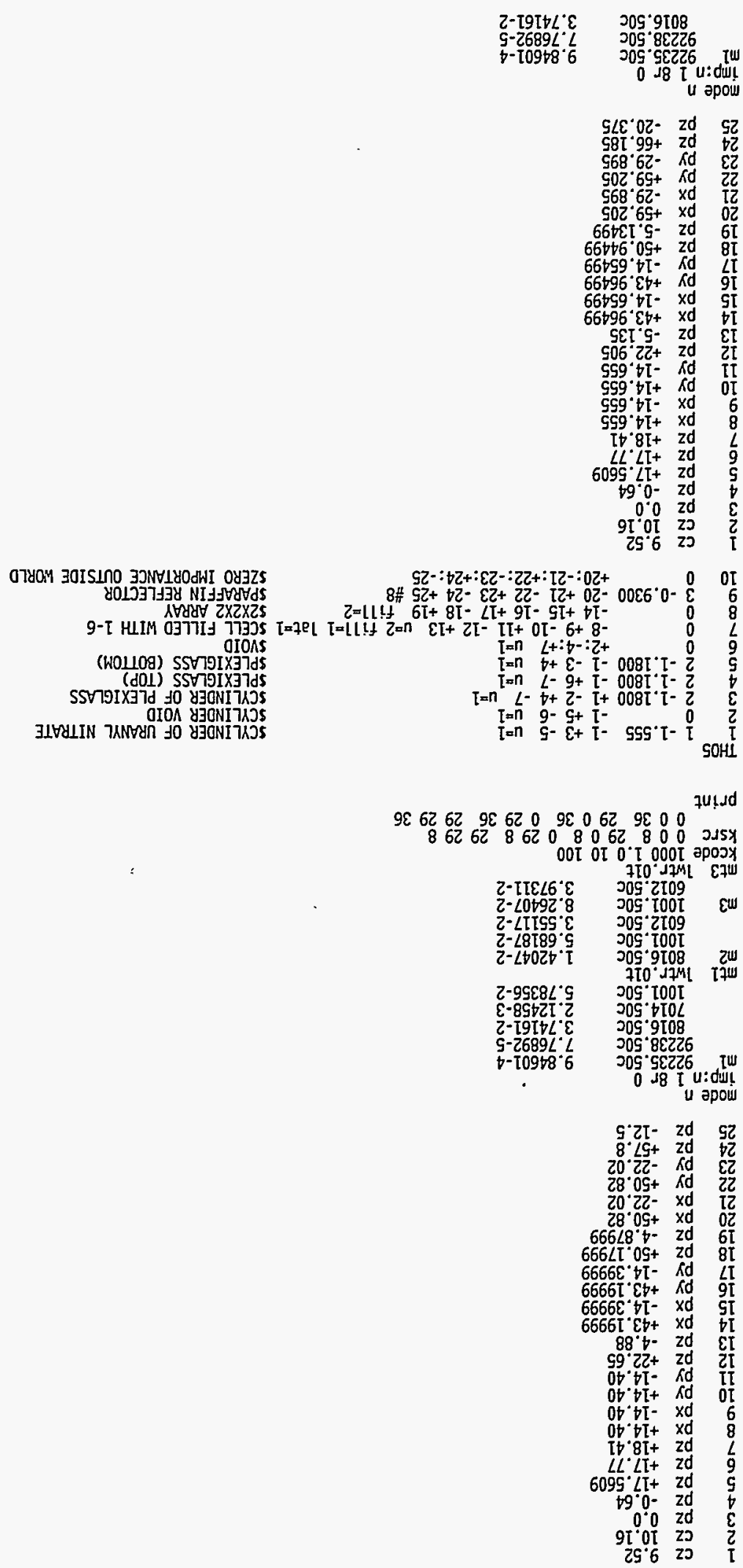




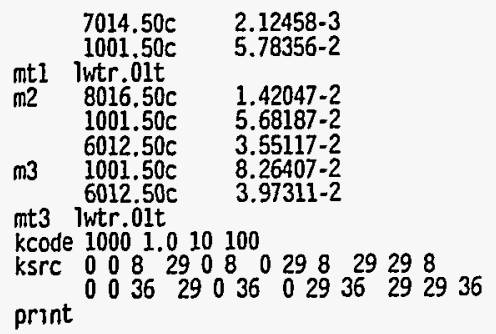

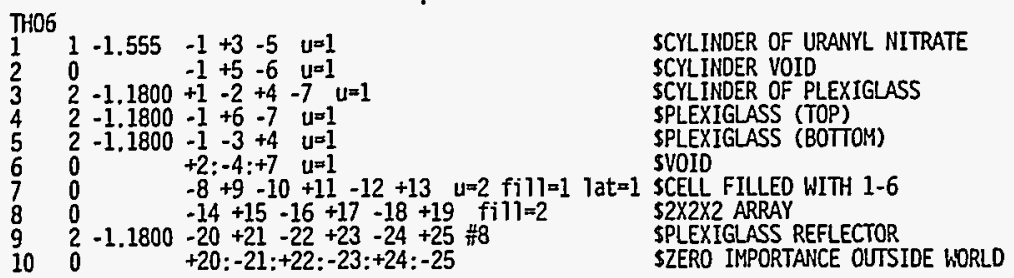

$\begin{array}{lll}1 & c z & 9.52 \\ 2 & c z & 10.16 \\ 3 & p z & 0.0 \\ 4 & p z & -0.64 \\ 5 & p z & +17.5609 \\ 6 & p z & +17.77 \\ 7 & p z & +18.41 \\ 8 & p x & +11.66 \\ 9 & p x & -11.66 \\ 10 & p y & +11.66 \\ 11 & p y & -11.66 \\ 12 & p z & +19.91 \\ 13 & p z & -2.14 \\ 14 & p x & +34.97999 \\ 15 & p x & -11.65999 \\ 16 & p y & +34.97999 \\ 17 & p y & -11.65999 \\ 18 & p z & +41.95999 \\ 19 & p z & -2.13999 \\ 20 & p x & +36.35 \\ 21 & p x & -12.93 \\ 22 & p y & +36.35 \\ 23 & p y & -12.93 \\ 24 & p z & +43.23 \\ 25 & p z & -3.41\end{array}$
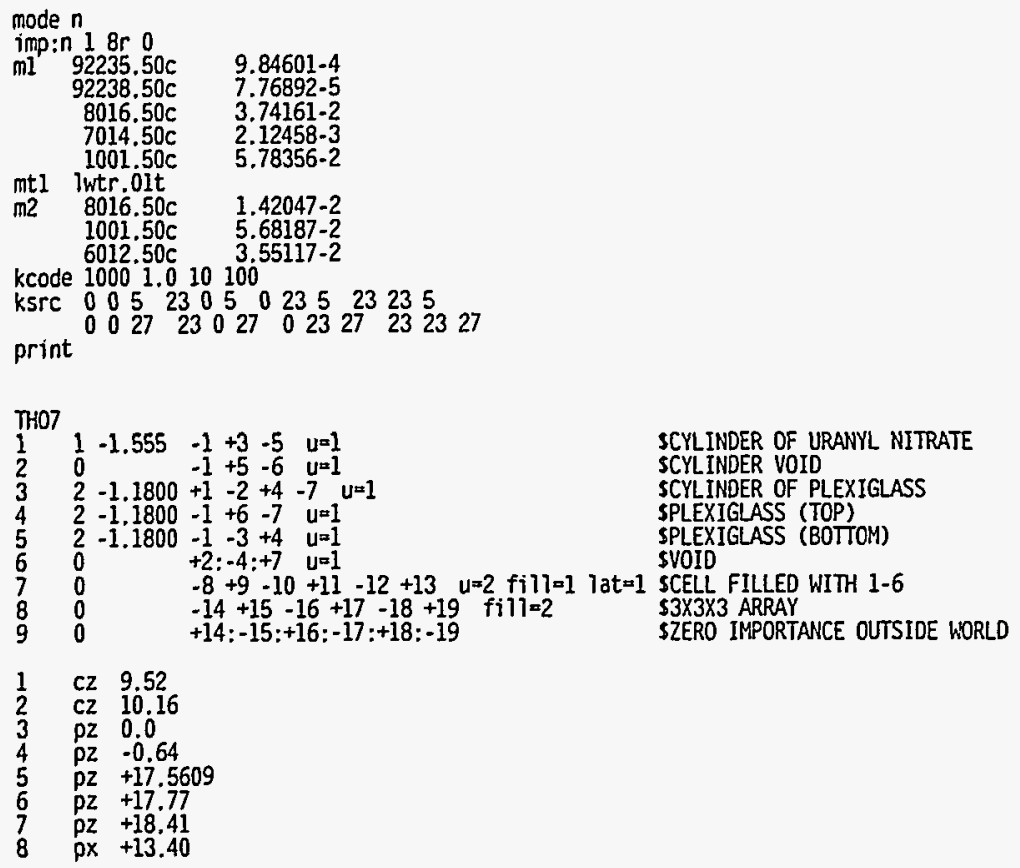

SCYLINDER OF URANYL NITRATE CYLINDER OF PLEXIGLASS SPLEXIGLASS (TOP) 


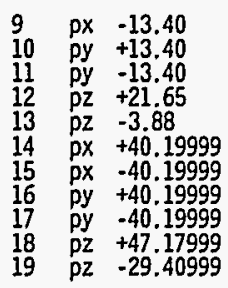

mode $n$

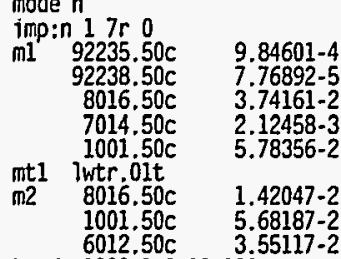

kcode 10001.010100

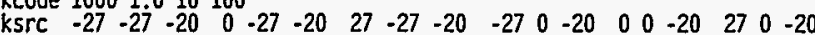

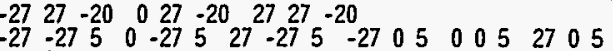

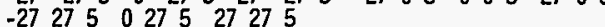

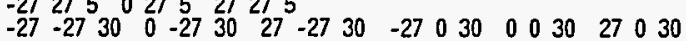

print

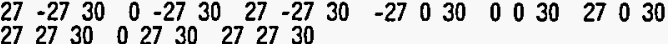

THOB

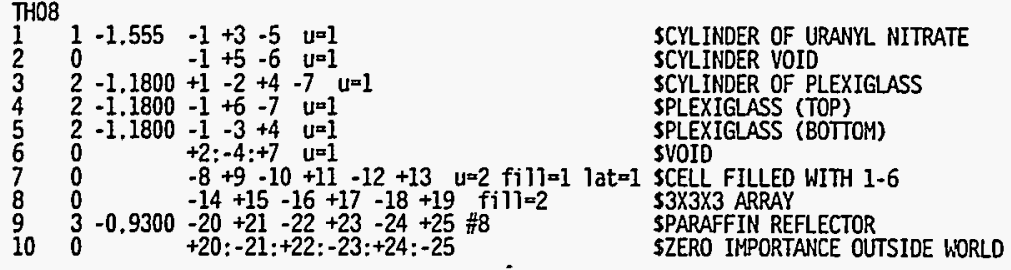

$\begin{array}{lll}1 & c z & 9.52 \\ 2 & c z & 10.16 \\ 3 & p z & 0.0 \\ 4 & p z & -0.64 \\ 5 & p z & +17.5609 \\ 6 & p z & +17.77 \\ 7 & p z & +18.41 \\ 8 & p x & +14.67 \\ 9 & p x & -14.67 \\ 10 & p y & +14.67 \\ 11 & p y & -14.67 \\ 12 & p z & +22.92 \\ 13 & p z & -5.15 \\ 14 & p x & +44.00999 \\ 15 & p x & -44.00999 \\ 16 & p y & +44.00999 \\ 17 & p y & -44.00999 \\ 18 & p z+50.98999 \\ 19 & p z & -33.21999 \\ 20 & p x & +45.28 \\ 21 & p x & -45.28 \\ 22 & p y & +45.28 \\ 23 & p y & -45.28 \\ 24 & p z+52.26 \\ 25 & p z & -34.49\end{array}$

mode $n$

$$
\begin{array}{ccc}
\text { imp:n } 18 \mathrm{r} 0 & \\
\mathrm{ml} & 92235.50 \mathrm{c} & 9.84601-4 \\
& 92238.50 \mathrm{c} & 7.76892-5 \\
& 8016.50 \mathrm{c} & 3.74161-2 \\
& 7014.50 \mathrm{c} & 2.12458-3 \\
& 1001.50 \mathrm{c} & 5.78356-2 \\
\mathrm{mt} 1 & 1 \mathrm{wtr} .01 \mathrm{t} & \\
\mathrm{m} 2 & 8016.50 \mathrm{c} & 1.42047-2 \\
& 1001.50 \mathrm{c} & 5.68187-2 \\
& 6012.50 \mathrm{c} & 3.55117-2 \\
\mathrm{~m} 3 & 1001.50 \mathrm{c} & 8.26407-2 \\
& 6012.50 \mathrm{c} & 3.97311-2
\end{array}
$$

mt3 Iwtr.01t

kcode 10001010100

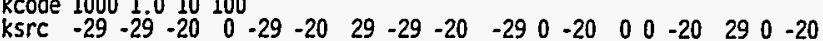

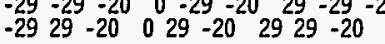




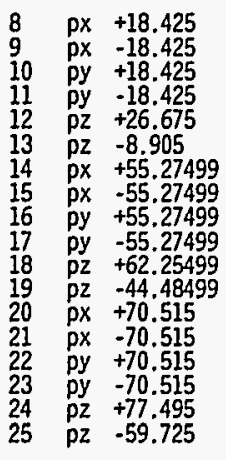

mode $n$

imp:n $18 r 0$

92235.50c $\quad 9.84601-4$

$92238.50 \mathrm{c} \quad 7.76892-5$

$7014.50 \mathrm{C} \quad 2.12458-3$

$1001.50 \mathrm{C} \quad 5.78356-2$

mi lintr

m2 $8016.50 \mathrm{C} \quad 1.42047-2$

$6012.50 \mathrm{c} \quad 3.55117-2$

$\mathrm{m} 3 \quad 1001.50 \mathrm{C} \quad 8.26407-2$

$6012.50 \mathrm{C} \quad 3.97311-2$

mt3 iwtr.01t

kcode 100011010100

$\begin{array}{lllllllllllllllllll}\text { ksrc } & -37 & -37 & -30 & 0 & -37 & -30 & 37 & -37 & -30 & -37 & 0 & -30 & 0 & 0 & -30 & 37 & 0 & -30\end{array}$

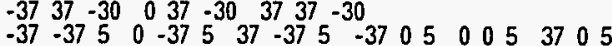

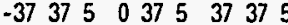

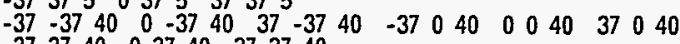

pront

THO11

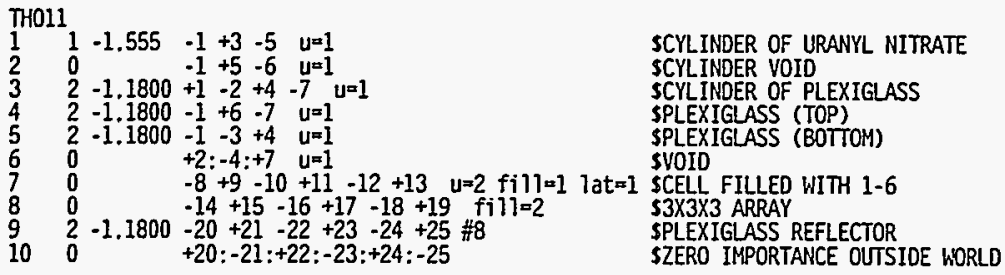

$\begin{array}{lll}1 & c z & 9.52 \\ 2 & c z & 10.16 \\ 3 & p z & 0.0 \\ 4 & p z & -0.64 \\ 5 & p z & +17.5609 \\ 6 & p z & +17.77 \\ 7 & p z & +18.41 \\ 8 & p x & +14.54 \\ 9 & p x & -14.54 \\ 10 & p y & +14.54 \\ 11 & p y & -14.54 \\ 12 & p z & +22.79 \\ 13 & p z & -5.02 \\ 14 & p x & +43.61999 \\ 15 & p x & -43.61999 \\ 16 & p y & +43.61999 \\ 17 & p y & -43.61999 \\ 18 & p z & +50.59999 \\ 19 & p z & -32.82999 \\ 20 & p x & +44.89 \\ 21 & p x & -44.89 \\ 22 & p y & +44.89 \\ 23 & p y & -44.89 \\ 24 & p z & +51.87 \\ 25 & p z & -34.10\end{array}$

mode $n$

imp: $n 18 r 0$

92235.50c

$2238.50 c$

$8016.50 \mathrm{c}$

$7014.50 \mathrm{C}$
$1001.50 \mathrm{C}$

mtl iwtr.olt

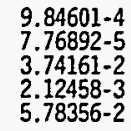

9.84601-4

.74161-2

$5.78356-2$ 


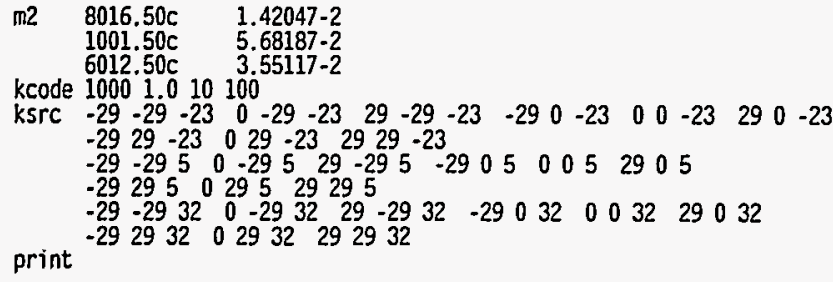

mode $n$

imp: 1 1 $7 r 0$

$\mathrm{ml}$ " $92235.50 \mathrm{c}$

$7.76892-5$

$1001.50 \mathrm{c} \quad 2.12458-3$

mi l intr 010

$8016.50 \mathrm{c} \quad 1.42047-2$

$5.68187-2$

kcode 10001010100

ksrc $0005 \quad 310506205930500315 \quad 31315 \quad 6231593315$

$062531625 \quad 6262593625 \quad 0935 \quad 319356293593935$

$\begin{array}{lllllllllllllll} & & 35 & 31 & 35\end{array}$

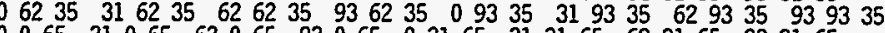

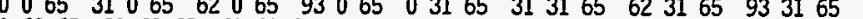

$\begin{array}{llllllllllllllllllllllllllll}0 & 62 & 65 & 31 & 62 & 65 & 62 & 62 & 65 & 93 & 62 & 65 & 0 & 93 & 65 & 31 & 93 & 65 & 62 & 93 & 65 & 93 & 93 & 65\end{array}$ print

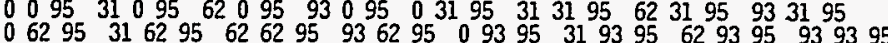

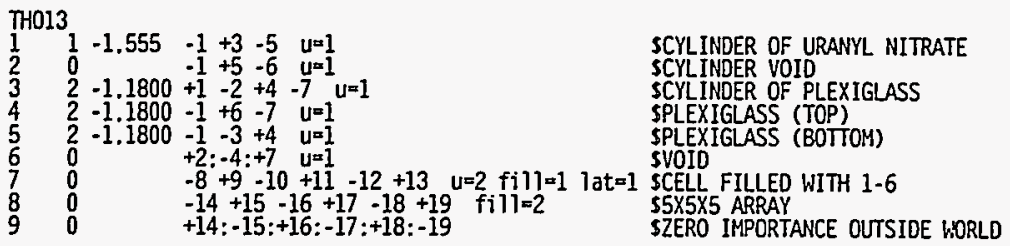

$\begin{array}{lll}1 & c z & 9.52 \\ 2 & c z & 10.16 \\ 3 & p z & 0.0 \\ 4 & p z & -0.64 \\ 5 & p z & +17.5609 \\ 6 & \rho z & +17.77 \\ 7 & p z & +18.41 \\ 8 & p x & +17.36 \\ 9 & p x & -17.36 \\ 10 & p y & +17.36 \\ 11 & p y & -17.36 \\ 12 & p z & +25.61\end{array}$

January 1996

Final Report

Validation of MCNP4A on the Battelle

PSRM IBM RS/6000 Workstation 

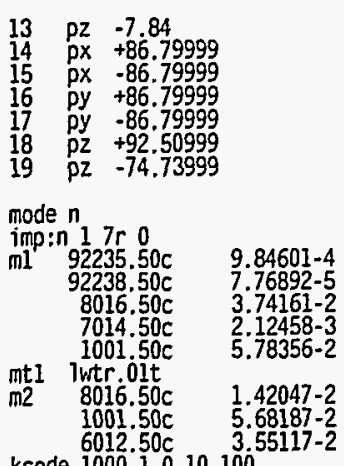

$6012.50 \mathrm{c} \quad 3.65117-2$

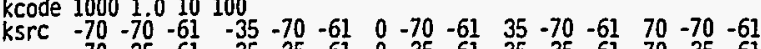

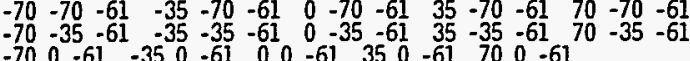

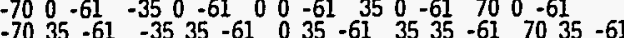

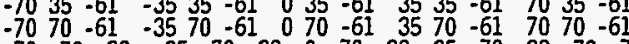

$\begin{array}{lllllllllllllll}-70 & -70 & -28 & -35 & -70 & -28 & 0 & -70 & -28 & 35 & -70 & -28 & 70 & -70 & -28\end{array}$

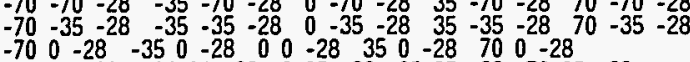

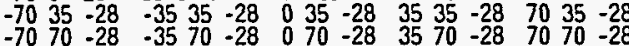

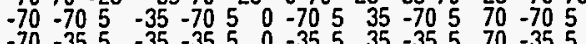

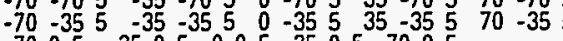

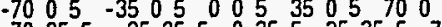

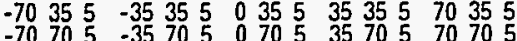

$\begin{array}{llllllllllllllllllllll}-70 & -70 & 38 & -35 & -70 & 38 & 0 & -70 & 38 & 35 & -70 & 38 & 70 & -70 & 38\end{array}$

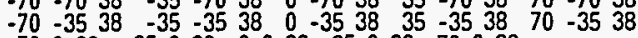

$\begin{array}{llllllllllllllll}-70 & 0 & 38 & -35 & 0 & 38 & 0 & 0 & 38 & 35 & 0 & 38 & 70 & 0 & 38 & \end{array}$

$\begin{array}{lllllllllllllllll}-70 & 35 & 38 & -35 & 35 & 38 & 0 & 35 & 38 & 35 & 35 & 38 & 70 & 35 & 38\end{array}$

$\begin{array}{lllllllllllllll}-70 & -70 & 71 & -35 & -30 & 71 & 0 & -70 & 71 & 35 & -70 & 71 & 70 & -70 & 71 \\ -70 & -35 & 71 & -35 & -35 & 71 & 0 & -35 & 71 & 35 & -35 & 71 & 70 & -35 & 71\end{array}$

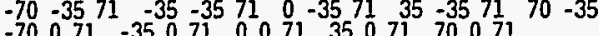

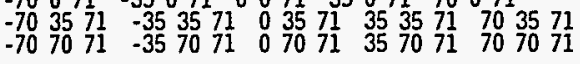

print 